\title{
Rotaxane-Branched Dendrimers with Enhanced Photosensitization
}

Wei-Jian $\mathrm{Li}^{\dagger}$, Zhubin $\mathrm{Hu}^{\S}$, Lin $\mathrm{Xu}^{\dagger, *}$, Xu-Qing Wang ${ }^{\dagger}$, Wei Wang ${ }^{\dagger}$, Guang-Qiang Yin ${ }^{\dagger, \#, ~ D a n-Y a n g ~}$ Zhang ${ }^{\dagger}$, Zhenrong Sun ${ }^{\S}$, Xiaopeng Li ${ }^{\#}$, Haitao Sun ${ }^{\S,}$, Hai-Bo Yang ${ }^{\dagger},{ }^{*}$

†Shanghai Key Laboratory of Green Chemistry and Chemical Processes \& Chang-Kung Chuang Institute, School of Chemistry and Molecular Engineering, East China Normal University, $3663 \mathrm{~N}$. Zhongshan Road, Shanghai 200062, P. R. China.

E-mail: 1xu@chem.ecnu.edu.cn (L. Xu); hbyang@chem.ecnu.edu.cn (H.-B. Yang)

${ }^{\S}$ State Key Laboratory of Precision Spectroscopy, School of Physics and Electronic Science, East China Normal University, 500 Dongchuan Road, Shanghai 200241, P. R. China.

E-mail: htsun@phy.ecnu.edu.cn

${ }^{\#}$ College of Chemistry and Environmental Engineering, Shenzhen University, Shenzhen, 518055, China. 


\section{Table of Contents}

Section A. Materials and general methods

Section B. Synthesis and characterization of the building block [2]rotaxane R-AN

Section C. Synthesis and characterization of the rotaxane-branched dendrimers

1. Synthesis of the rotaxane-branched dendrimers PG1-PG3

2. NMR and MS spectra of the rotaxane-branched dendrimers PG1-PG3

3. 2-D DOSY, GPC and DLS spectra of the rotaxane-branched dendrimers PG1-PG3

4. AFM images for the rotaxane-branched dendrimers PG1-PG3

Section D. Controllable generation of singlet oxygen

Section E. Mechanism study on photosensitization effect by TD-DFT

Section F. Photolysis of the rotaxane-branched dendrimers

Section G. Photooxidation mechanism of the rotaxane-branched dendrimers

Section H. Kinetic analysis on the photolysis of the rotaxane-branched dendrimers

Section I. Supplementary data

Section J. References 


\section{Section A. Materials and general methods}

All reagents were commercially available and used as supplied without further purification, compounds S1-S8 were prepared according to the published procedures. ${ }^{\text {S1- }}$ ${ }^{2}$ Deuterated solvents were purchased from Cambridge Isotope Laboratory (Andover, MA).

All solvents were dried according to standard procedures and all of them were degassed under $\mathrm{N}_{2}$ for 30 minutes before use. All air-sensitive reactions were carried out under inert $\mathrm{N}_{2}$ atmosphere. ${ }^{1} \mathrm{H}$ NMR, ${ }^{13} \mathrm{C}$ NMR and ${ }^{31} \mathrm{P}$ NMR spectra were recorded on Bruker $400 \mathrm{MHz}$ Spectrometer $\left({ }^{1} \mathrm{H}: 400 \mathrm{MHz} ;{ }^{13} \mathrm{C}: 101 \mathrm{MHz},{ }^{31} \mathrm{P}: 162 \mathrm{MHz}\right)$ and Bruker $500 \mathrm{MHz}$ Spectrometer $\left({ }^{1} \mathrm{H}: 500 \mathrm{MHz} ;{ }^{13} \mathrm{C}: 126 \mathrm{MHz},{ }^{31} \mathrm{P}: 202 \mathrm{MHz}\right)$ at $298 \mathrm{~K}$. The ${ }^{1} \mathrm{H}$ and ${ }^{13} \mathrm{C}$ NMR chemical shifts are reported relative to residual solvent signals, and ${ }^{31} \mathrm{P}\left\{{ }^{1} \mathrm{H}\right\}$ NMR chemical shifts are referenced to an external unlocked sample of $85 \% \mathrm{H}_{3} \mathrm{PO}_{4}(\delta 0.0)$. 2D NMR spectra $\left({ }^{1} \mathrm{H}-{ }^{1} \mathrm{H}\right.$ COSY, ROESY and DOSY) were recorded on Bruker $500 \mathrm{MHz}$ Spectrometer $\left({ }^{1} \mathrm{H}: 500 \mathrm{MHz}\right)$ at $298 \mathrm{~K}$.

DLS measurements were performed under a Malvern Zetasizer Nano-ZS light scattering apparatus (Malvern Instruments, U.K.) with a He-Ne laser (633 nm, $4 \mathrm{~mW})$. The MALDI MS experiments were carried out on a Shimadzu Axima Performance MALDI TOF/TOF mass spectrometer, equipped with a $337 \mathrm{~nm}$ nitrogen laser. The instrument was operated in positive ion reflectron mode and the accelerating voltage was $20 \mathrm{kV}$. DCTB matrix dissolved in THF at $20 \mathrm{mg} / \mathrm{mL}$ was used as matrix. The samples were dissolved in THF at $5-10 \mathrm{mg} / \mathrm{mL}$. Electrospray ionization (ESI) mass spectra were recorded with a Waters Synapt G2 mass spectrometer. All spectra were measured in positive reflectron or linear mode.

UV-vis spectra were recorded in a quartz cell (light path $10 \mathrm{~mm}$ ) on a Shimadzu UV2700 UV-visible spectrophotometer. Steady-state fluorescence spectra were recorded in a conventional quartz cell (light path $10 \mathrm{~mm}$ ) on a Shimadzu RF-6000 fluorescence spectrophotometer (slit width $5 \mathrm{~nm} / 5 \mathrm{~nm}$ for ex/em except for special instructions).

Fluorescence quantum yields were measured in absolutely in solution using a commercial fluorometer with integrating sphere (RF-6000, Shimadzu, slit width $5 \mathrm{~nm} / 5$ $\mathrm{nm}$ for ex/em).

The AFM samples were prepared by drop casting method using mica sheet as substrate. All the AFM images were obtained on a Dimension FastScan (Bruker), using ScanAsyst mode under ambient condition. General procedure of preparing AFM samples: the solution of rotaxane-branched dendrimers PG1-PG3 in THF $\left(c=10^{-7} \mathrm{M}\right)$ were prepared, the samples were prepared by drop casting method using mica sheet as substrate. 
Section B. Synthesis and characterization of the building block [2]rotaxane $R-A N$

Scheme S1. The synthesis route of the macrocycle $\mathbf{A N}{ }^{\mathrm{S} 1}$.

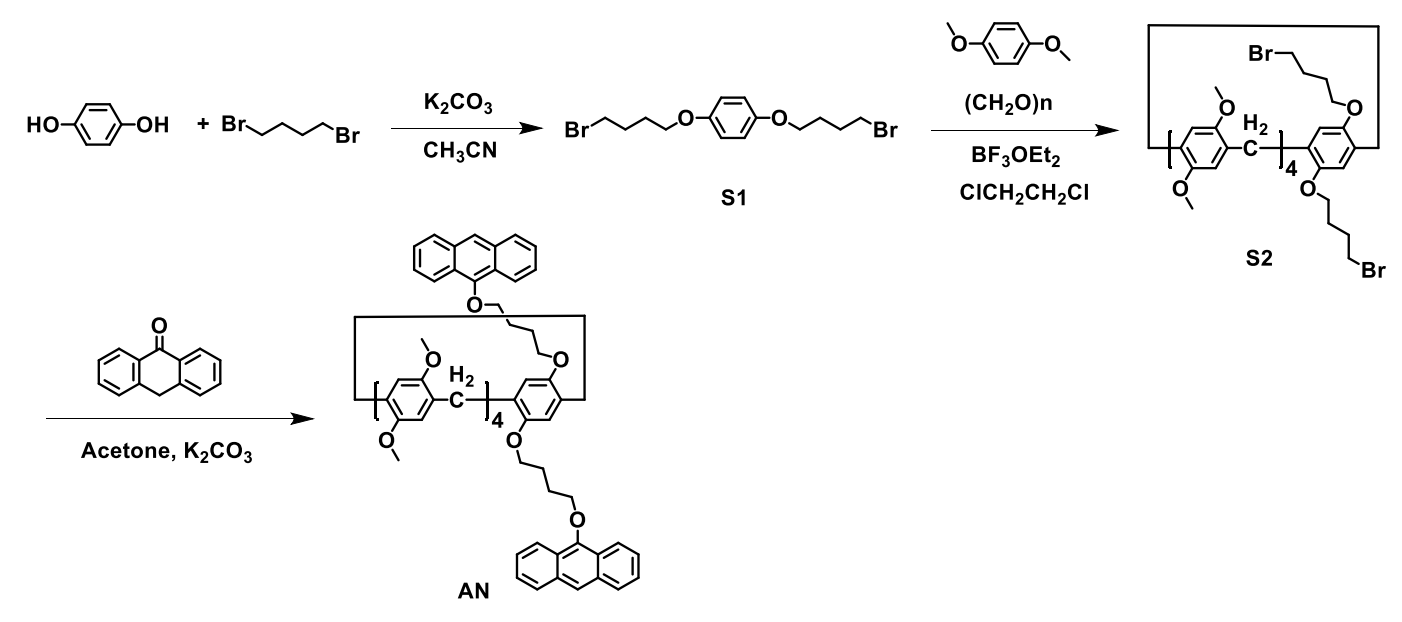

Scheme S2. The synthesis route of [2] rotaxane R-AN ${ }^{\text {S2 }}$.
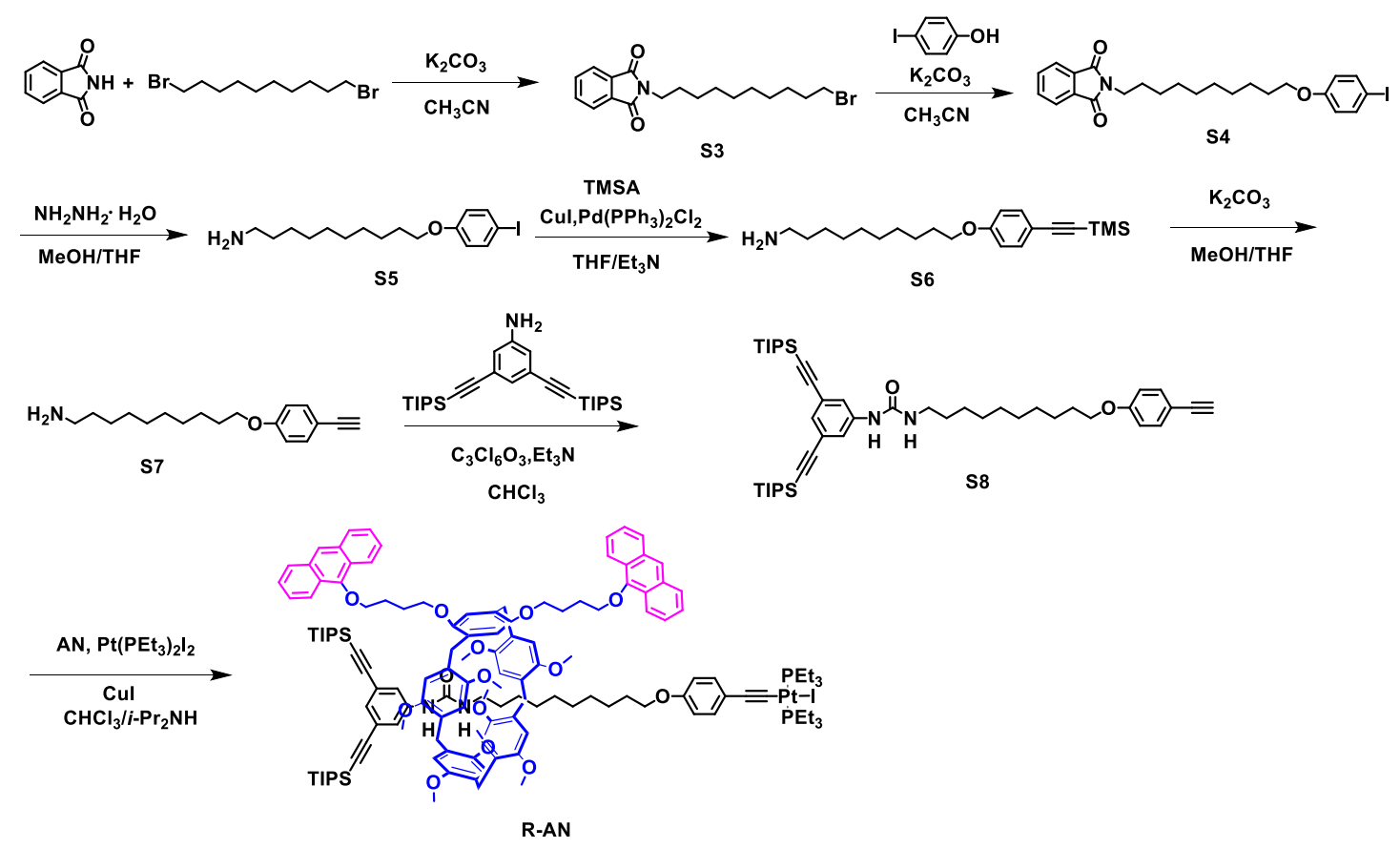

Synthesis of AN: Mixing compound S2 (3 g, $3.02 \mathrm{mmol}$ ) and anthranone (2.35 g, 12.09 mmol) in acetone (pre-dried by $\mathrm{Na}_{2} \mathrm{SO}_{4}, 100 \mathrm{~mL}$ ), then $\mathrm{K}_{2} \mathrm{CO}_{3}(4.17 \mathrm{~g}, 30.22 \mathrm{mmol}$ ) was added into the reaction flask. The resultant suspension was refluxed overnight. After cooling to room temperature, the reaction mixture was filtered and the filtrate was concentrated in vacuum. The resultant residue was purified by column chromatography $\left(\mathrm{SiO}_{2} ; \mathrm{PE} / \mathrm{DCM}\right)$ to yield a pale yellow solid AN (1.78g, 48\%). ${ }^{1} \mathrm{H}$ NMR (400 MHz, $\left.\mathrm{CD}_{2} \mathrm{Cl}_{2}\right): \delta 8.18-8.24(\mathrm{~m}, 6 \mathrm{H}), 7.94-7.96(\mathrm{~m}, 4 \mathrm{H}), 7.40-7.42(\mathrm{~m}, 8 \mathrm{H}), 6.76-6.89(\mathrm{~m}$, $10 \mathrm{H}), 4.20-4.22(\mathrm{~m}, 4 \mathrm{H}), 3.97-4.02(\mathrm{~m}, 4 \mathrm{H}), 3.61-3.78(\mathrm{~m}, 34 \mathrm{H}), 2.20-2.26(\mathrm{~m}, 8 \mathrm{H})$. ${ }^{13} \mathrm{C}$ NMR (126 MHz, $\left.\mathrm{CD}_{2} \mathrm{Cl}_{2}\right): \delta 152.91,151.92,151.91,151.85,151.26,134.07$, 
$130.02,129.94,129.80,129.77,129.75,129.67,127.10,126.76,126.26,123.81$, $123.58,115.79,114.85,114.84,114.82,114.73$, 77.39, 69.62, 57.07, 56.98, 56.97, 56.94, 30.74, 30.45, 29.30, 28.52. HRMS (MALDI-TOF-MS): Calculated for [AN + $\mathrm{Na}]^{+}$: 1241.5391; Found: 1241.5389; Calculated for $[\mathbf{A N}+\mathrm{K}]^{+}:$1257.5130; Found: 1257.5153 .

Synthesis of [2]rotaxane R-AN: A Schlenk flask was charged with macrocycle component AN (1.62 g, $1.33 \mathrm{mmol})$, thread component $\mathbf{S 8}$ (200 mg, $0.27 \mathrm{mmol}$ ) and $\mathrm{Pt}\left(\mathrm{PEt}_{3}\right)_{2} \mathrm{I}_{2}(728 \mathrm{mg}, 1.06 \mathrm{mmol})$. The Schlenk flask was then evacuated and back-filled with $\mathrm{N}_{2}$ three times. Next, the mixture solvent of degassed $\mathrm{CHCl}_{3}$ and $i-\mathrm{Pr}_{2} \mathrm{NH}(\mathrm{v} / \mathrm{v}, 8 / 4$ $\mathrm{mL}$ ) was added via syringe. The resultant solution was stirred for $2 \mathrm{~h}$ under $-10^{\circ} \mathrm{C}$. Then a catalytic amount of $\mathrm{CuI}$ was added to the mixture under an inert atmosphere. The reaction mixture was allowed to warm to room temperature and stirred overnight. The solution was concentrated and the residue was purified by column chromatography $\left(\mathrm{SiO}_{2} ; \mathrm{PE} / \mathrm{DCM}\right)$ and preparative gel permeation chromatography (GPC). A pale yellow solid R-AN (566 mg, 84\%) was obtained. ${ }^{1} \mathrm{H}$ NMR (500 MHz, $\mathrm{CD}_{2} \mathrm{Cl}_{2}$ ): $\delta$ 8.22-8.24 (m, 2H), 8.13-8.17 (m, 4H), 7.90-7.95 (m, 4H), 7.53 (s, 2H), 7.35-7.41 (m, 8H), 7.08$7.11(\mathrm{~m}, 3 \mathrm{H}), 6.88-6.97(\mathrm{~m}, 7 \mathrm{H}), 6.77-6.79(\mathrm{~m}, 4 \mathrm{H}), 6.62-6.64(\mathrm{~d}, J=10.0 \mathrm{~Hz}, 2 \mathrm{H})$, $4.22-4.25$ (t, $J=7.5 \mathrm{~Hz}, 2 \mathrm{H}), 4.11-4.14(\mathrm{t}, J=7.5 \mathrm{~Hz}, 2 \mathrm{H}), 4.07(\mathrm{~m}, 2 \mathrm{H}), 3.89(\mathrm{~m}, 2 \mathrm{H})$, 3.64-3.79 (m, 36H), $2.41(\mathrm{~m}, 1 \mathrm{H}), 2.09-2.14(\mathrm{~m}, 20 \mathrm{H}), 1.54(\mathrm{~m}, 2 \mathrm{H}), 1.00-1.18(\mathrm{~m}$, $66 \mathrm{H}), 0.70(\mathrm{~m}, 2 \mathrm{H}), 0.55(\mathrm{~m}, 2 \mathrm{H}), 0(\mathrm{~m}, 2 \mathrm{H}),-1.45(\mathrm{~m}, 2 \mathrm{H}),-1.97(\mathrm{~m}, 2 \mathrm{H}) .{ }^{31} \mathrm{P}$ NMR (202 MHz, $\left.\mathrm{CD}_{2} \mathrm{Cl}_{2}\right): \delta 8.77 .{ }^{13} \mathrm{C} \mathrm{NMR}\left(126 \mathrm{MHz}, \mathrm{CD}_{2} \mathrm{Cl}_{2}\right): \delta 158.79,154.84,152.94$, $152.86,152.58,152.22,152.12,152.09,151.98,151.96,151.90,151.81,151.50$, $151.48,142.39,134.07,134.04,133.22,130.86,130.56,130.35,130.15,130.08$, $130.04,130.00,129.89,129.77,127.11,127.02,126.80,126.70,126.28,126.27$, $125.55,123.92,123.76,123.63,123.44,123.31,122.41,116.99,116.35,116.04$, $115.78,115.75,115.71,115.65,114.75,114.70,114.65,107.94,100.98,92.40,88.48$, $88.36,88.25,77.39,77.30,70.30,69.64,69.51,61.80,58.72,57.96,57.77,57.72,56.97$, $56.90,56.88,32.54,32.11,31.66,31.60,31.28,31.01,30.80,30.71,30.65,30.61,30.52$, 29.39, 29.10, 28.71, 28.48, 28.36, 27.86, 26.89, 20.03, 19.97, 18.31, 18.17, 18.03, 12.85, 9.64. HRMS (ESI-TOF-MS): Calculated for [R-AN $+\mathrm{H}]^{+}:$2533.1433; Found: 2532.9878. Calculated for [R-AN $+2 \mathrm{H}]^{2+}:$ 1265.0560; Found: 1265.1927. 


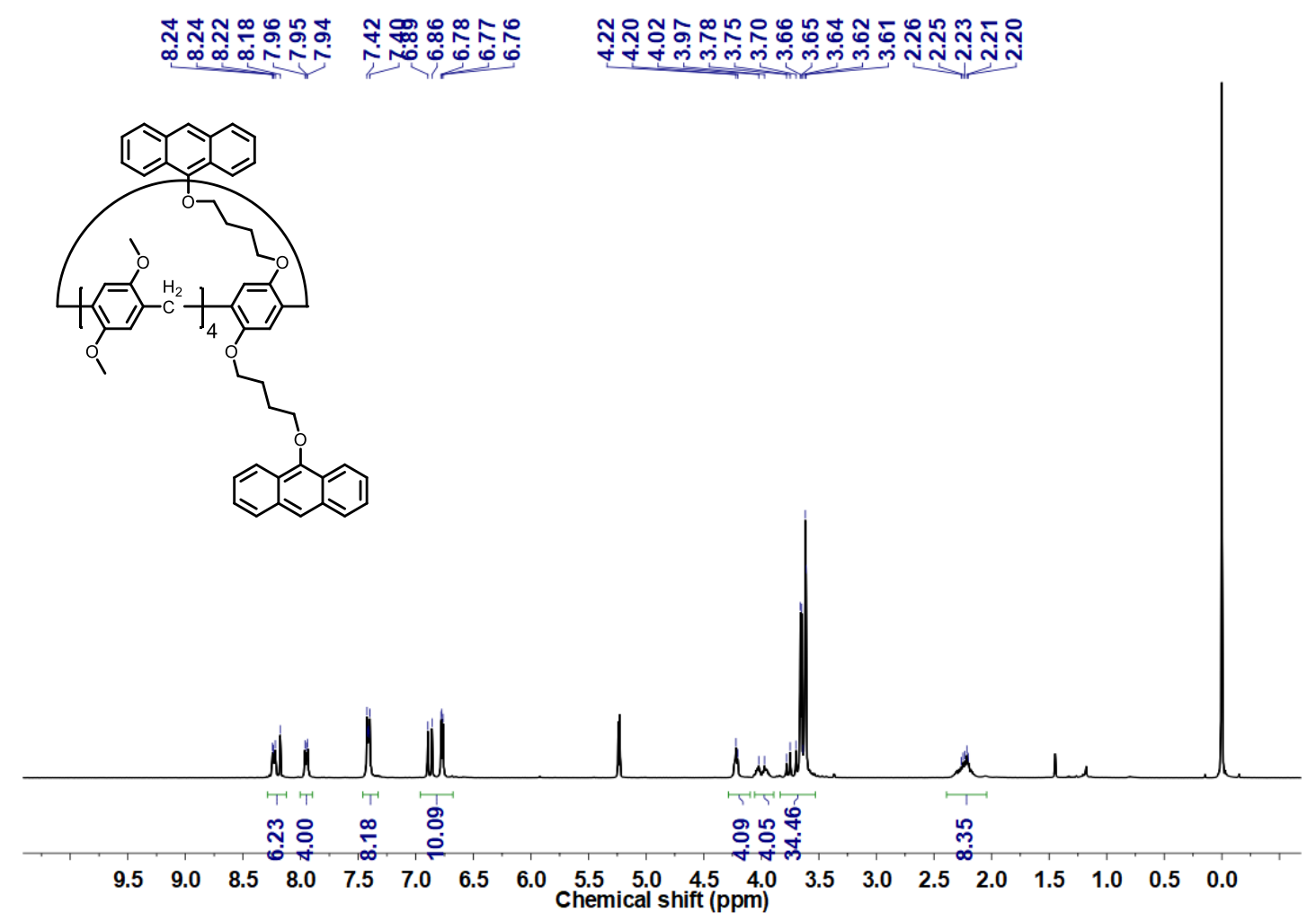

Figure S1. ${ }^{1} \mathrm{H}$ NMR spectrum $\left(\mathrm{CD}_{2} \mathrm{Cl}_{2}, 298 \mathrm{~K}, 400 \mathrm{MHz}\right)$ of the macrocycle $\mathbf{A N}$.

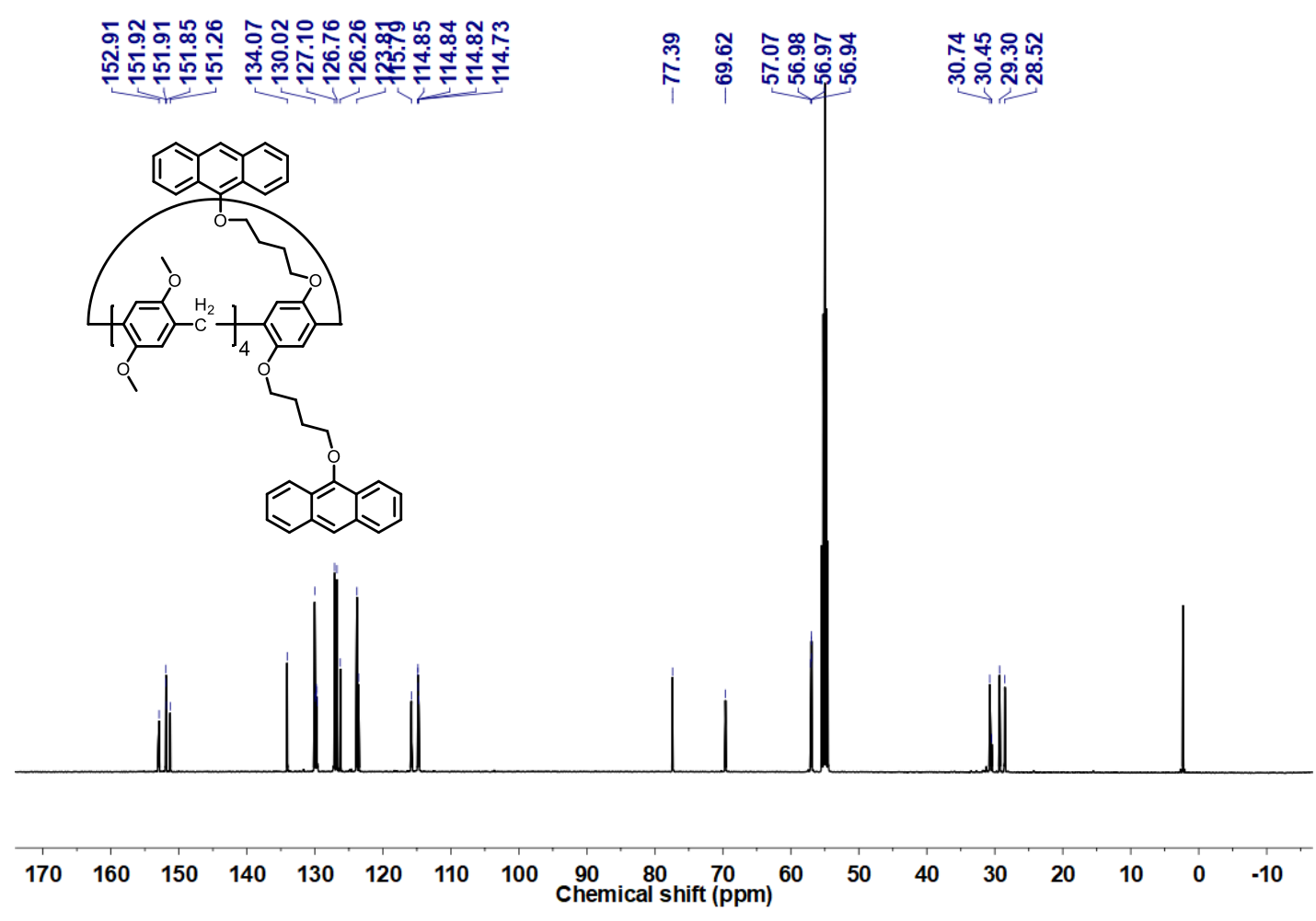

Figure S2. ${ }^{13} \mathrm{C}$ NMR spectrum $\left(\mathrm{CD}_{2} \mathrm{Cl}_{2}, 298 \mathrm{~K}, 126 \mathrm{MHz}\right)$ of the macrocycle $\mathbf{A N}$. 


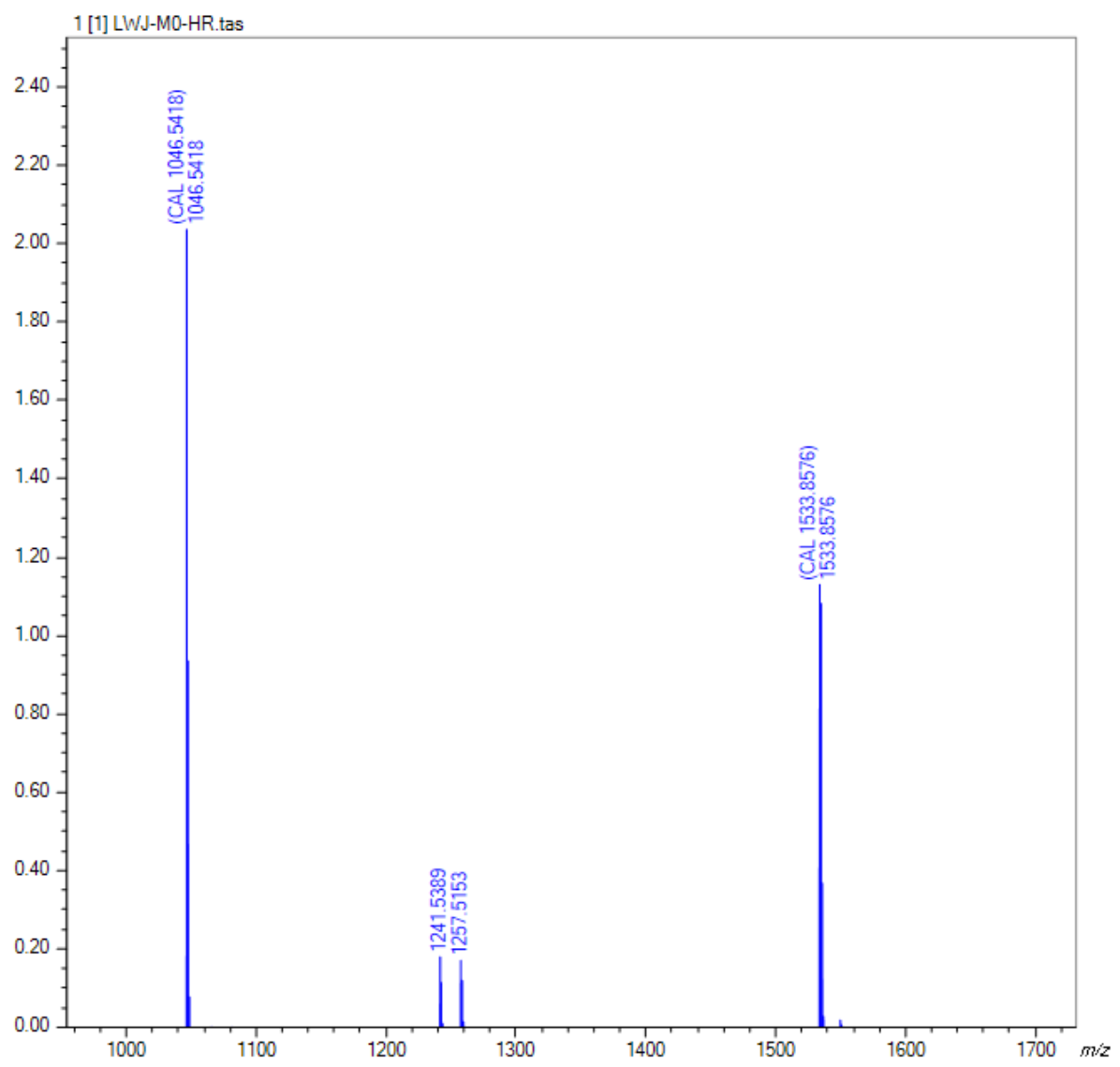

Figure S3. HRMS (MALDI-TOF-MS) spectrum of the macrocycle AN.

(a)

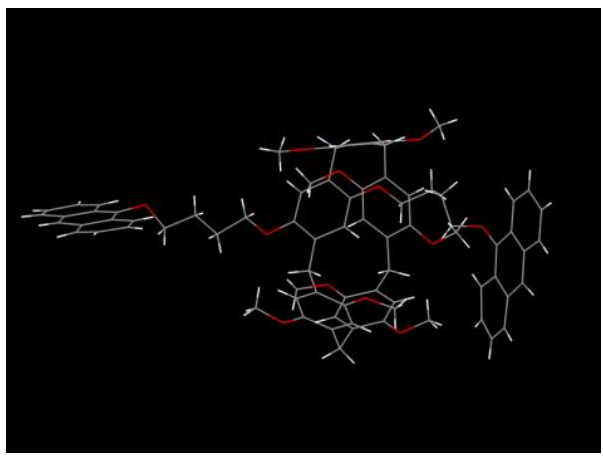

(b)

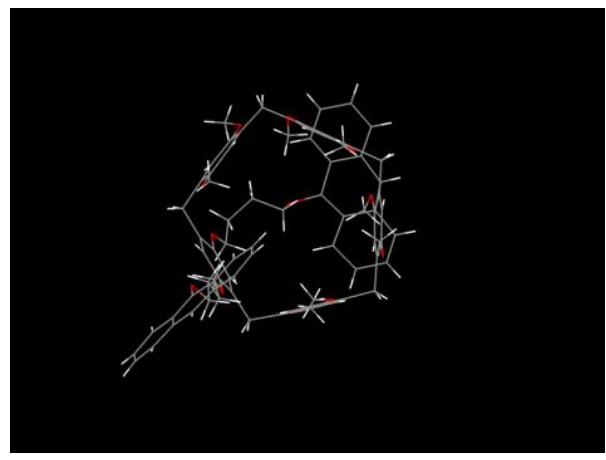

Figure S4. (a) Side view and (b) front view of the crystal structure AN, C gray, O red, $\mathrm{H}$ white. The X-ray crystallographic coordinates for structure have been deposited at the Cambridge Crystallographic Data Centre (CCDC), under deposition numbers CCDC 1999903. These data can be obtained free of charge from The Cambridge Crystallographic Data Centre via www.ccdc.cam.ac.uk/data_request/cif. 
Table S1. Crystallographic data and structure refinement for the macrocycle AN.

\begin{tabular}{|c|c|}
\hline Identification code & exp_859 \\
\hline Empirical formula & $\mathrm{C}_{141} \mathrm{H}_{142} \mathrm{O}_{23}$ \\
\hline Formula weight & 2204.54 \\
\hline Temperature/K & $100.00(10)$ \\
\hline Crystal system & triclinic \\
\hline Space system & $\mathrm{P}-1$ \\
\hline Unit cell dimensions & $\begin{array}{ll}\mathrm{a}=11.7550(3) \AA & \alpha=75.495(2)^{\circ} \\
\mathrm{b}=24.7652(5) \AA & \beta=80.685(2)^{\circ} \\
\mathrm{c}=27.7101(5) \AA & \gamma=77.163(2)^{\circ}\end{array}$ \\
\hline Volume $/ \AA^{3}$ & $7566.1(3)$ \\
\hline Z & 2 \\
\hline$\rho_{\text {calc }} / \mathrm{g} \mathrm{cm}^{-3}$ & 0.968 \\
\hline$\mu / \mathrm{mm}^{-1}$ & 0.522 \\
\hline $\mathrm{F}(000)$ & 2344.0 \\
\hline Crystal size $/ \mathrm{mm}^{3}$ & $0.26 \times 0.14 \times 0.08$ \\
\hline Radiation & $\operatorname{CuK} \alpha(\lambda=1.54184)$ \\
\hline $2 \Theta$ range for data collection $/^{\circ}$ & 7.512 to 155.474 \\
\hline Index ranges & $-14 \leq h \leq 13,-30 \leq k \leq 30,-34 \leq l \leq 34$ \\
\hline Reflections collected & 208558 \\
\hline Independent reflections & $30262\left[\mathrm{R}_{\text {int }}=0.1516, \mathrm{R}_{\text {sigma }}=0.1287\right]$ \\
\hline Data/restraints/parameters & $30262 / 247 / 1483$ \\
\hline Goodness-of-fit on $F^{2}$ & 0.983 \\
\hline Final $\mathrm{R}$ indexes $[I>=2 \sigma(I)]$ & $\mathrm{R}_{1}=0.1121, \mathrm{wR}_{2}=0.3051$ \\
\hline Final $\mathrm{R}$ indexes [all data] & $\mathrm{R}_{1}=0.1581, \mathrm{wR}_{2}=0.3391$ \\
\hline Largest diff. peak/hole / $e \AA^{-3}$ & $2.04 /-0.67$ \\
\hline
\end{tabular}




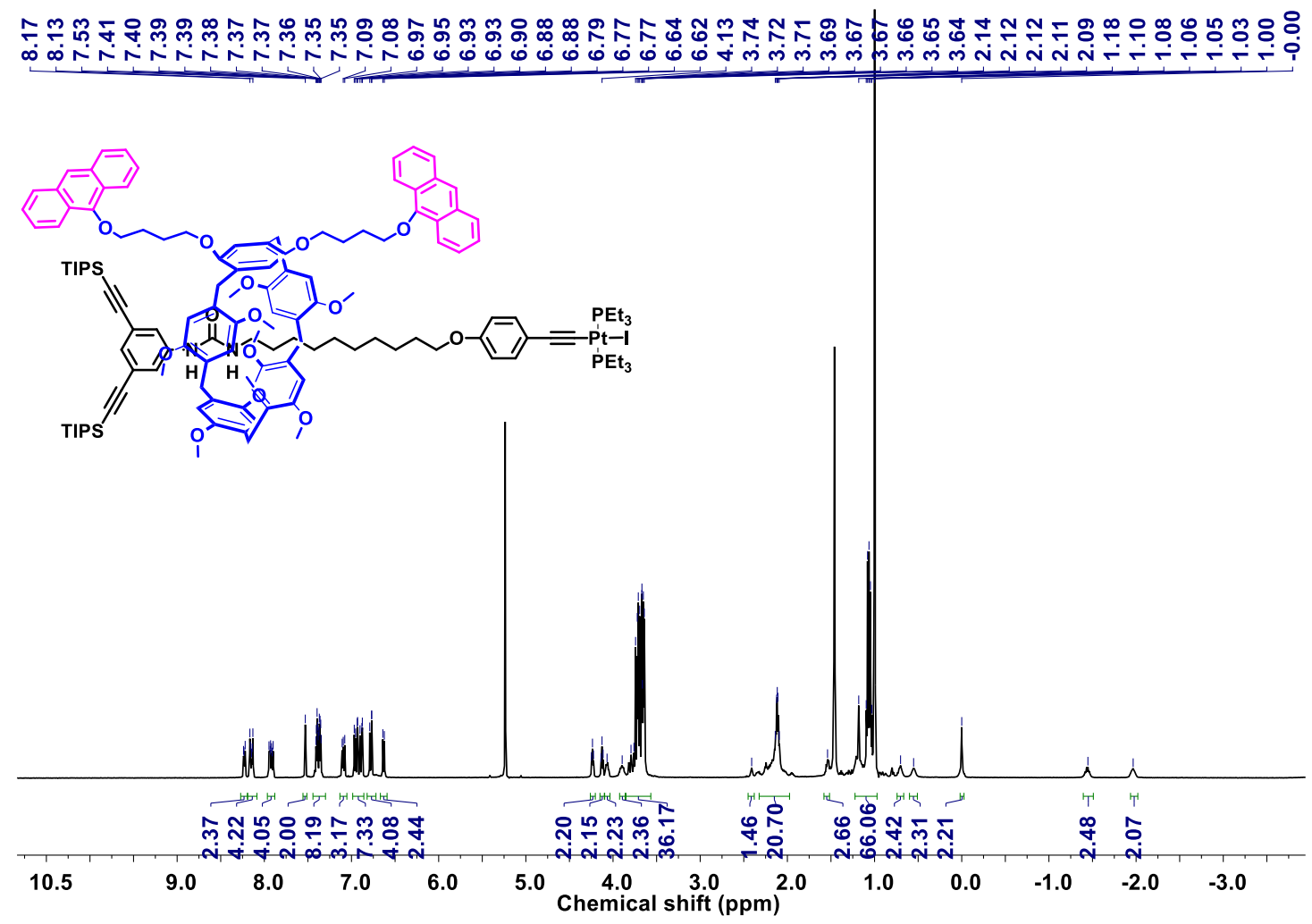

Figure S5. ${ }^{1} \mathrm{H}$ NMR spectrum $\left(\mathrm{CD}_{2} \mathrm{Cl}_{2}, 298 \mathrm{~K}, 500 \mathrm{MHz}\right)$ of [2] rotaxane R-AN.

$$
\underset{\substack{\hat{\infty} \\ 1}}{\substack{0 \\ i}}
$$
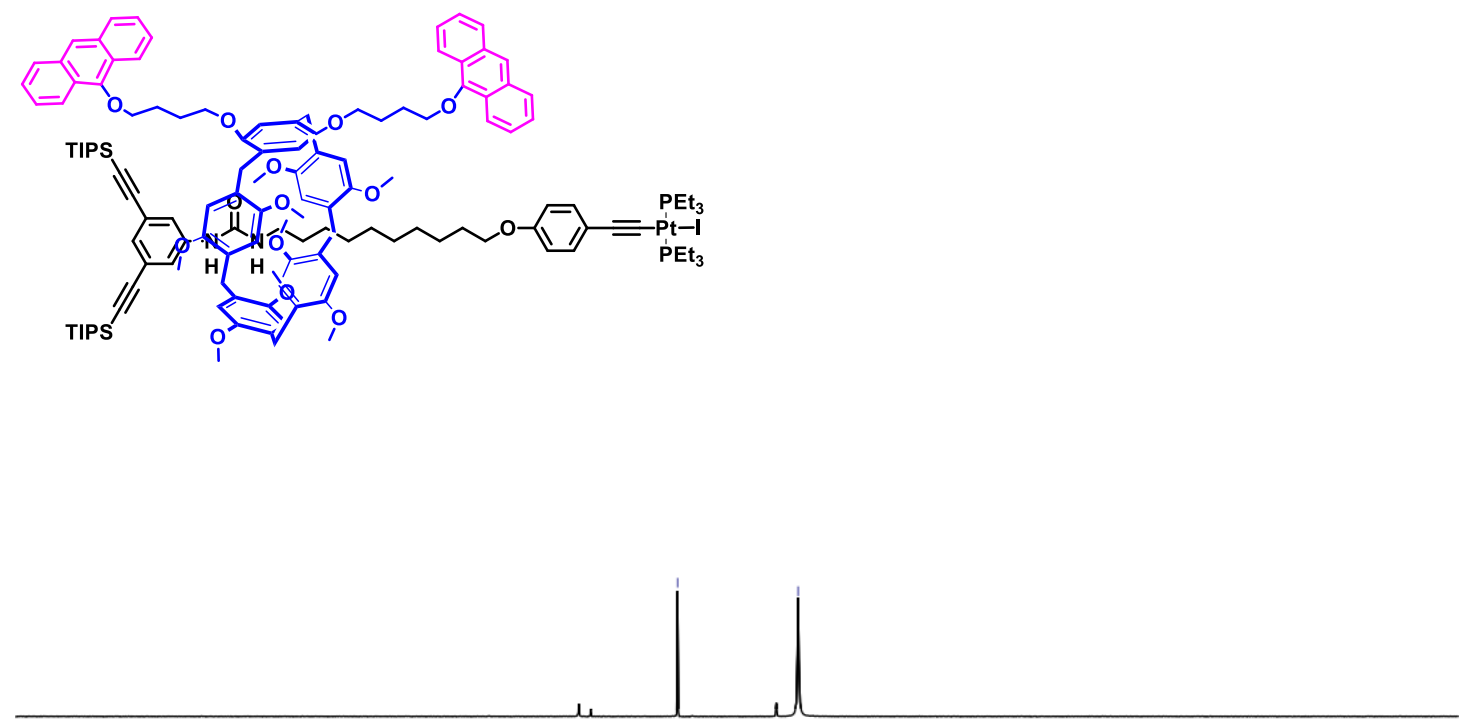

Figure S6. ${ }^{31} \mathrm{P}$ NMR spectrum $\left(\mathrm{CD}_{2} \mathrm{Cl}_{2}, 298 \mathrm{~K}, 202 \mathrm{MHz}\right)$ of [2] rotaxane R-AN. 


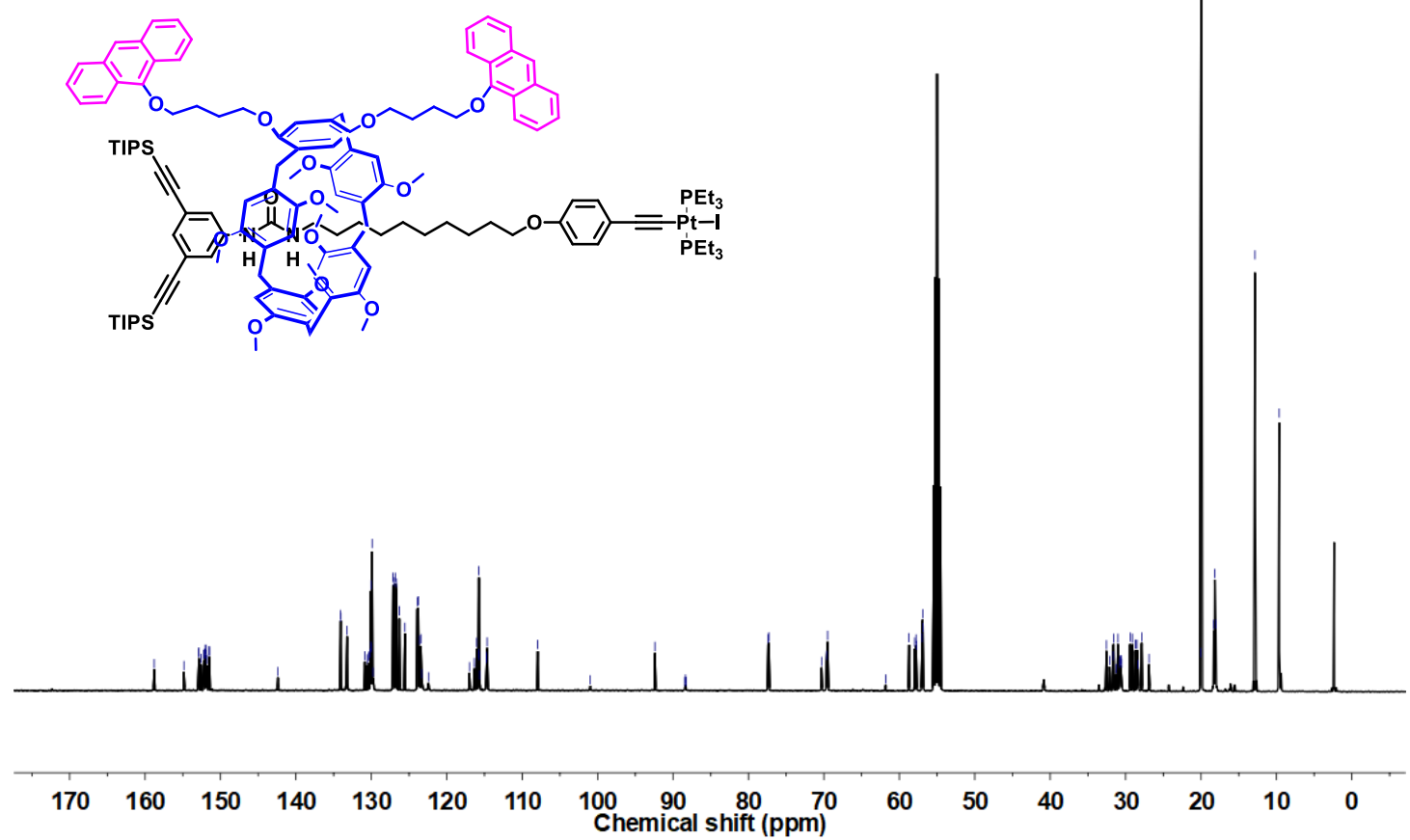

Figure S7. ${ }^{13} \mathrm{C}$ NMR spectrum $\left(\mathrm{CD}_{2} \mathrm{Cl}_{2}, 298 \mathrm{~K}, 126 \mathrm{MHz}\right)$ of [2]rotaxane R-AN.

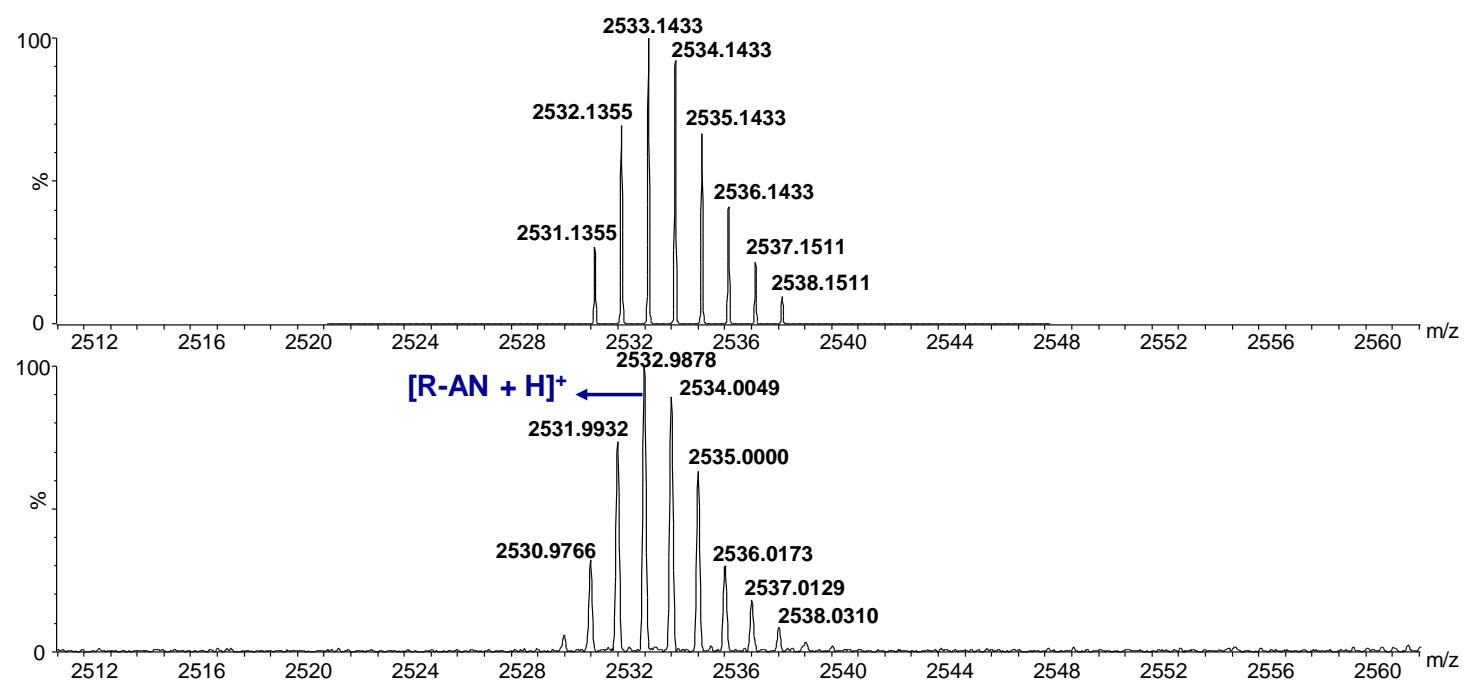

Figure S8. Theoretical (top) and experimental (bottom) ESI-TOF-MS spectra of [2]rotaxane R-AN. 


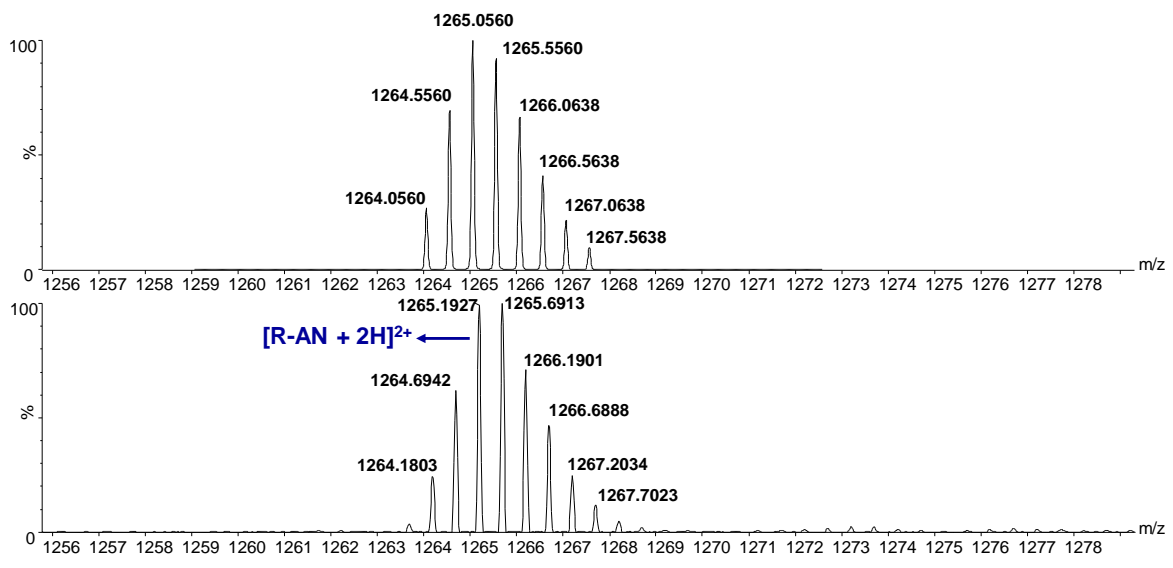

Figure S9. Theoretical (top) and experimental (bottom) ESI-TOF-MS spectra of [2]rotaxane R-AN.

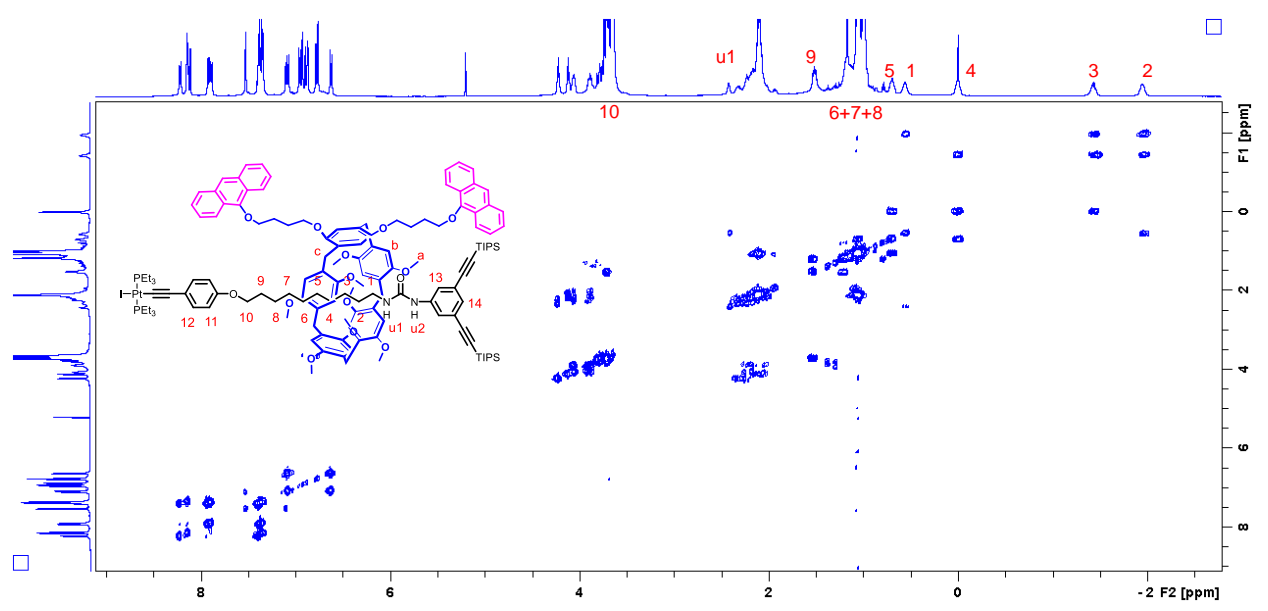

Figure S10. 2D ${ }^{1} \mathrm{H}-{ }^{1} \mathrm{H}$ COSY spectrum $\left(\mathrm{CD}_{2} \mathrm{Cl}_{2}, 298 \mathrm{~K}, 500 \mathrm{MHz}\right)$ of [2] rotaxane RAN.
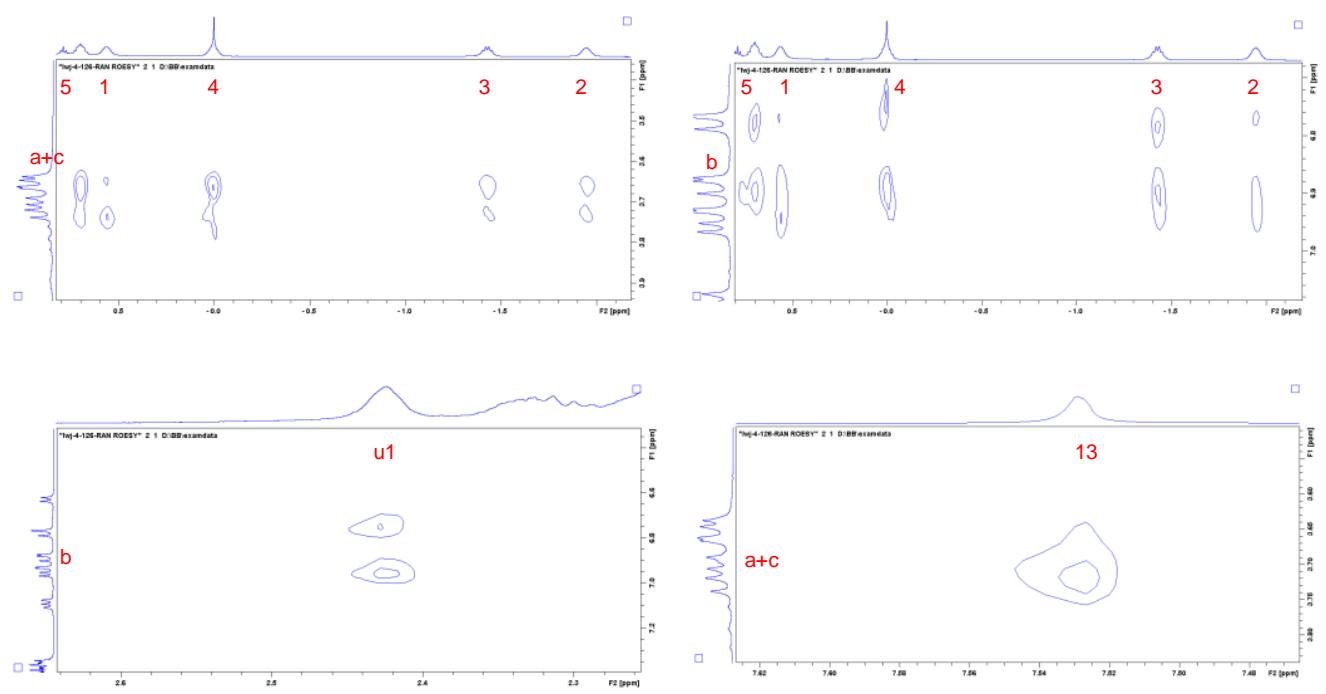

Figure S11. 2D ${ }^{1} \mathrm{H}-{ }^{1} \mathrm{H}$ ROESY spectrum $\left(\mathrm{CD}_{2} \mathrm{Cl}_{2}, 298 \mathrm{~K}, 500 \mathrm{MHz}\right)$ of [2] rotaxane R-AN. 


\section{Section C. Synthesis and characterization of the rotaxane-branched dendrimers}

1. Synthesis of the first-generation rotaxane-branched dendrimer PG1.

Scheme S3. Synthesis route of the rotaxane-branched dendrimer PG1.

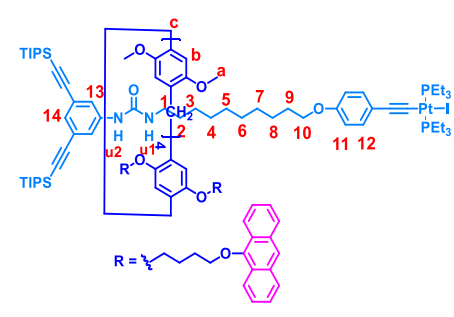

[2]rotaxane R-AN
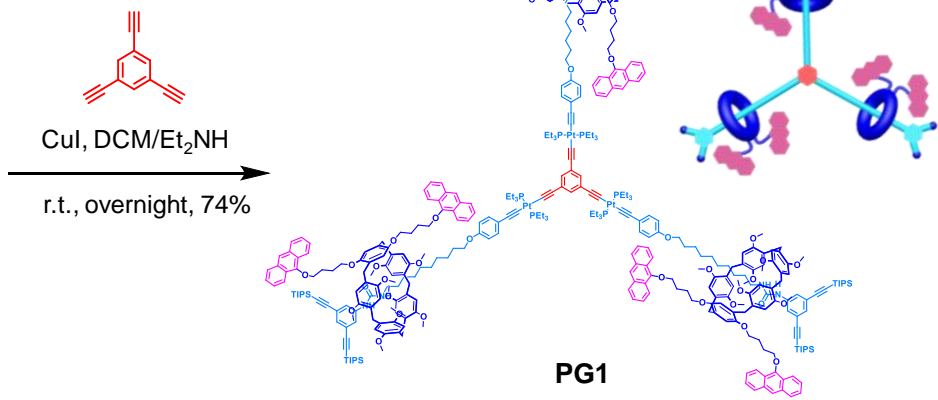

Synthesis of the first-generation rotaxane-branched dendrimer PG1: A mixture of 1, 3, 5-triethynylbenzene (5.4 mg, $0.036 \mathrm{mmol}$ ) and [2] rotaxane R-AN (300 mg, 0.119 mmol) were added in a Schlenk flask, the Schlenk flask was then evacuated and backfilled with $\mathrm{N}_{2}$ three times. Next, the degassed $\mathrm{Et}_{2} \mathrm{NH}$ and $\mathrm{CH}_{2} \mathrm{Cl}_{2}(\mathrm{v} / \mathrm{v}, 10 / 10 \mathrm{~mL})$ and a catalytic amount of $\mathrm{CuI}$ was added under an inert atmosphere. The reaction was stirred overnight at room temperature. The solvent was evaporated and the residue was purified by column chromatography $\left(\mathrm{SiO}_{2} ; \mathrm{PE} / \mathrm{DCM}\right)$ and preparative gel permeation chromatography (GPC) to yield a pale yellow solid PG1 (194 mg, 74\%). ${ }^{1} \mathrm{H}$ NMR (500 $\left.\mathrm{MHz}, \mathrm{CD}_{2} \mathrm{Cl}_{2}\right): \delta 8.31-8.33(\mathrm{~m}, 6 \mathrm{H}), 8.22-8.26(\mathrm{~m}, 12 \mathrm{H}), 7.99-8.04(\mathrm{~m}, 12 \mathrm{H}), 7.63(\mathrm{~m}$, $6 \mathrm{H}), 7.44-7.50(\mathrm{~m}, 24 \mathrm{H}), 7.20(\mathrm{~m}, 9 \mathrm{H}), 6.86-7.06(\mathrm{~m}, 36 \mathrm{H}), 6.71-6.73(\mathrm{~d}, J=10.0 \mathrm{~Hz}$, $6 \mathrm{H})$, 4.32-4.34 (m, 6H), 4.21-4.23 (m, 6H), 4.16-4.17 (m, 6H), 3.97-4.04 (m, 6H), 3.73$3.84(\mathrm{~m}, 108 \mathrm{H}), 2.50(\mathrm{~m}, 3 \mathrm{H}), 2.12-2.34(\mathrm{~m}, 60 \mathrm{H}), 1.63(\mathrm{~m}, 6 \mathrm{H}), 1.09-1.26(\mathrm{~m}, 198 \mathrm{H})$, $0.80(\mathrm{~m}, 6 \mathrm{H}), 0.64(\mathrm{~m}, 6 \mathrm{H}), 0.09(\mathrm{~m}, 6 \mathrm{H}),-1.35(\mathrm{~m}, 6 \mathrm{H}),-1.87(\mathrm{~m}, 6 \mathrm{H}) .{ }^{31} \mathrm{P}$ NMR $(202$ $\left.\mathrm{MHz}, \mathrm{CD}_{2} \mathrm{Cl}_{2}\right): \delta 11.65 .{ }^{13} \mathrm{C} \mathrm{NMR}\left(126 \mathrm{MHz}, \mathrm{CD}_{2} \mathrm{Cl}_{2}\right): \delta 154.81,152.93,152.86$, $152.58,152.22,152.11,152.08,151.96,151.94,151.88,151.79,151.49,151.46$, $142.38,134.06,134.03,133.38,130.85,130.55,130.34,130.14,130.03,129.98$, $129.87,129.74,127.11,127.01,126.79,126.69,126.27,126.26,125.53,123.91,123.76$, $123.61,123.42,116.99,116.33,116.03,115.77,115.71,115.65,114.73,114.69,114.63$, $107.93,92.39,77.38,77.30,70.29,69.64,69.50,58.72,57.96,57.77,57.71,56.96$, $56.90,56.88,40.84,32.54,32.10,31.61,31.26,31.02,30.78,30.69,30.63,30.59,30.50$, 29.38, 29.08, 28.69, 28.46, 28.32, 27.88, 26.86, 19.95, 18.09, 17.95, 17.81, 13.06, 12.83, 12.61, 9.72. LRMS (MALDI-TOF-MS): Calculated for $[\mathbf{P G 1}+\mathrm{H}]^{+}$: 7357.6; Found: 7360.3 . 


\section{Synthesis of the second-generation rotaxane-branched dendrimer PG2.}

Scheme S4. Synthesis route of the rotaxane-branched dendrimer PG2.

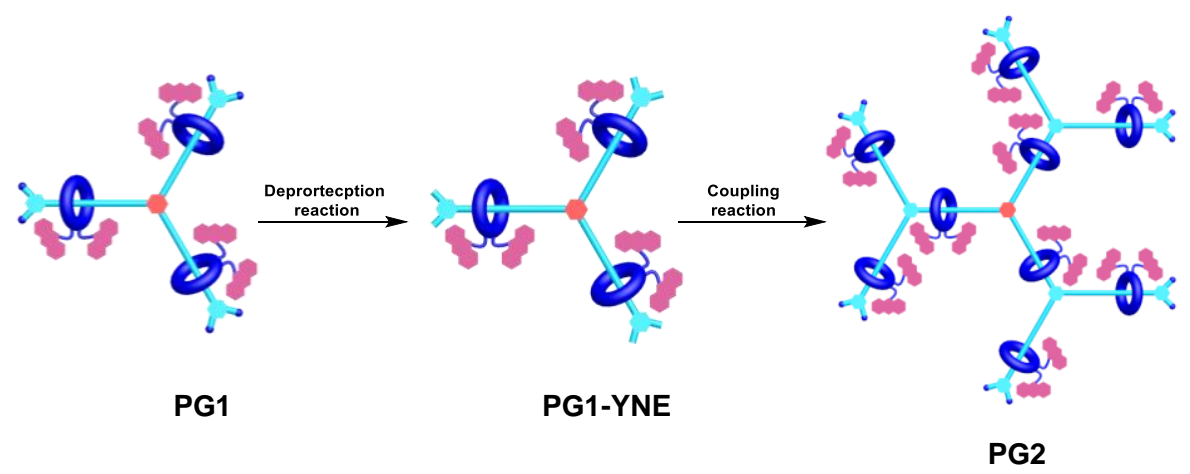

Synthesis of the rotaxane-branched dendrimer PG2: A reaction flask was charged with a THF solution of PG1 (100 mg, $0.0136 \mathrm{mmol}$ ) and then a solution of $\mathrm{Bu} 4 \mathrm{NF} \cdot 3 \mathrm{H}_{2} \mathrm{O}(52 \mathrm{mg}, 0.165 \mathrm{mmol})$ in THF was added dropwise into the reaction flask. The reaction mixture was stirred at room temperature for $2 \mathrm{~h}$, the obtained residue was washed by water, then dried with $\mathrm{Na}_{2} \mathrm{SO}_{4}$ and concentrated. The residue was further purified by column chromatography $\left(\mathrm{SiO}_{2} ; \mathrm{DCM}\right)$ and preparative gel permeation chromatography (GPC) to afford a pale-yellow solid PG1-YNE. Then the obtained PG1-YNE (54 mg, $0.0084 \mathrm{mmol}$ ) and [2]rotaxane R-AN (140 mg, $0.055 \mathrm{mmol}$ ) were added in a Schlenk flask, the Schlenk flask was then evacuated and back-filled with $\mathrm{N}_{2}$ three times. Next, the degassed $\mathrm{Et}_{2} \mathrm{NH}$ and $\mathrm{CH}_{2} \mathrm{Cl}_{2}(\mathrm{v} / \mathrm{v}, 10 / 10 \mathrm{~mL})$ and a catalytic amount of $\mathrm{CuI}$ were added under an inert atmosphere. The reaction was stirred overnight at room temperature. The solvent was evaporated and the residue was purified by column chromatography $\left(\mathrm{SiO}_{2} ; \mathrm{DCM} / \mathrm{EA}\right)$ and preparative gel permeation chromatography (GPC) to yield a pale yellow solid PG2 (112 mg, 69\%). ${ }^{1} \mathrm{H}$ NMR (500 $\left.\mathrm{MHz}, \mathrm{CD}_{2} \mathrm{Cl}_{2}\right): \delta 8.30-8.32(\mathrm{~m}), 8.21-8.26(\mathrm{~m}), 7.98-8.03(\mathrm{~m}), 7.61(\mathrm{~m}), 7.43-7.50(\mathrm{~m})$, 7.14-7.19 (m), 6.85-7.07 (m), 6.69-6.71(m), 4.31-4.33 (m), 4.22-4.22 (m), 4.15-4.16 (m), 3.98-4.00 (m), 3.72-3.86 (m), $2.90(\mathrm{~m}), 2.50(\mathrm{~m}), 2.10-2.42(\mathrm{~m}),, 1.62(\mathrm{~m}), 1.08-$ $1.23(\mathrm{~m}),, 0.78(\mathrm{~m}), 0.64(\mathrm{~m}),-0.08(\mathrm{~m}),-1.37(\mathrm{~m}),-1.54(\mathrm{~m})-1.80(\mathrm{~m}),-1.88(\mathrm{~m}) .{ }^{31} \mathrm{P}$ NMR (202 MHz, $\left.\mathrm{CD}_{2} \mathrm{Cl}_{2}\right): \delta 11.66,11.58 .{ }^{13} \mathrm{C} \mathrm{NMR} \mathrm{(126} \mathrm{MHz,} \mathrm{CD}_{2} \mathrm{Cl}_{2}$ ): $\delta$ 155.67, $154.85,152.97,152.92,152.85,152.58,152.54,152.22,152.12,152.09,151.97$, $151.95,151.90,151.80,151.55,151.50,151.47,142.37,134.07,134.03,133.52$, $130.85,130.66,130.56,130.51,130.35,130.25,130.16,130.05,130.00,129.88,127.11$, $127.02,126.87,126.80,126.70,126.27,126.26,125.54,123.95,123.91,123.76$, $123.64,123.43,116.98,116.35,116.04,115.74,115.58,114.75,114.71,114.65,107.93$, $92.40,77.42,77.39,77.30,70.30,70.18,69.65,69.55,58.71,58.64,57.96,57.77,57.71$, $56.97,56.90,56.88,41.41,40.86,33.51,32.80,32.63,32.54,32.10,32.00,31.66,31.60$, $31.27,30.98,30.79,30.70,30.65,30.60,30.52,29.38,29.19,29.09,28.71,28.47,28.36$, 27.84, 27.76, 26.89, 26.65, 24.27, 20.08, 19.96, 19.83, 18.21, 18.07, 17.93, 15.47, 13.06, $12.95,12.90,12.84,12.79,12.70,12.61,9.78,9.64$. LRMS (MALDI-TOF-MS): Calculated for $[\mathbf{P G 2}+\mathrm{K}]^{+}:$20854.1; Found: 20856.7; Calculated for $[\mathbf{P G 2}+\mathrm{K}+\mathrm{Na}]^{2+}$ : 
10438.5; Found: 10437.6;

3. Synthesis of the third-generation rotaxane-branched dendrimer PG3.

Scheme S5. Synthesis route of the rotaxane-branched dendrimer PG3.
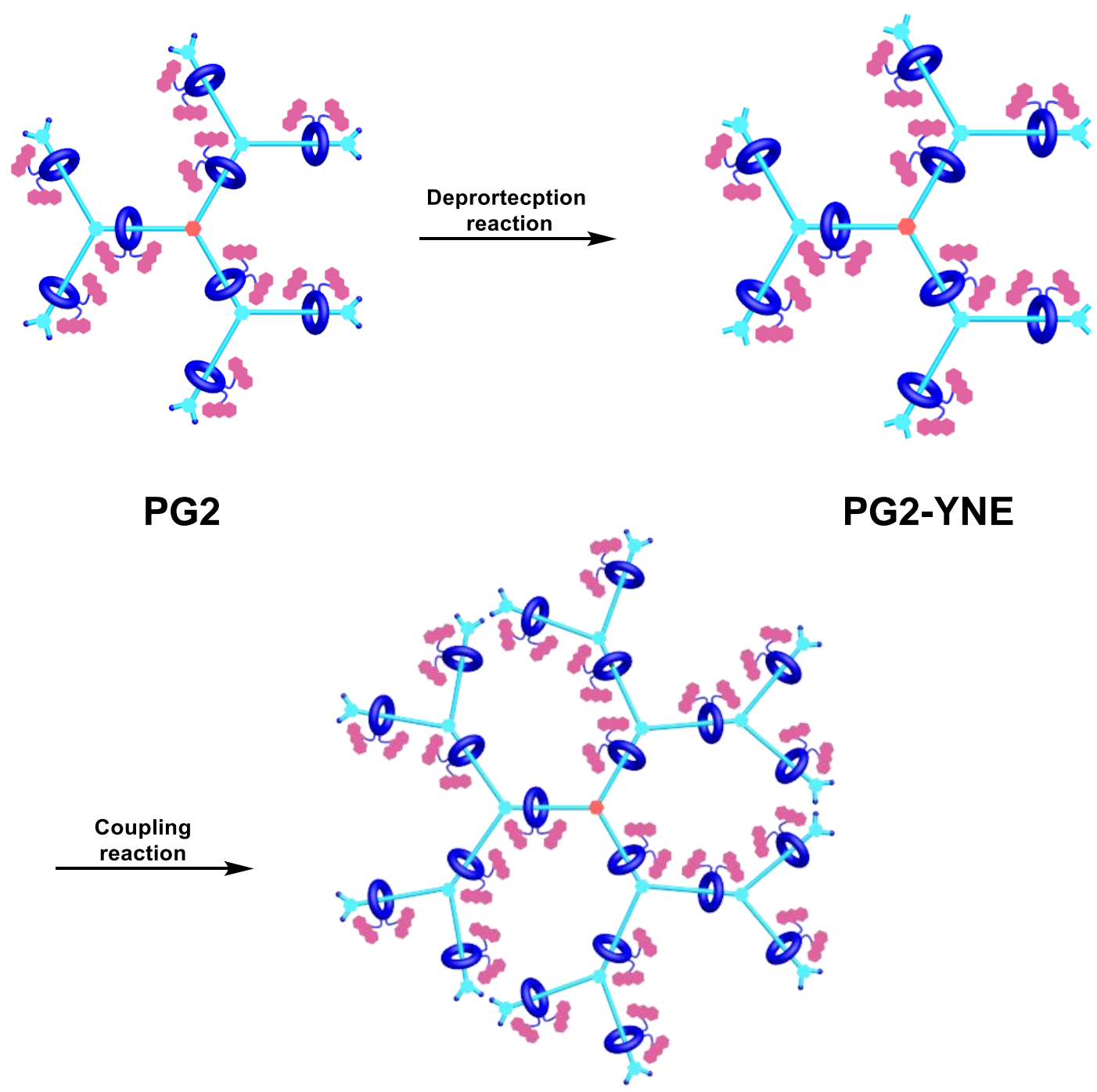

PG2-YNE

\section{PG3}

Synthesis of the rotaxane-branched dendrimer PG3: A reaction flask was charged with a THF solution of PG2 (100 mg, $0.0048 \mathrm{mmol})$ and then a THF solution of $\mathrm{Bu}_{4} \mathrm{NF} \cdot 3 \mathrm{H}_{2} \mathrm{O}(36 \mathrm{mg}, 0.11 \mathrm{mmol})$ was added dropwise into the reaction flask, the reaction mixture was stirred at room temperature for $2 \mathrm{~h}$. The obtained residue was washed by water, then dried with $\mathrm{Na}_{2} \mathrm{SO}_{4}$ and concentrated. The residue was further purified by column chromatography $\left(\mathrm{SiO}_{2} ; \mathrm{DCM}\right)$ and preparative gel permeation chromatography (GPC) to afford a pale-yellow solid PG2-YNE. Then the obtained PG2-YNE (58mg, $0.0031 \mathrm{mmol}$ ) and [2]rotaxane R-AN (102 mg, $0.048 \mathrm{mmol}$ ) were added in a Schlenk flask, the Schlenk flask was then evacuated and back-filled with $\mathrm{N}_{2}$ three times. Next, the mixture solvent of degassed $\mathrm{Et}_{2} \mathrm{NH}$ and $\mathrm{CH}_{2} \mathrm{Cl}_{2}(\mathrm{v} / \mathrm{v}, 10 / 10 \mathrm{~mL}$ ) was added via syringe. Subsequently, a catalytic amount of $\mathrm{CuI}$ was added under an 
inert atmosphere. The reaction was stirred overnight at room temperature. The solvent was evaporated and the residue was purified by column chromatography $\left(\mathrm{SiO}_{2}\right.$; DCM/EA) and preparative gel permeation chromatography (GPC) to yield a pale yellow solid PG3 (118 mg, 81\%). ${ }^{1} \mathrm{H}$ NMR (500 MHz, $\left.\mathrm{CD}_{2} \mathrm{Cl}_{2}\right): \delta 8.23-8.35(\mathrm{~m}), 8.00-$ $8.05(\mathrm{~m}), 7.65(\mathrm{~m}), 7.46-7.50(\mathrm{~m}), 7.18-7.23(\mathrm{~m}), 6.89-7.09(\mathrm{~m}), 6.73-6.74(\mathrm{~m}), 4.33-$ $4.36(\mathrm{~m}), 4.18-4.25(\mathrm{~m}), 4.01-4.02(\mathrm{~m}), 3.76-3.94(\mathrm{~m}), 2.99(\mathrm{~m}), 2.57(\mathrm{~m}), 2.13-2.43$ (m), $1.68(\mathrm{~m}), 1.53(\mathrm{~m}), 1.11-1.29(\mathrm{~m}),, 0.81(\mathrm{~m}), 0.69(\mathrm{~m}),-0.04(\mathrm{~m}),-1.31(\mathrm{~m}),-1.47$ (m) -1.73(m), -1.82 (m). ${ }^{31} \mathrm{P}$ NMR (202 MHz, $\left.\mathrm{CD}_{2} \mathrm{Cl}_{2}\right): \delta 11.64 .{ }^{13} \mathrm{C} \mathrm{NMR}(126 \mathrm{MHz}$, $\left.\mathrm{CD}_{2} \mathrm{Cl}_{2}\right): \delta 158.51,155.82,154.88,152.99,152.92,152.86,152.59,152.51,152.23$, $152.13,152.10,151.96,151.95,151.89,151.85,151.80,151.55,151.51,151.46$, $142.36,134.07,134.04,133.37,130.85,130.62,130.56,130.52,130.35,130.22$, $130.16,130.04,130.00,129.88,127.11,127.02,126.85,126.81,126.70,126.30,126.27$, $125.54,123.98,123.91,123.77,123.63,123.44,116.98,116.36,116.04,115.78,115.71$, $115.64,114.75,114.71,114.65,107.93,92.41,77.44,77.39,77.30,70.31,69.64,69.50$, $58.72,58.57,57.96,57.86,57.78,57.72,56.97,56.90,56.88,41.45,40.87,32.54,32.10$, $31.66,31.60,31.02,30.65,30.60,30.52,29.38,29.18,29.09,28.70,28.47,28.35,27.85$, $26.88,19.96,18.08,17.94,17.81,12.84,9.71$.

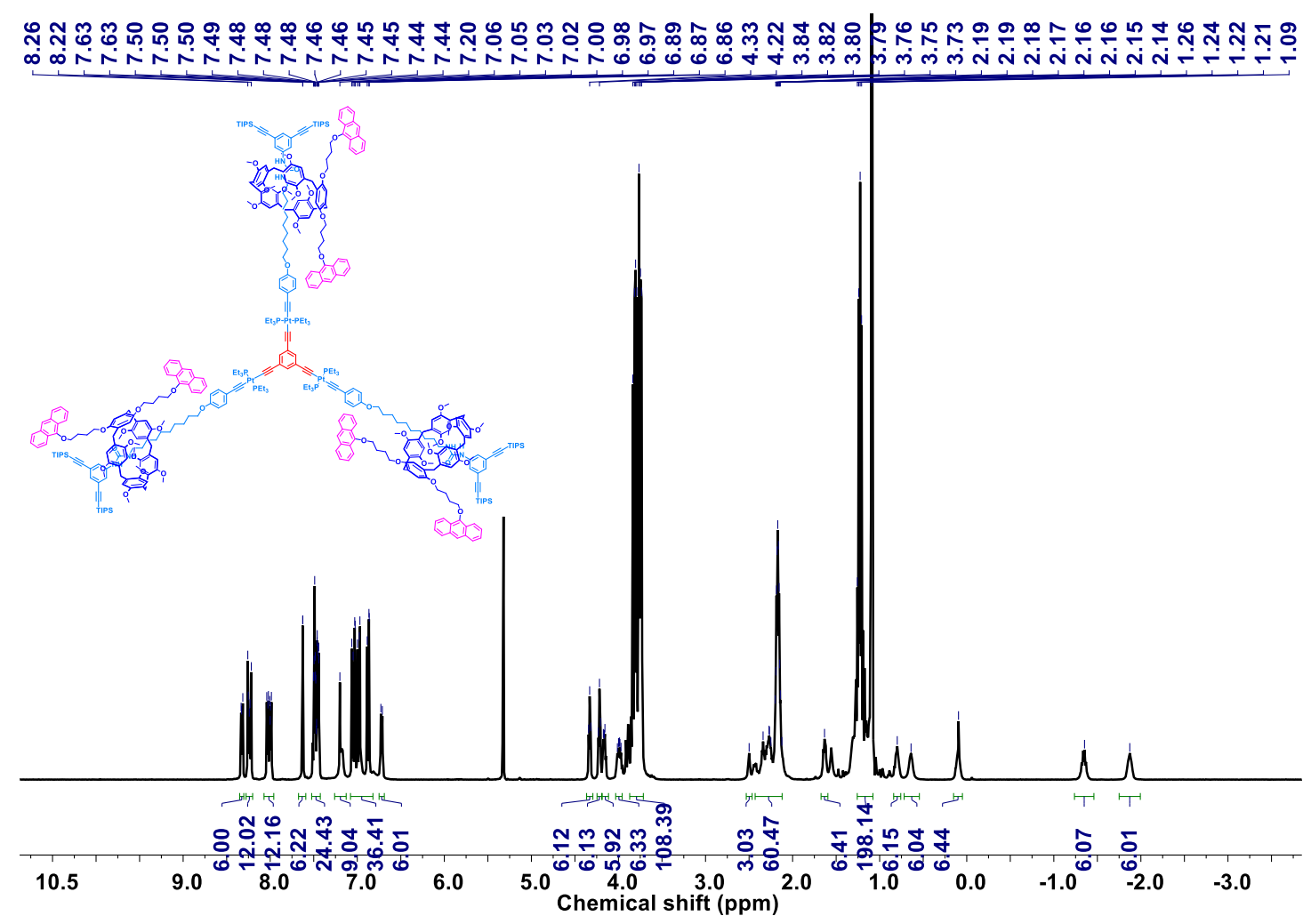

Figure S12. ${ }^{1} \mathrm{H}$ NMR spectrum $\left(\mathrm{CD}_{2} \mathrm{Cl}_{2}, 298 \mathrm{~K}, 500 \mathrm{MHz}\right)$ of PG1. 


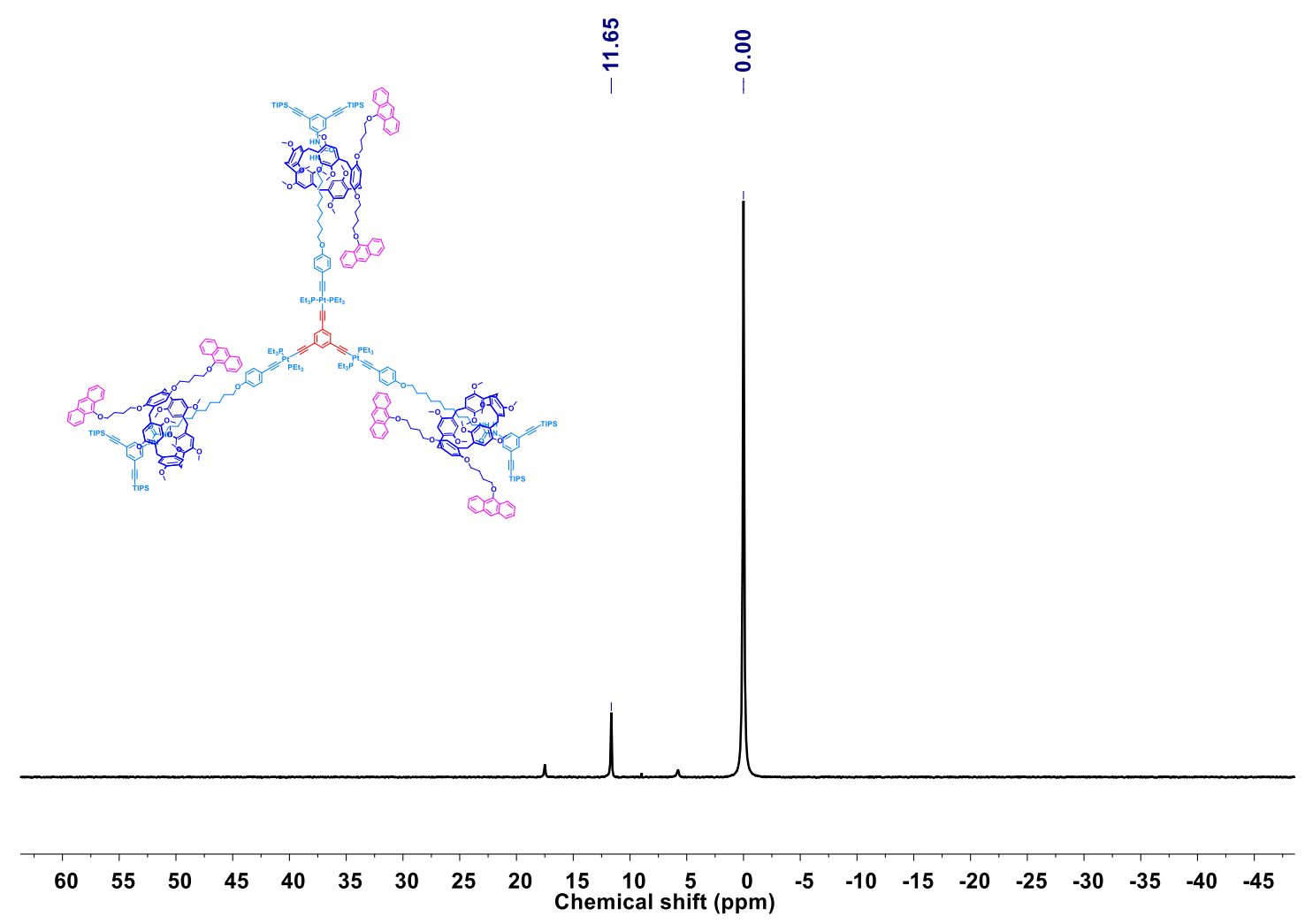

Figure S13. ${ }^{31} \mathrm{P}$ NMR spectrum $\left(\mathrm{CD}_{2} \mathrm{Cl}_{2}, 298 \mathrm{~K}, 202 \mathrm{MHz}\right)$ of PG1.

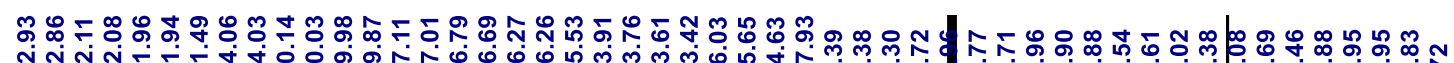

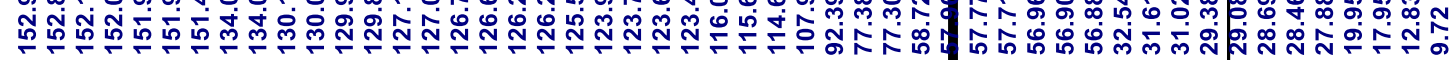

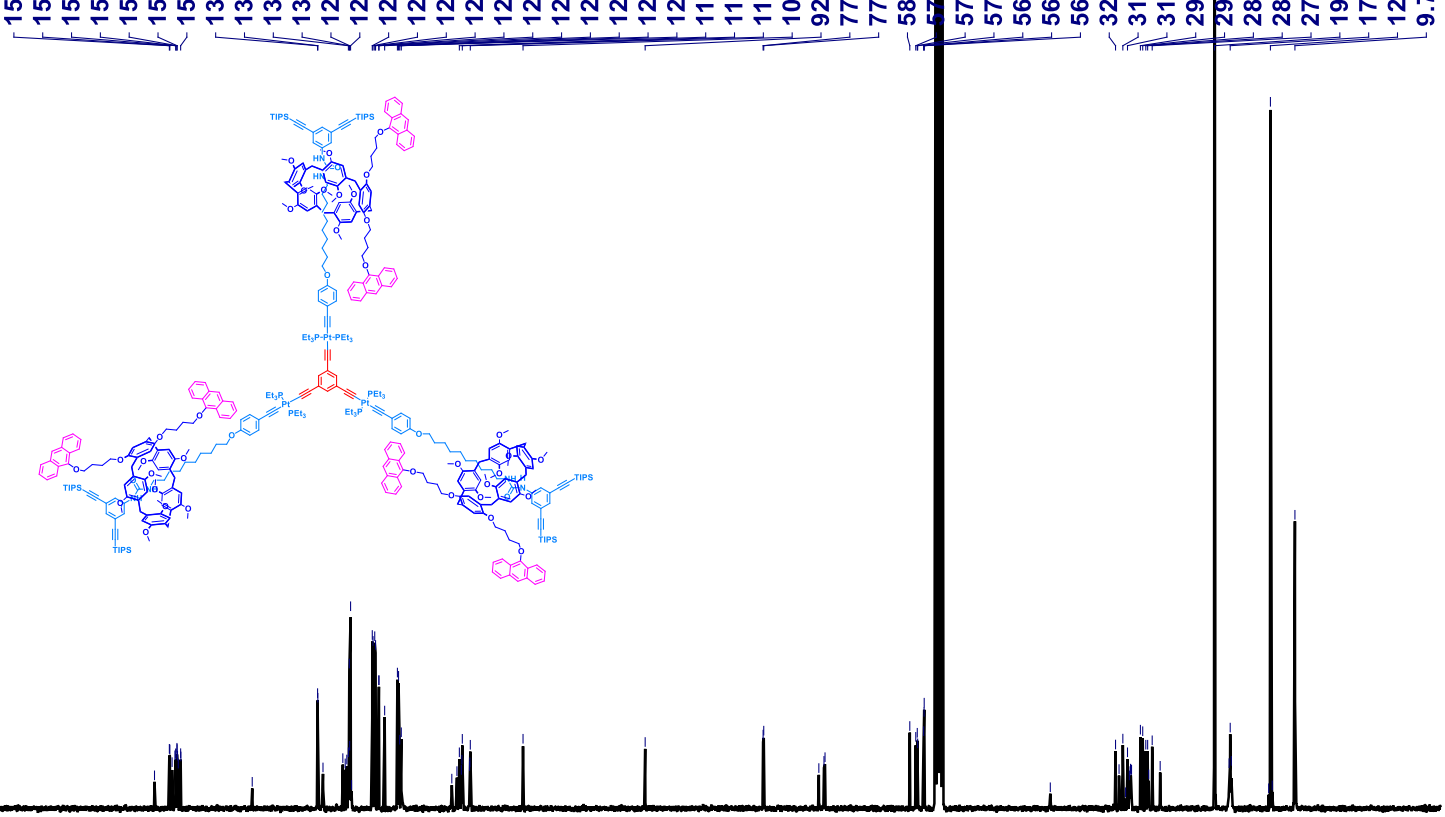

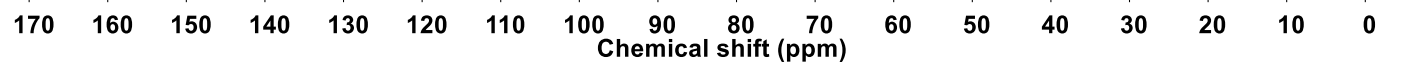

Figure S14. ${ }^{13} \mathrm{C}$ NMR spectrum $\left(\mathrm{CD}_{2} \mathrm{Cl}_{2}, 298 \mathrm{~K}, 126 \mathrm{MHz}\right)$ of PG1. 


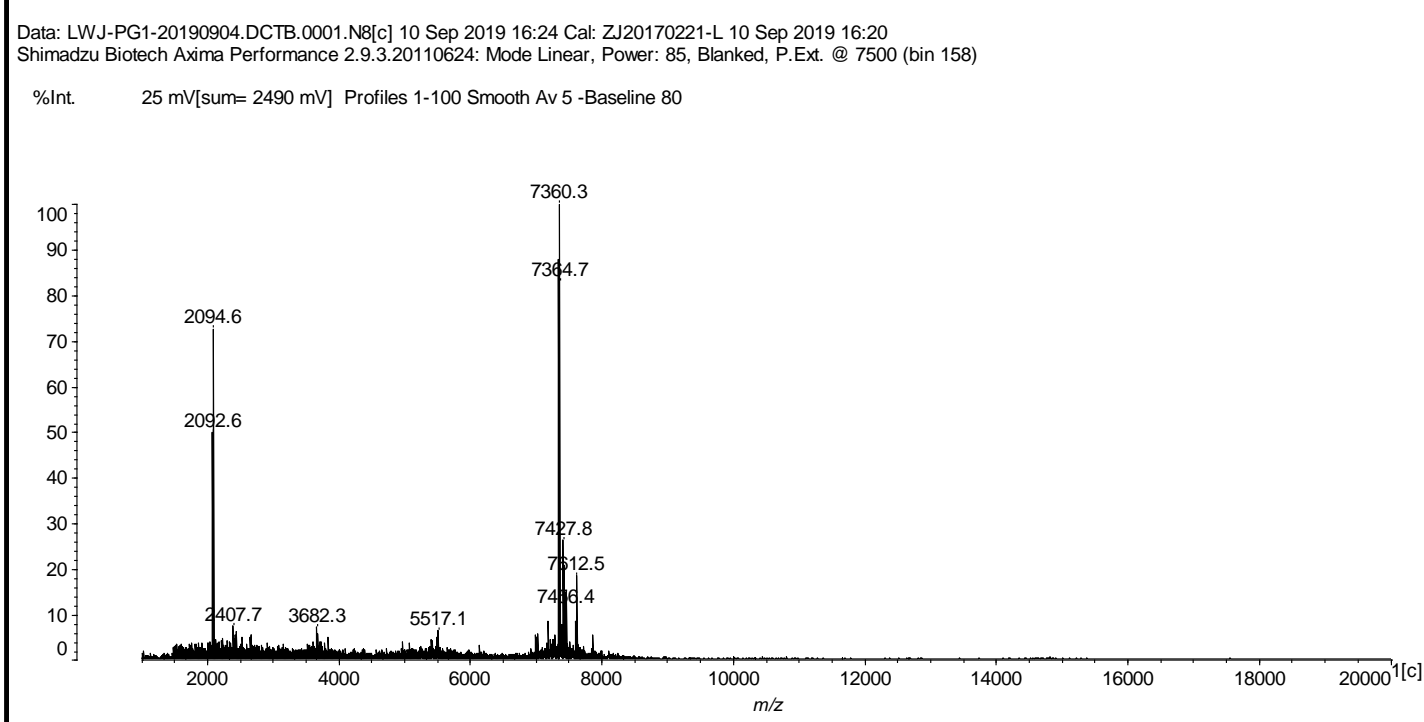

Figure S15. LRMS (MALDI-TOF-MS) spectrum of PG1.

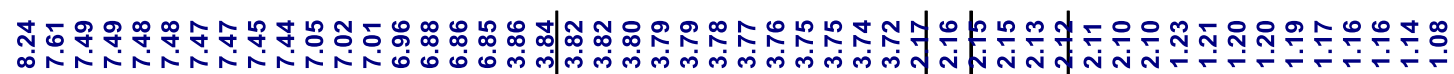

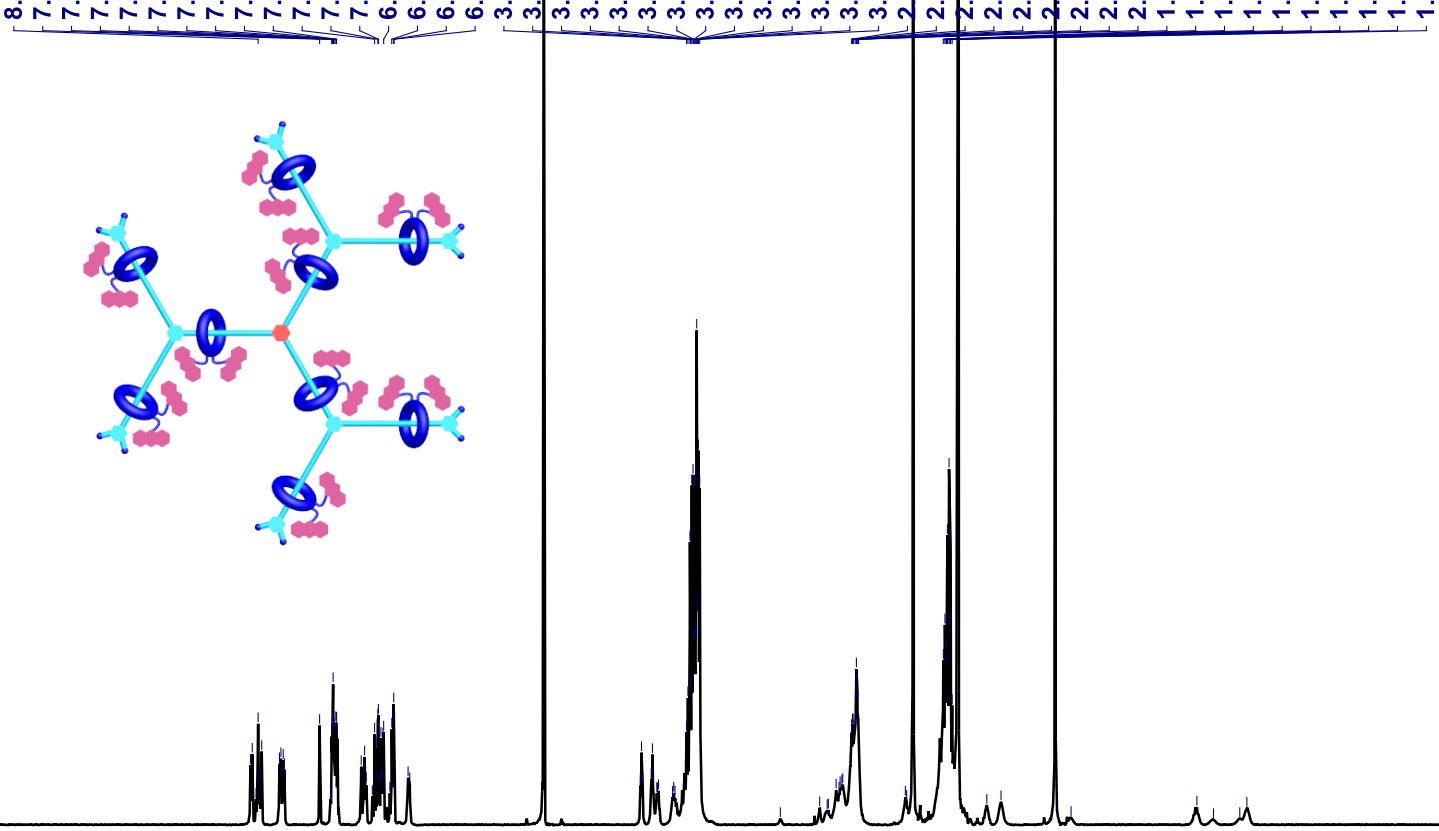

10.5

$\begin{array}{llll}9.0 & 8.0 & 7.0 & 6.0\end{array}$

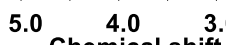

2.

Figure S16. ${ }^{1} \mathrm{H}$ NMR spectrum $\left(\mathrm{CD}_{2} \mathrm{Cl}_{2}, 298 \mathrm{~K}, 500 \mathrm{MHz}\right)$ of PG2. 


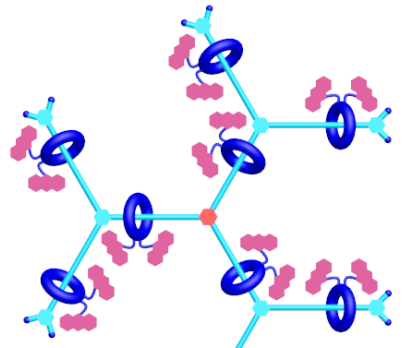

o)

$\begin{array}{lllllllllllll}60 & 55 & 50 & 45 & 40 & 35 & 30 & 25 & 20 & 15 & 10 & 5 & 0\end{array}$

Figure S17. ${ }^{31} \mathrm{P}$ NMR spectrum $\left(\mathrm{CD}_{2} \mathrm{Cl}_{2}, 298 \mathrm{~K}, 202 \mathrm{MHz}\right)$ of PG2.

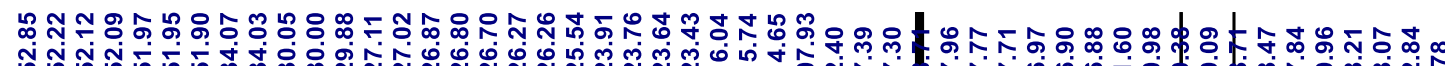

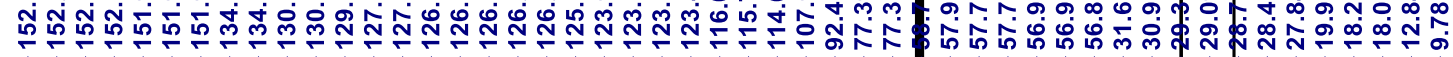

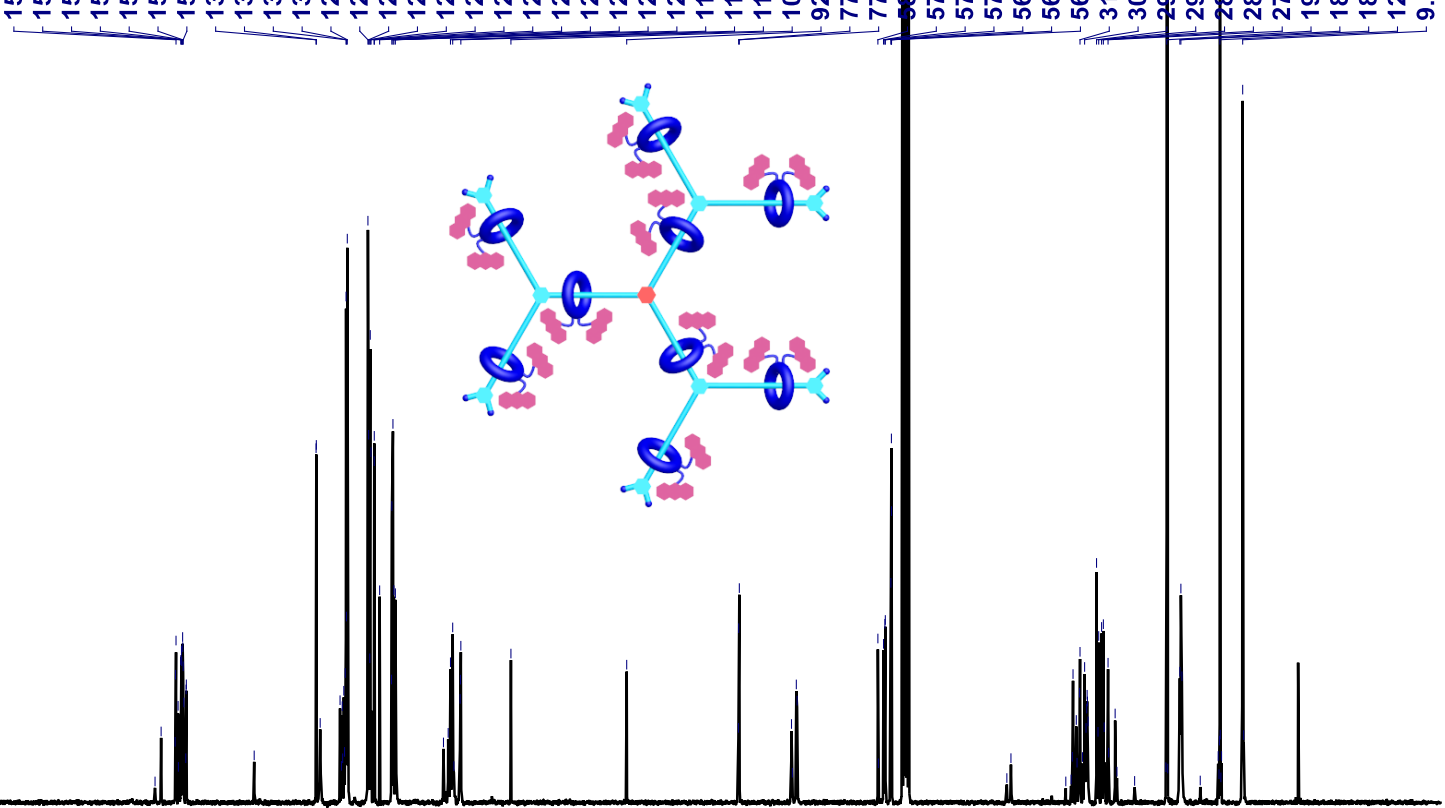

$\begin{array}{lllllllllllllllllll}170 & 160 & 150 & 140 & 130 & 120 & 110 & 100 & \begin{array}{c}90 \\ \text { Chemical shift }\end{array} \underset{(\mathrm{ppm})}{70}\end{array}$

Figure S18. ${ }^{13} \mathrm{C}$ NMR spectrum $\left(\mathrm{CD}_{2} \mathrm{Cl}_{2}, 298 \mathrm{~K}, 126 \mathrm{MHz}\right)$ of PG2. 


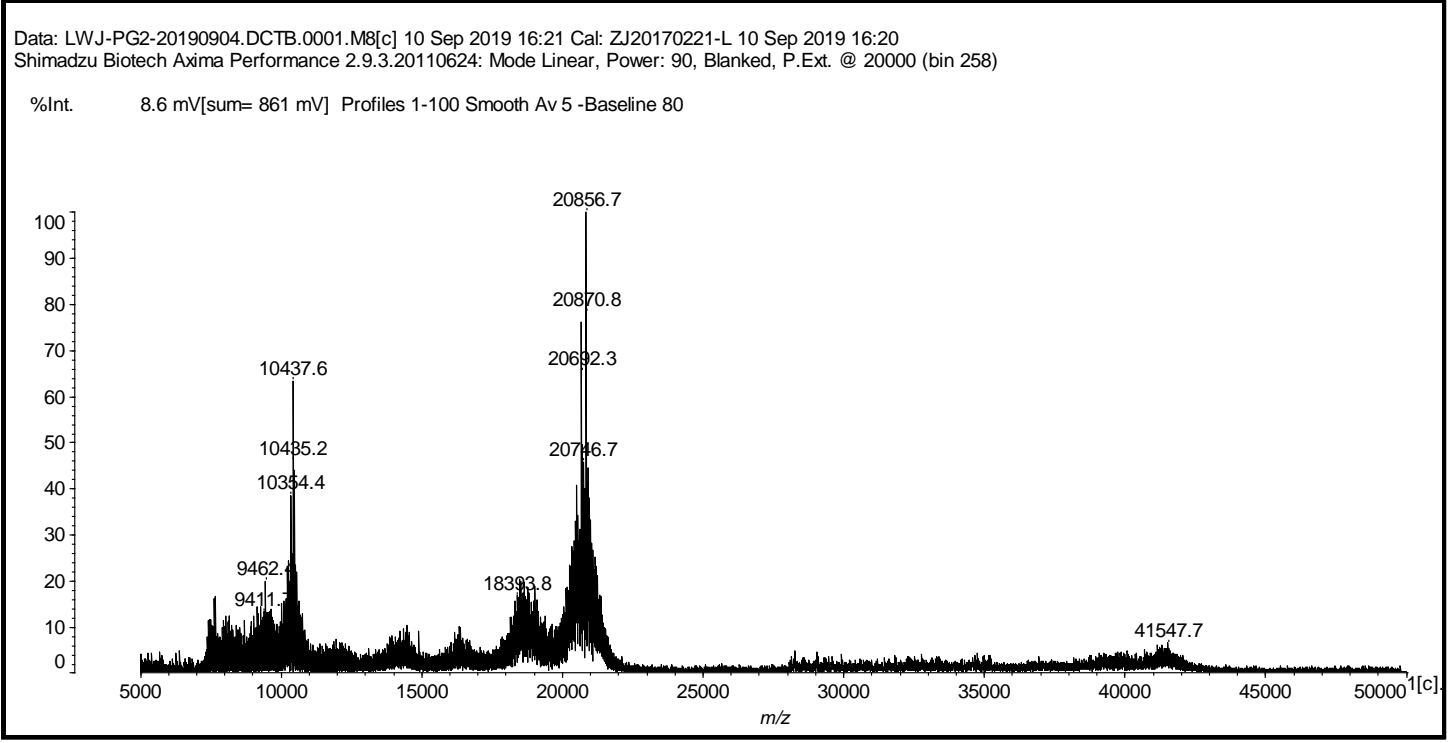

Figure S19. LRMS (MALDI-TOF-MS) spectrum of PG2.

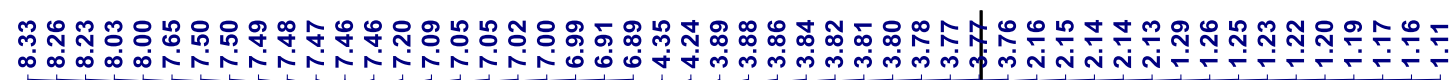
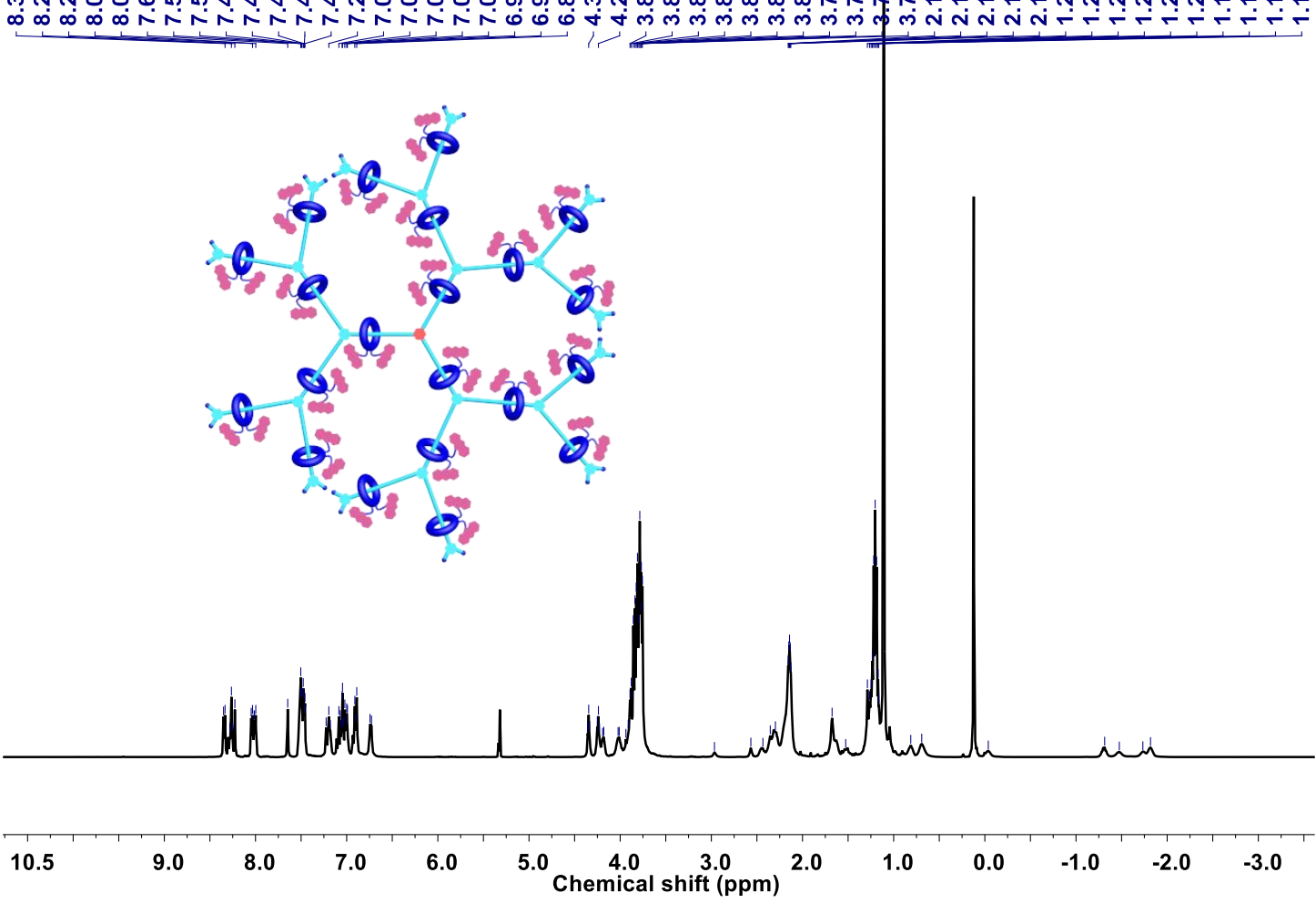

Figure S20. ${ }^{1} \mathrm{H}$ NMR spectrum $\left(\mathrm{CD}_{2} \mathrm{Cl}_{2}, 298 \mathrm{~K}, 500 \mathrm{MHz}\right)$ of $\mathbf{P G 3}$. 


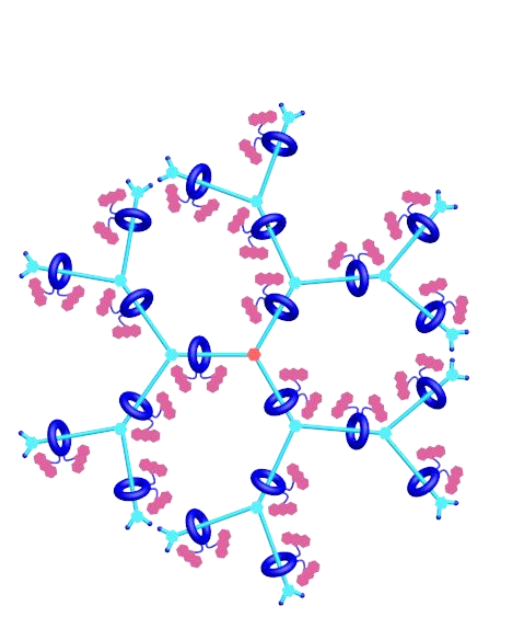

$\stackrel{+}{\stackrel{0}{i}} \quad \stackrel{\circ}{i}$

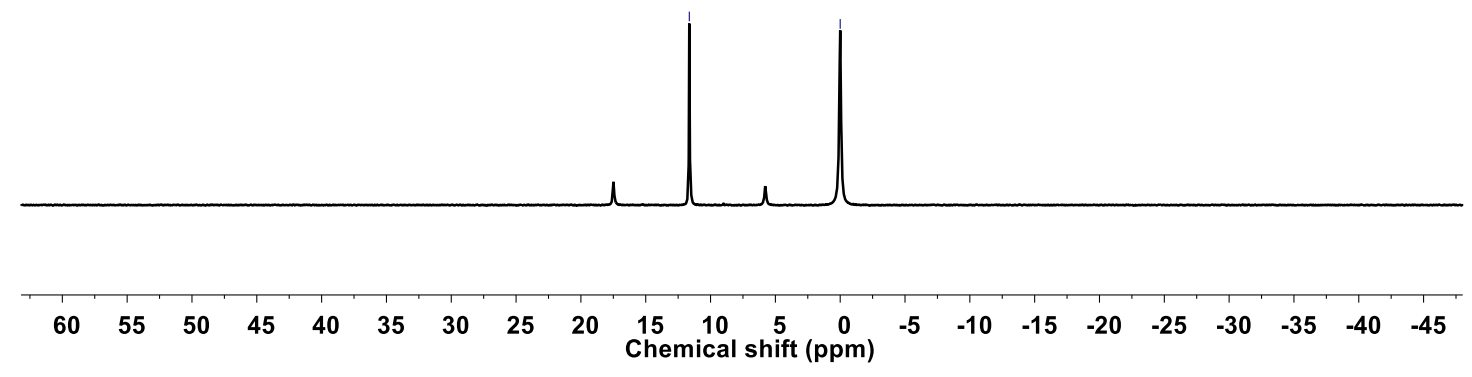

Figure S21. ${ }^{31} \mathrm{P}$ NMR spectrum $\left(\mathrm{CD}_{2} \mathrm{Cl}_{2}, 298 \mathrm{~K}, 202 \mathrm{MHz}\right)$ of PG3.

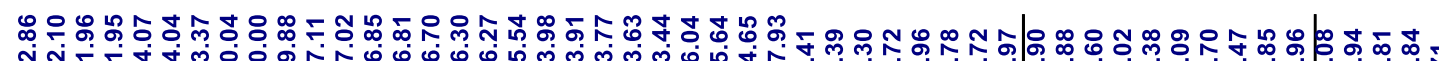

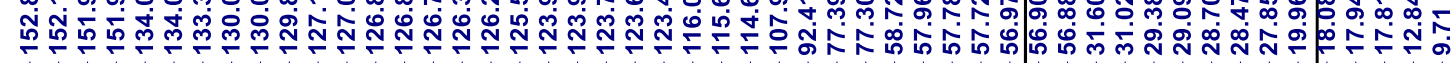

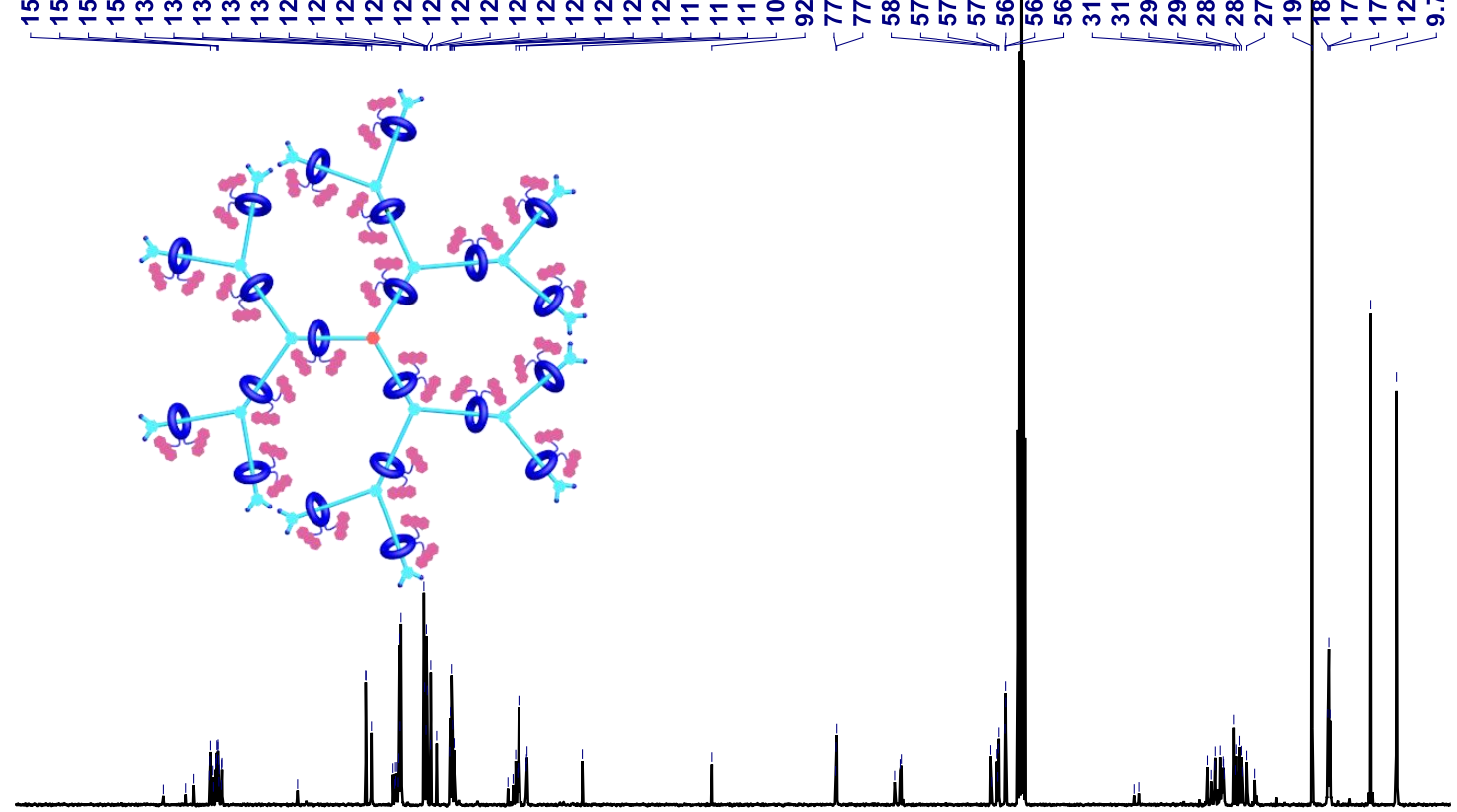

$\begin{array}{llllllllllllllll}170 & 160 & 150 & 140 & 130 & 120 & 110 & \begin{array}{c}100 \\ \text { Chemical shift }(\mathrm{ppm})\end{array} & 90 & 60 & 50 & 40 & 30 & 20 & 10\end{array}$

Figure S22. ${ }^{13} \mathrm{C}$ NMR spectrum $\left(\mathrm{CD}_{2} \mathrm{Cl}_{2}, 298 \mathrm{~K}, 126 \mathrm{MHz}\right)$ of PG3. 


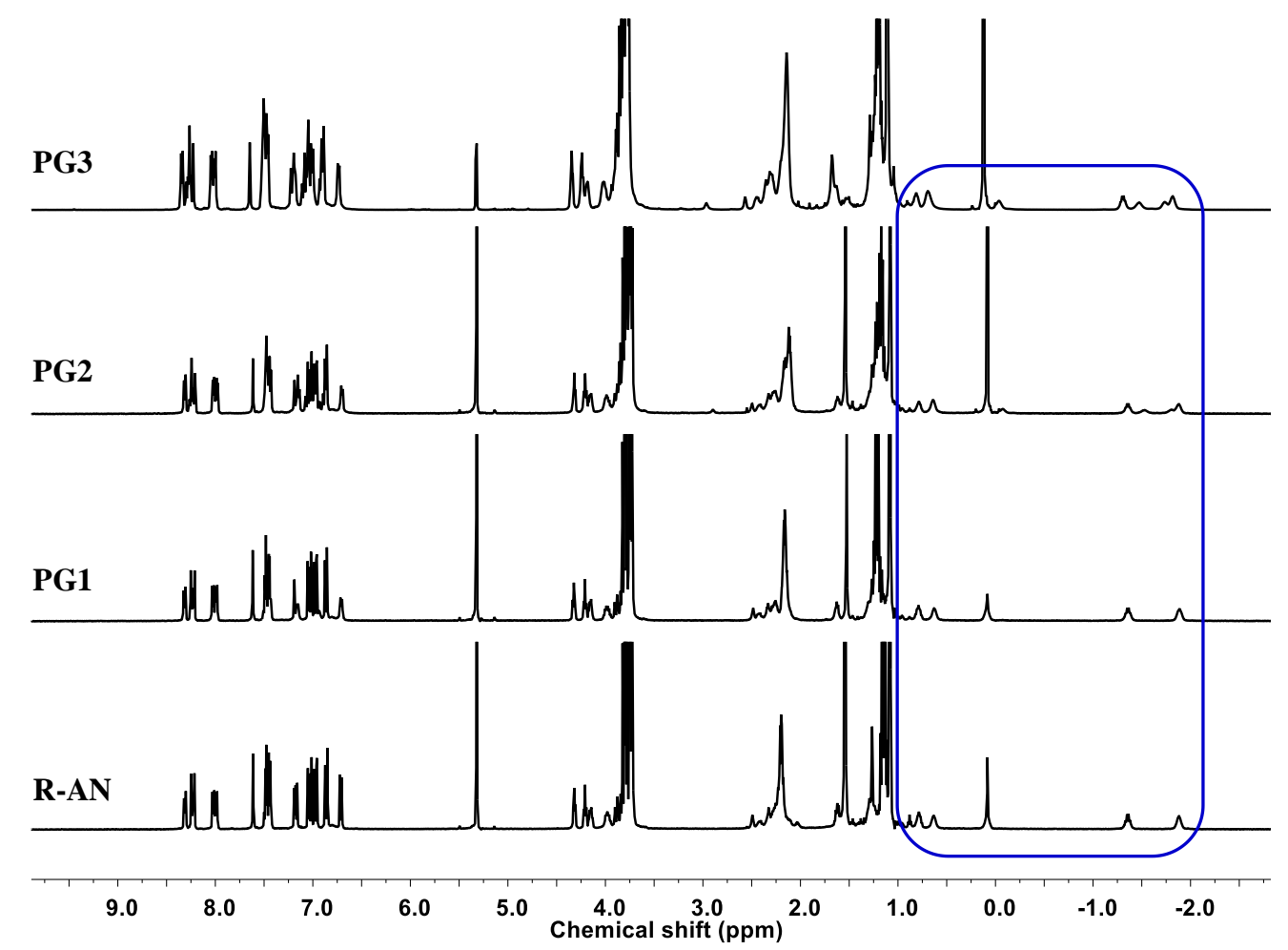

Figure S23. ${ }^{1} \mathrm{H}$ NMR spectra $\left(\mathrm{CD}_{2} \mathrm{Cl}_{2}, 298 \mathrm{~K}, 500 \mathrm{MHz}\right)$ of the rotaxane-branched dendrimers PG1-PG3 and [2] rotaxane R-AN.

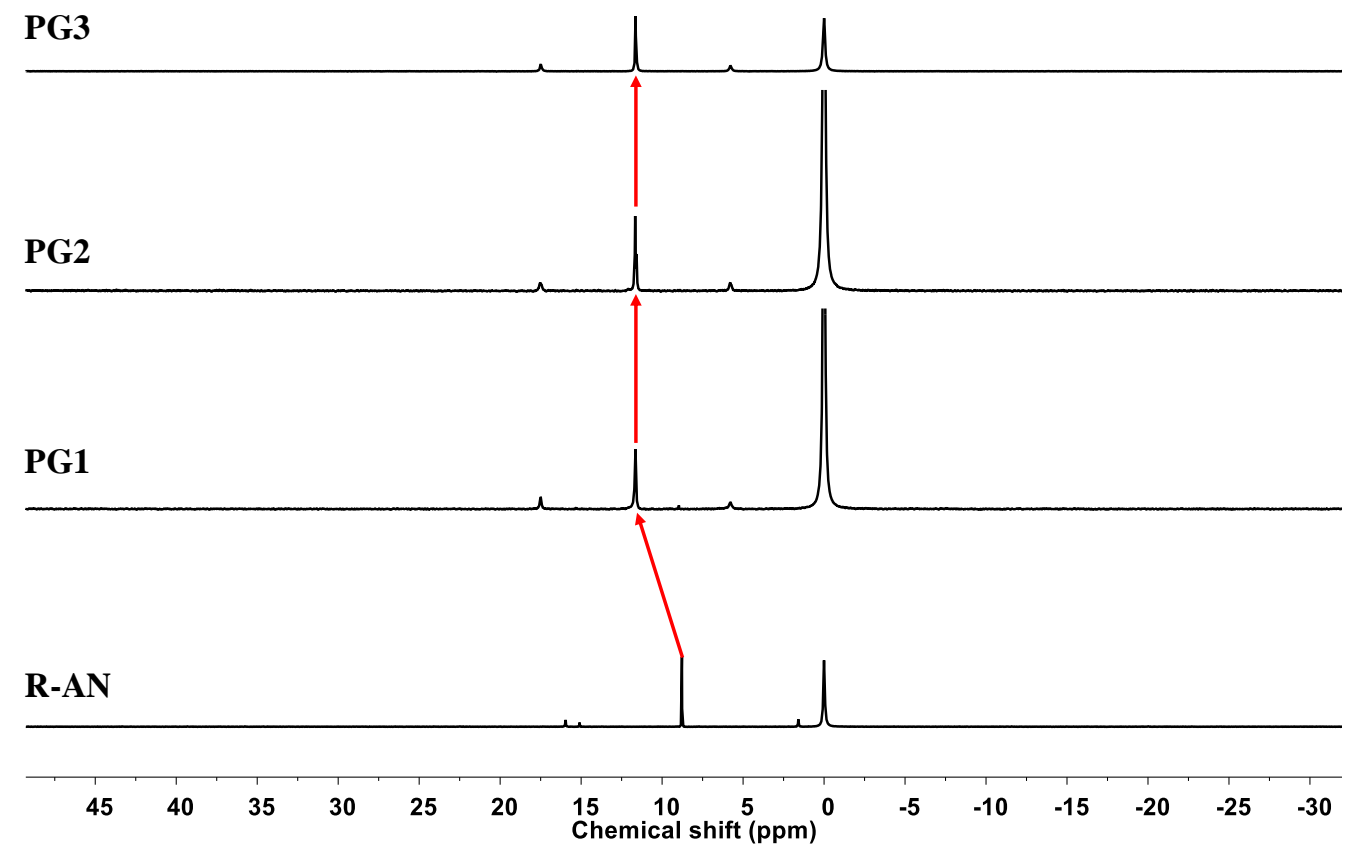

Figure S24. ${ }^{31} \mathrm{P}$ NMR spectra $\left(\mathrm{CD}_{2} \mathrm{Cl}_{2}, 298 \mathrm{~K}, 202 \mathrm{MHz}\right)$ of the rotaxane-branched dendrimers PG1-PG3 and [2] rotaxane R-AN. 


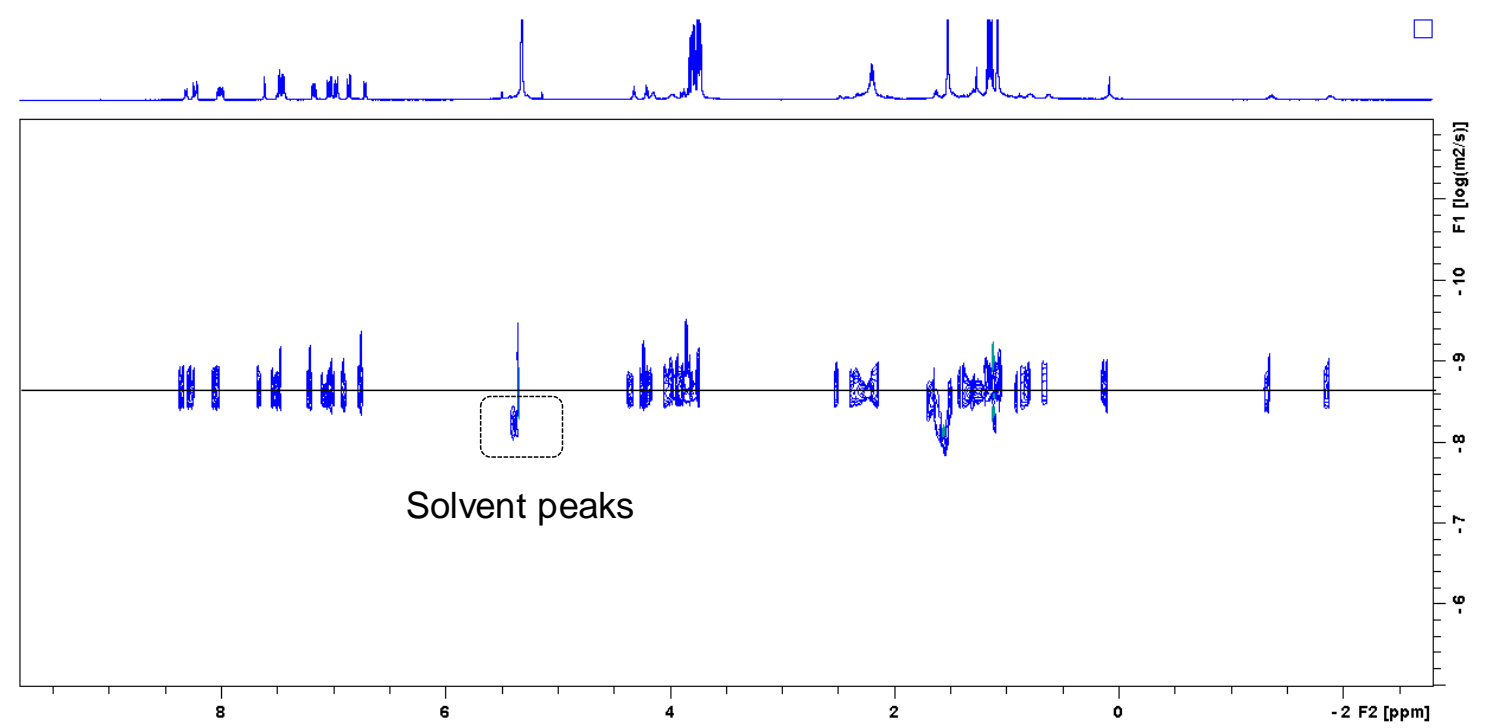

Figure S25. 2-D DOSY spectrum $\left(\mathrm{CD}_{2} \mathrm{Cl}_{2}, 298 \mathrm{~K}, 500 \mathrm{MHz}\right)$ of [2] rotaxane R-AN, the diffusion coefficient $D=(2.29 \pm 0.10) \times 10^{-9} \mathrm{~m}^{2} \mathrm{~s}^{-1}$.

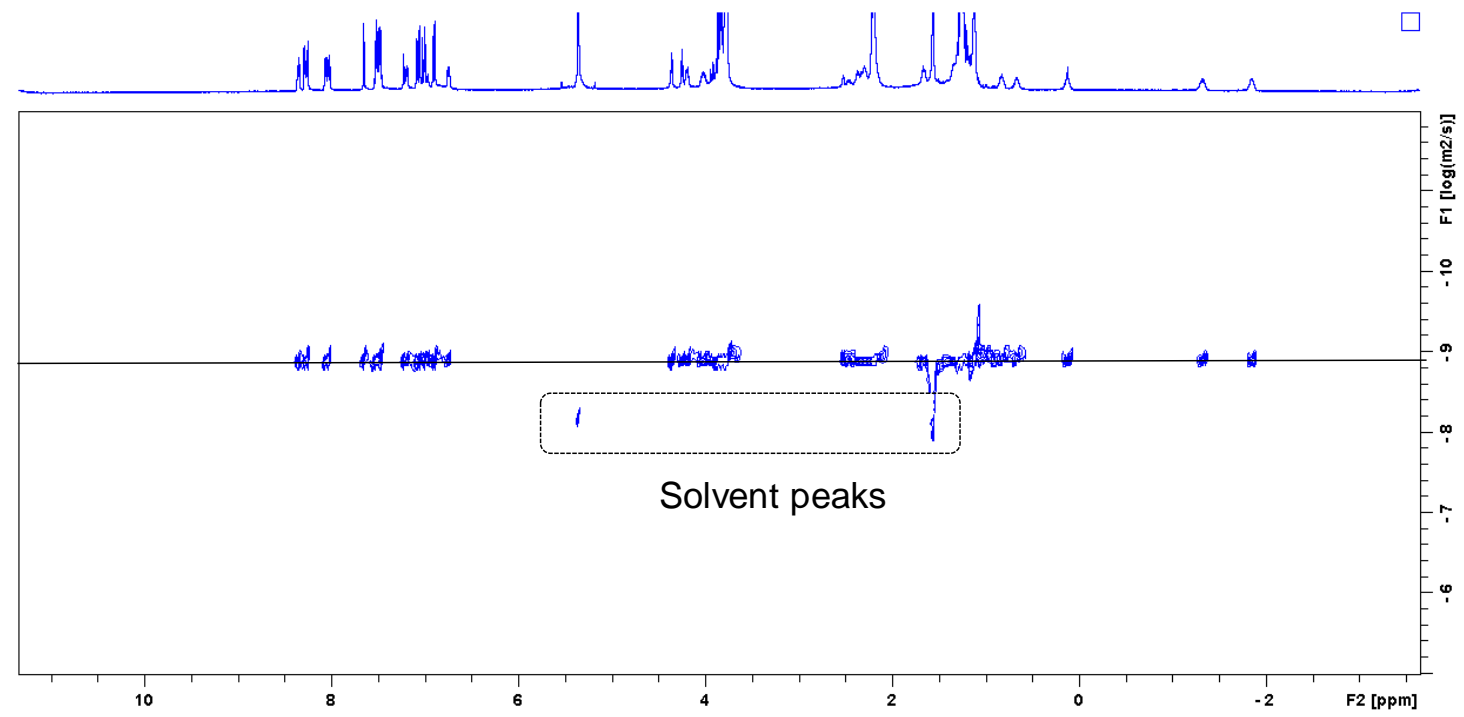

Figure S26. 2-D DOSY spectrum $\left(\mathrm{CD}_{2} \mathrm{Cl}_{2}, 298 \mathrm{~K}, 500 \mathrm{MHz}\right)$ of PG1, the diffusion coefficient $D=(1.38 \pm 0.12) \times 10^{-9} \mathrm{~m}^{2} \mathrm{~s}^{-1}$. 


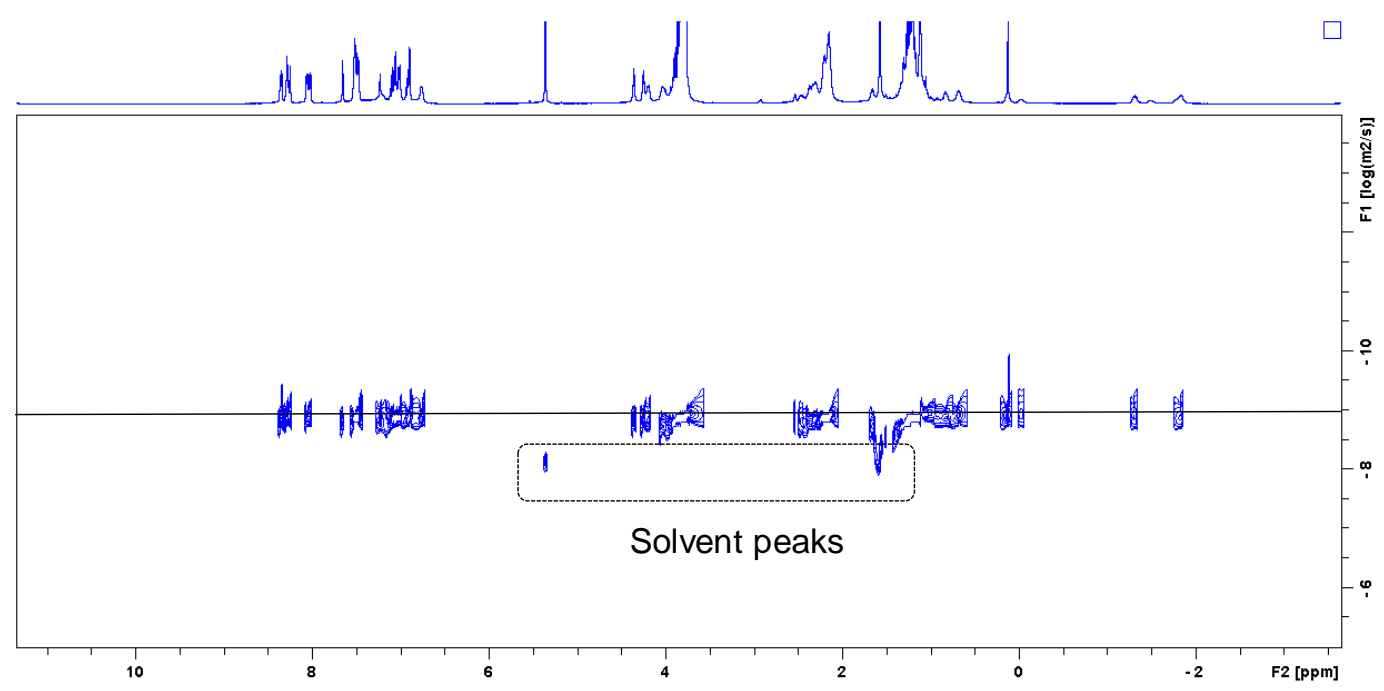

Figure S27. 2-D DOSY spectrum $\left(\mathrm{CD}_{2} \mathrm{Cl}_{2}, 298 \mathrm{~K}, 500 \mathrm{MHz}\right)$ of $\mathbf{P G 2}$, the diffusion coefficient $D=(1.02 \pm 0.10) \times 10^{-9} \mathrm{~m}^{2} \mathrm{~s}^{-1}$.

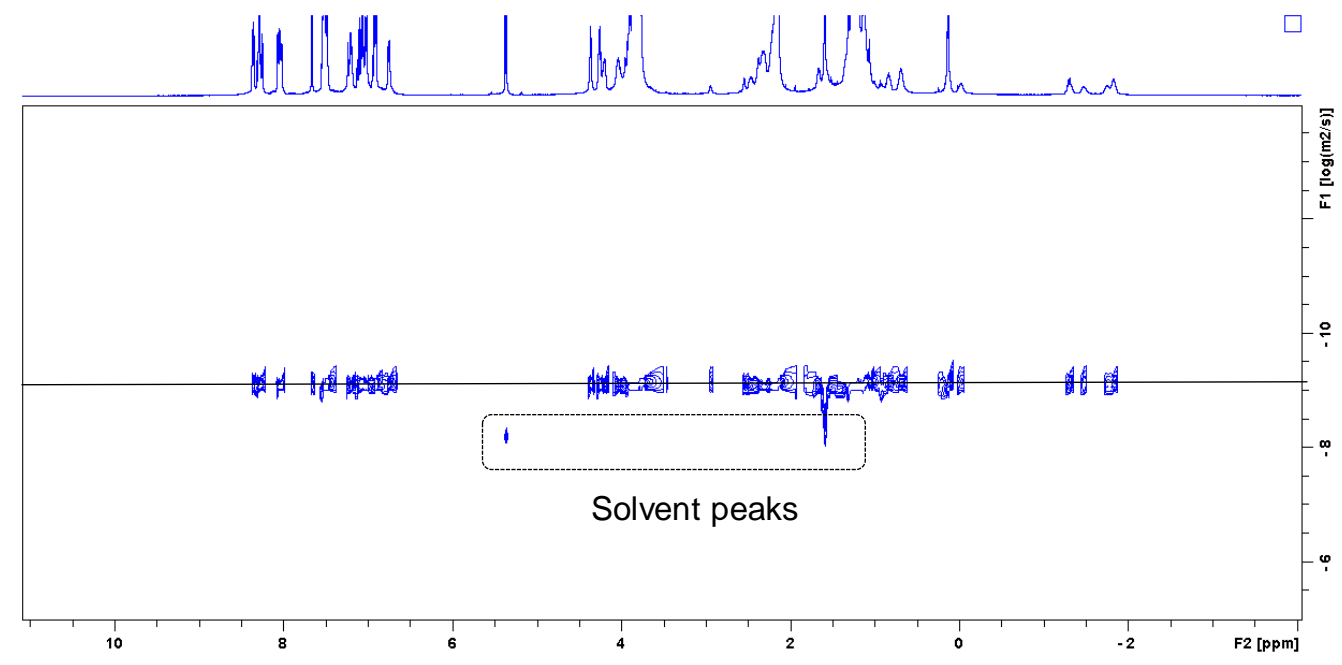

Figure S28. 2-D DOSY spectrum $\left(\mathrm{CD}_{2} \mathrm{Cl}_{2}, 298 \mathrm{~K}, 500 \mathrm{MHz}\right)$ of PG3, the diffusion coefficient $D=(6.76 \pm 0.15) \times 10^{-10} \mathrm{~m}^{2} \mathrm{~s}^{-1}$.

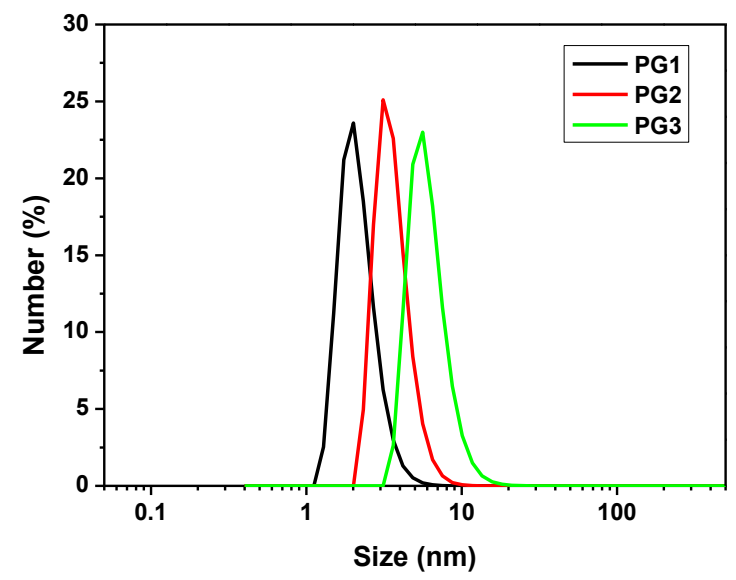

Figure S29. DLS spectra of rotaxane-branched dendrimer PGn $(n=1,2,3)\left(10^{-5} \mathrm{M}\right)$. The average hydrodynamic size was $2.2 \pm 0.1 \mathrm{~nm}, 3.6 \pm 0.2 \mathrm{~nm}$ and $6.2 \pm 0.2 \mathrm{~nm}$ for PG1, PG2 and PG3 respectively. 


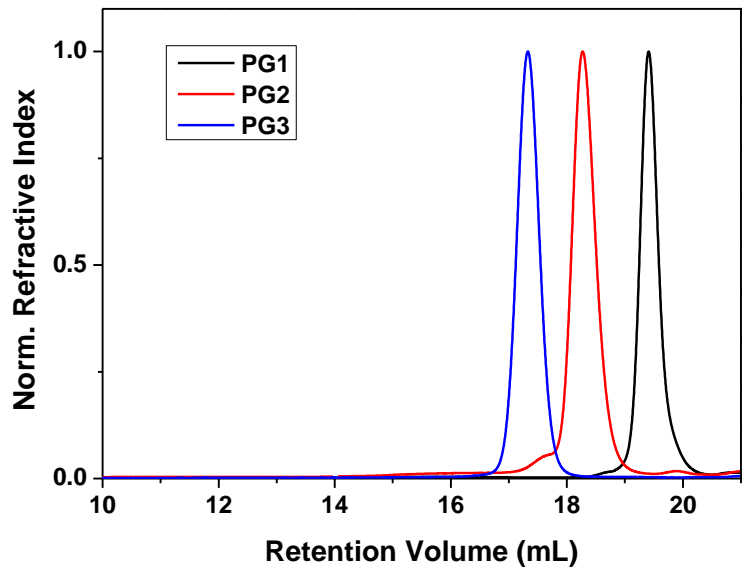

Figure S30. GPC spectra of rotaxane-branched dendrimers PG1-PG3. For PG1, PDI $=1.02, M_{\mathrm{n}}=7,490$. For PG2, PDI $=1.02, M_{\mathrm{n}}=17,599$. For PG3, PDI $=1.04, M_{\mathrm{n}}=$ 32,945 .

(a)

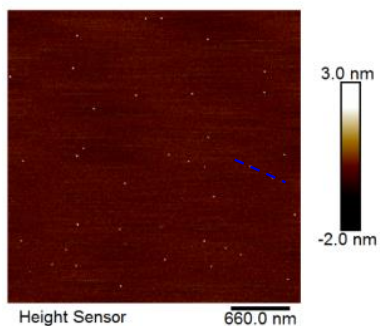

(d)

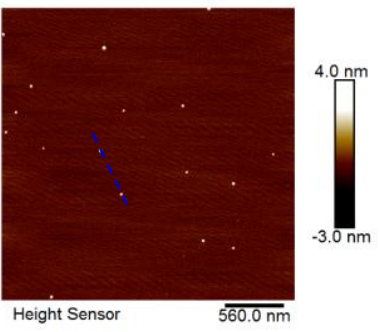

(g)

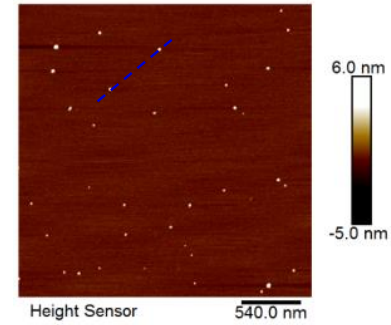

(b)

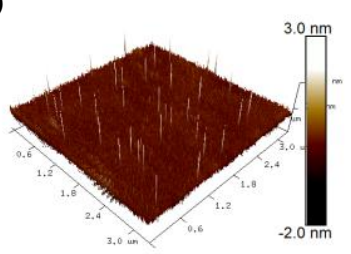

(e)

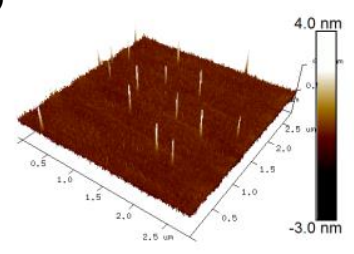

(h)

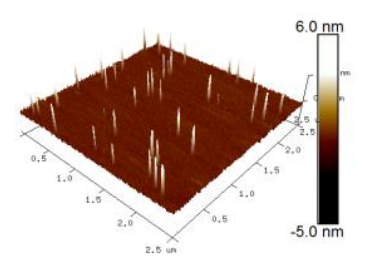

(c)

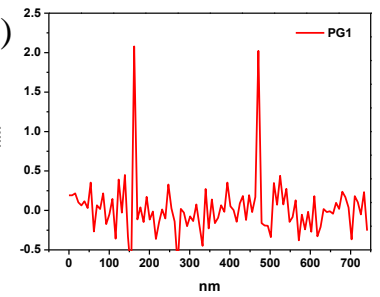

(f)

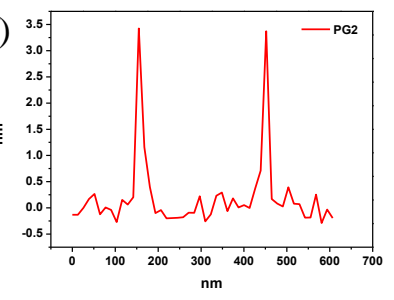

(i)

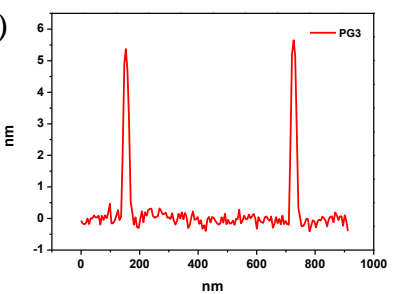

Figure S31. AFM images of the rotaxane-branched dendrimers. (a-b) PG1; (c) the height range of PG1 is $2.1 \pm 0.1 \mathrm{~nm}$; (d-e) PG2; (f) the height range of PG2 is $3.4 \pm$ $0.1 \mathrm{~nm}$; (g-h) PG3; (i) the height range of PG3 is $5.5 \pm 0.2 \mathrm{~nm}$. 


\section{Section D. Controllable generation of singlet oxygen}

An ultraviolet lamp (ZF-7A, $365 \mathrm{~nm}, 16 \mathrm{~W}, 220 \mathrm{~V} / 50 \mathrm{~Hz}$ ) was used as the light source of singlet oxygen generation. Singlet oxygen sensor green (SOSG) reagent (Life Technologies) was employed for the detection of singlet oxygen. All samples were prepared in THF solutions with same concentration of anthracene unit $(0.042 \mathrm{mM})$. To $2 \mathrm{~mL}$ each of these solutions, SOSG stock solution $(20 \mu \mathrm{L}$ at $1 \mathrm{mM})$ was added (final concentration $=10 \mu \mathrm{M}$ ) before fluorescence measurement.

For a typical measurement, fluorescence intensity was acquired on a Shimadzu RF6000 fluorescence spectrophotometer with excitation at $504 \mathrm{~nm}$ and emission at 550 $\mathrm{nm}$ (slit width $15 \mathrm{~nm} / 15 \mathrm{~nm}$ for ex/em) in air. Fluorescence was measured after irradiation by lamp for 0 (as background), $2 \mathrm{~min}, 4 \mathrm{~min}, 6 \mathrm{~min}, 8 \mathrm{~min}, 10 \mathrm{~min}, 15 \mathrm{~min}$, $20 \mathrm{~min}$ and $25 \mathrm{~min}, 30 \mathrm{~min}$.

As the light intensity and photosensitizer concentration are fixed, for the photoreaction, we can assume that $\left[\mathrm{PS}^{*}\right]$ (the concentration of the excited state of the photosensitizer) is a constant. Therefore, we have singlet oxygen generation rate equation:

$$
\frac{d\left[{ }^{1} O_{2}\right]}{d t}=-\frac{d\left[O_{2}\right]}{d t}=k\left[\mathrm{PS}^{*}\right]\left[O_{2}\right]=k^{*}\left[O_{2}\right]
$$

Where $k^{*}=k\left[\mathrm{PS}^{*}\right]$. Here we have a coupled reaction of SOSG to consume singlet oxygen:

$$
\frac{d\left[S O S G^{*}\right]}{d t}=k_{2}\left[{ }^{1} O_{2}\right][S O S G]
$$

Where $\left[S O S G^{*}\right]$ is the concentration of reacted form of SOSG.

Applying steady state assumption on $\left[{ }^{1} \mathrm{O}_{2}\right]$, we have

$$
\begin{gathered}
\frac{d\left[{ }^{1} O_{2}\right]}{d t}=k^{*}\left[O_{2}\right]-k_{d}\left[{ }^{1} O_{2}\right]-k_{2}\left[{ }^{1} O_{2}\right][S O S G]=0 \\
{\left[{ }^{1} O_{2}\right]=-\frac{k^{*}\left[O_{2}\right]}{k_{d}+k_{2}[S O S G]}}
\end{gathered}
$$

Where $\mathrm{k}_{\mathrm{d}}$ is the rate constant of singlet oxygen loss (due to relaxation or quenched by other species in the solution). When $\mathrm{k}_{2}[S O S G]<<\mathrm{k}_{\mathrm{d}}$ and $\left[\mathrm{O}_{2}\right]$ is high,

$$
\begin{gathered}
{\left[{ }^{1} O_{2}\right] \approx-\frac{k^{*}\left[O_{2}\right]}{k_{d}}=\text { constant }} \\
\frac{d I_{F}}{d t} \propto \frac{d\left[S O S G^{*}\right]}{d t}=k_{1}[S O S G]
\end{gathered}
$$

Where $\mathrm{k}_{1}=\mathrm{k}_{2} \mathrm{k}^{*}[\mathrm{O} 2] / \mathrm{k}_{\mathrm{d}}$, and the fluorescence intensity is proportional to [SOSG*]:

$$
I_{F}=I_{0} \varphi_{f} \varepsilon S\left[S O S G^{*}\right]
$$

Where $I_{0}$ is the incident light intensity, $\varphi_{f}$ is the fluorescence quantum yield of SOSG*, $\varepsilon_{S}$ is the extinction coefficient of SOSG*, and $b$ is the light path length. We can integrate the equation to obtain the correlation of fluorescence intensity $I_{F}$ and irradiation time $t$ : 


$$
\begin{gathered}
\ln \frac{[S O S G]}{c_{0}(S O S G)}=-k_{1} t \\
I_{F}=A\left[1-e^{-k t}\right]
\end{gathered}
$$

Where $A$ and $k$ are fitting parameters,

$$
\begin{gathered}
A=I_{0} \varphi_{f} \varepsilon_{S} b c_{0}(S O S G) \\
k=\varphi_{\Delta} N_{i r} \varepsilon_{P S} b c(P S) k_{2} / k_{\mathrm{d}}
\end{gathered}
$$

Where $c_{0}(S O S G)$ is the initial SOSG concentration; $\varphi_{\Delta}$ is the quantum yield of singlet oxygen generation, $N_{i r}$ is the irradiation light intensity by photons per second, $\varepsilon_{P S}$ is the extinction coefficient of photosensitizer, $c(P S)$ is the photosensitizer concentration. Linear approximations are applied in above equations. By non-linear regression, we obtained a series of fit curves with the aforementioned function.

The summary of all fitting results are included in Table S2.

Table S2. Fitting parameters of singlet oxygen generation rate.

\begin{tabular}{cccc}
\hline & $\mathrm{R}^{2}$ & $A k$ & Normalized yield \\
\hline AN & 0.9893 & $18.1 \pm 2.4$ & 0.5 \\
\hline R-AN & 0.9850 & $21.5 \pm 2.2$ & 0.6 \\
\hline PG1 & 0.9908 & $33.3 \pm 1.6$ & 1.0 \\
\hline PG2 & 0.9956 & $82.1 \pm 5.0$ & 2.5 \\
\hline PG3 & 0.9850 & $241.5 \pm 4.2$ & 7.3 \\
\hline
\end{tabular}
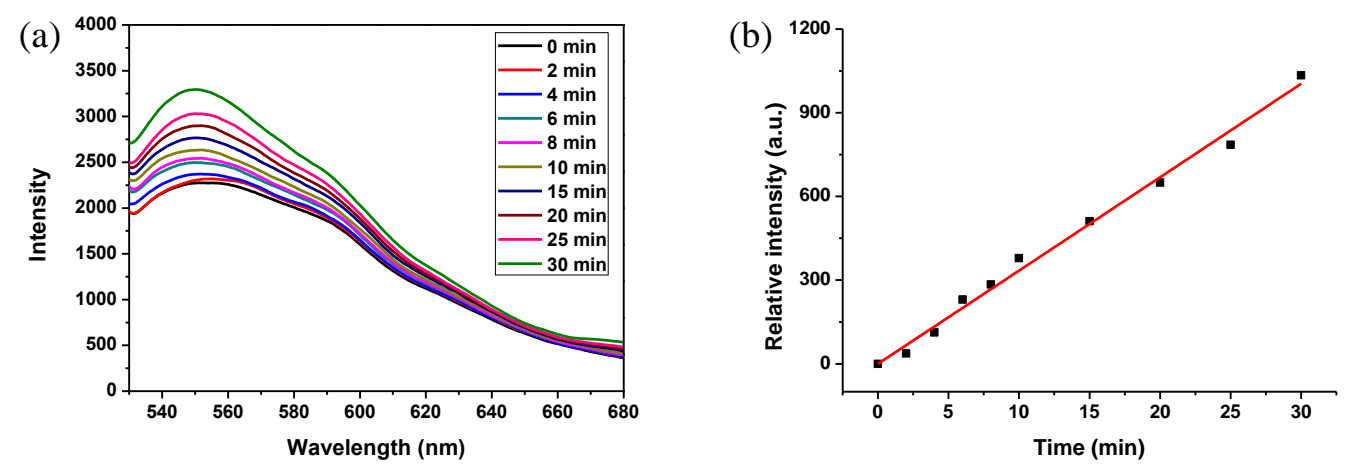

Figure S32. (a) Emission spectrum of SOSG in the presence of PG1 as a function of irradiation time $\left(\lambda_{\mathrm{ex}}=504 \mathrm{~nm}\right.$ ). (b) Singlet oxygen sensor green relative fluorescence intensity $v s$. time curve. 

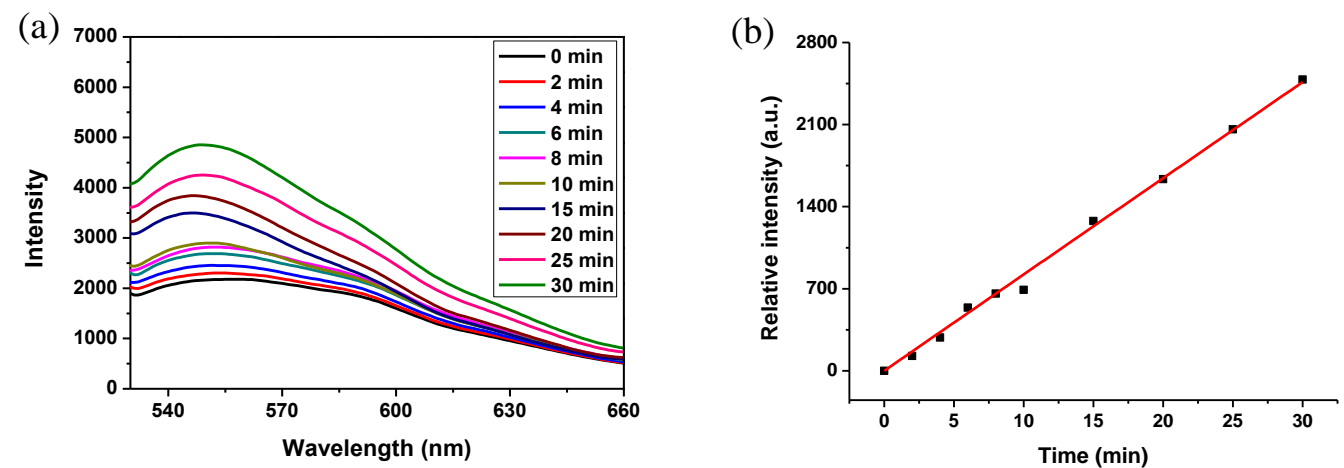

Figure S33. (a) Emission spectrum of SOSG in the presence of PG2 as a function of irradiation time $\left(\lambda_{\mathrm{ex}}=504 \mathrm{~nm}\right.$ ). (b) Singlet oxygen sensor green relative fluorescence intensity $v s$. time curve.
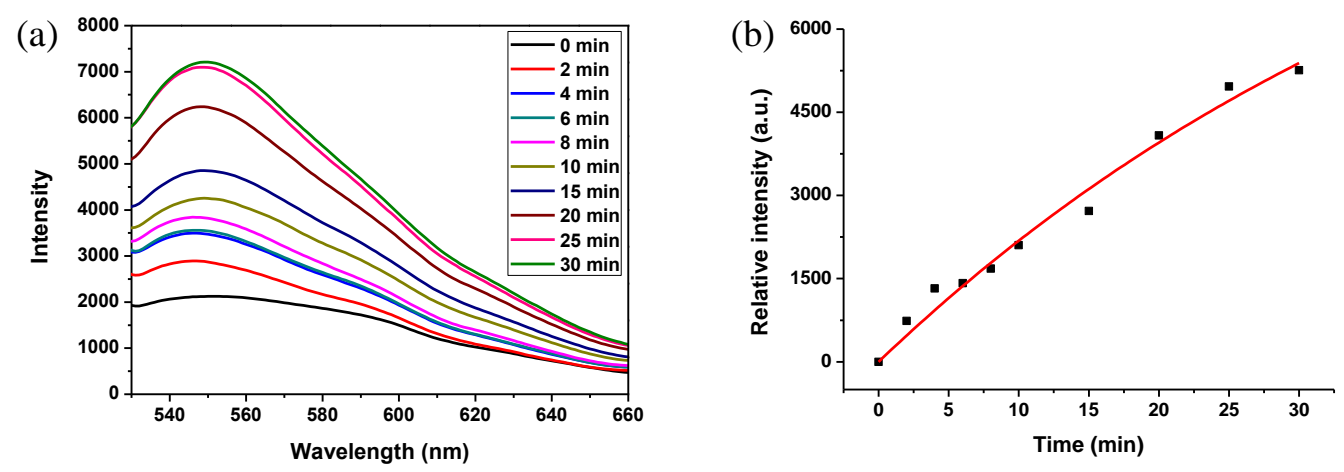

Figure S34. (a) Emission spectrum of SOSG in the presence of PG3 as a function of irradiation time $\left(\lambda_{\mathrm{ex}}=504 \mathrm{~nm}\right.$ ). (b) Singlet oxygen sensor green relative fluorescence intensity $v s$. time curve.
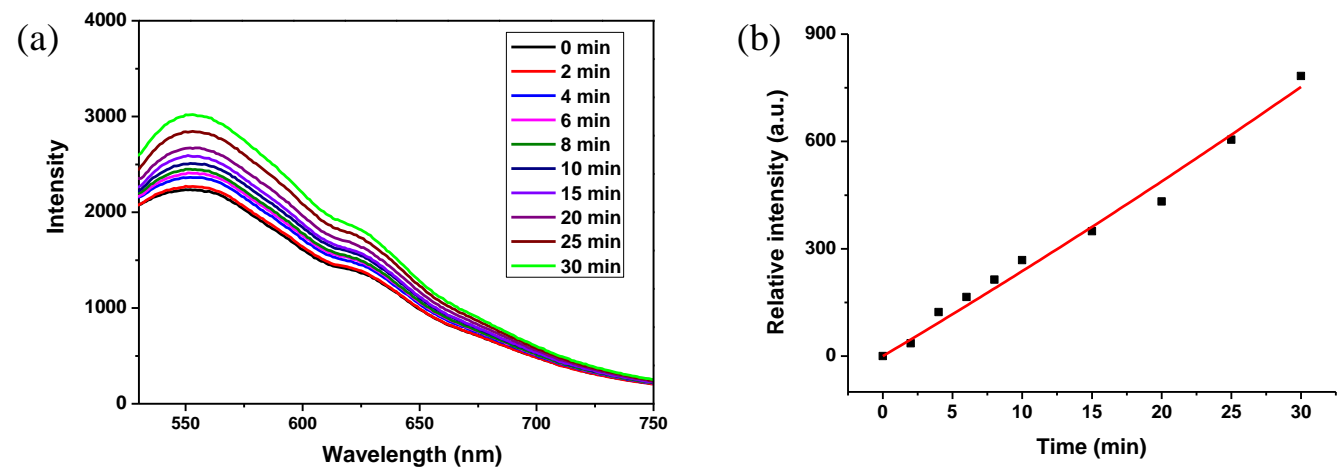

Figure S35. (a) Emission spectrum of SOSG in the presence of R-AN as a function of irradiation time $\left(\lambda_{\mathrm{ex}}=504 \mathrm{~nm}\right.$ ). (b) Singlet oxygen sensor green relative fluorescence intensity $v s$. time curve. 
(a)

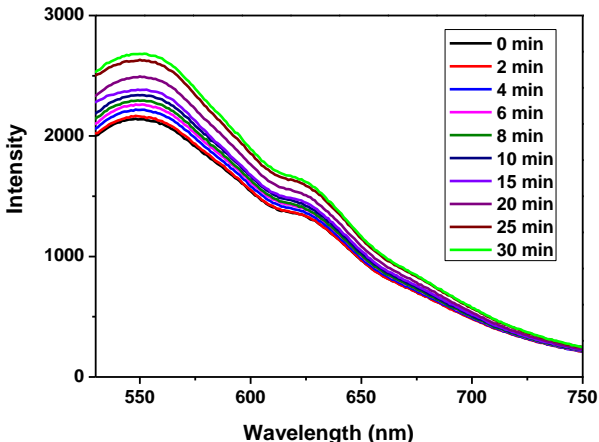

(b)

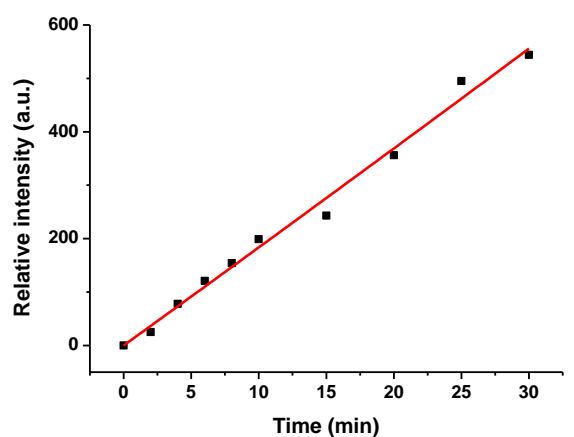

Figure S36. (a) Emission spectrum of SOSG in the presence of $\mathbf{A N}$ as a function of irradiation time $\left(\lambda_{\mathrm{ex}}=504 \mathrm{~nm}\right.$ ). (b) Singlet oxygen sensor green relative fluorescence intensity $v s$. time curve. 


\section{Section E. Mechanism study on photosensitization effect by TD-DFT}

\section{Computational details}

The ground-state structure of compound AN (169 atoms and 648 electrons) was optimized at B3LYP ${ }^{\mathrm{S} 3}-\mathrm{D} 3(\mathrm{BJ})^{\mathrm{S} 4-5} / 6-31 \mathrm{G}(\mathrm{d})^{\mathrm{S} 6-8}$ level using Gaussian 16 program $^{\mathrm{S} 9}$. And the structures of supramolecular compounds R-AN and PG1 were optimized with the GFN2-xTB semiempirical tight-binding method ${ }^{\mathrm{S} 10-11}$ using standalone $\mathrm{xtb}$ code ${ }^{\mathrm{S} 12}$ due to their significantly large molecular sizes that are unaffordable for geometry optimization with DFT method. The singlet and triplet excited states for all compounds were calculated with TDDFT method at the TD- $\omega$ B97X-D3 $313 /$ def2-SVP ${ }^{\mathrm{S} 14}$ (the effective core potential (def2-ECP) was used for the Pt element ${ }^{\mathrm{S} 15}$ ) level, and spin-orbit coupling (SOC) between singlets and triplets were computed with effective potential approach $^{\text {S16 }}$ based on the TDDFT calculations. The RIJCOSX approximation ${ }^{\text {S17-18 }}$ with $\operatorname{def} 2 / \mathrm{J}$ auxiliary basis set $^{\mathrm{S} 19}$ was used to accelerate the TDDFT calculations. To allow for the efficient subsequent TDDFT analysis, the R-AN molecule was simplified to RANM (351 atoms and 1322 electrons) and the PG1 (1029 atoms and 3882 electrons) to PG1M (519 atoms and 2352 electrons) in which the R-ANM was further simplified to R-ANM2 (181 atoms and 812 electrons). The effect of such a simplification on the calculated excited-state energies and SOC values was tested and found to have little influences (Table S7), confirming the rationality of such a simplification. All the TDDFT calculations were done with ORCA 4.2 program $^{\mathrm{S} 20-21}$, in which the "grid4" DFT integration grid and "girdx4" COSX grid with "TightSCF" convergence criteria were included. The molecular orbital (MO) distributions were rendered by VMD software $^{\mathrm{S} 22}$.

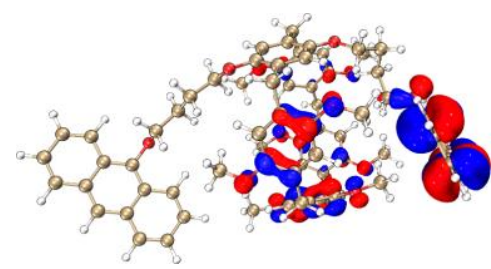

HOMO-5

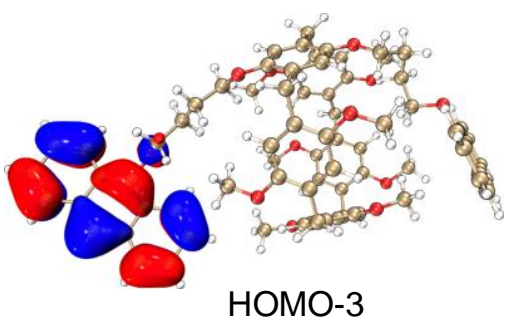

HOMO-3

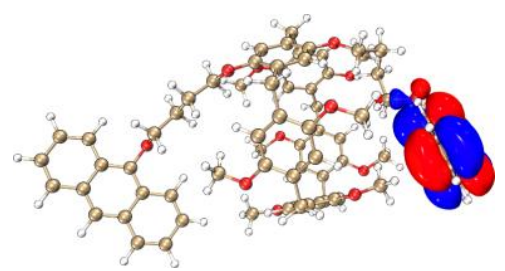

LUMO

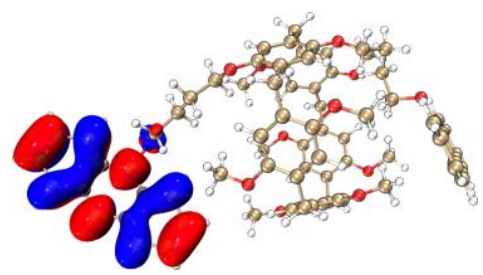

LUMO+1

Figure S37. HOMO-LUMO distributions of compound $\mathbf{A N}$ at the $\omega \mathrm{B} 97 \mathrm{X}-\mathrm{D} 3 / \mathrm{def} 2-$ SVP level. 

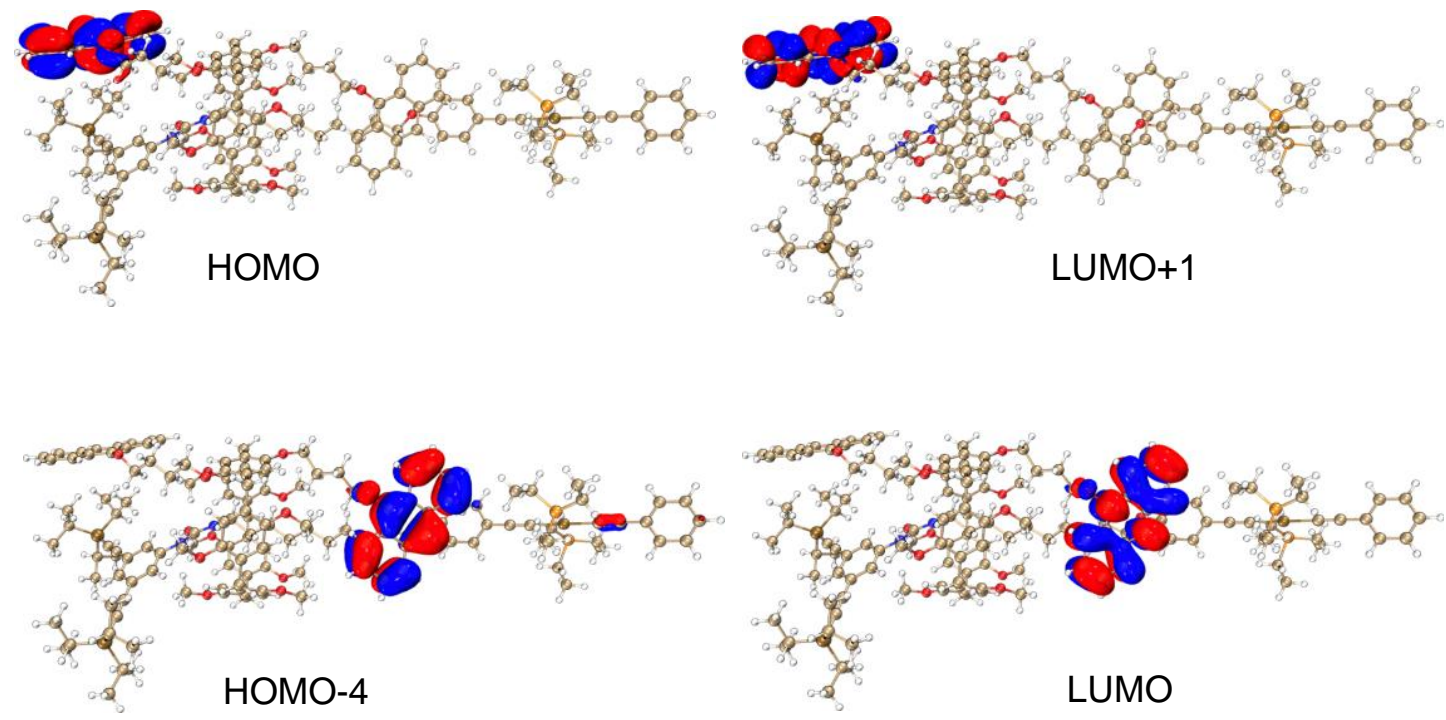

Figure S38. HOMO-LUMO distributions of compound R-ANM at the $\omega \mathrm{B} 97 \mathrm{X}-$ D3/def2-SVP level.

(a)

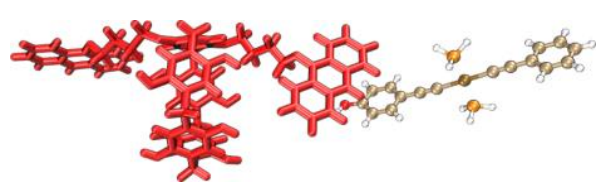

R-ANM2 (b)

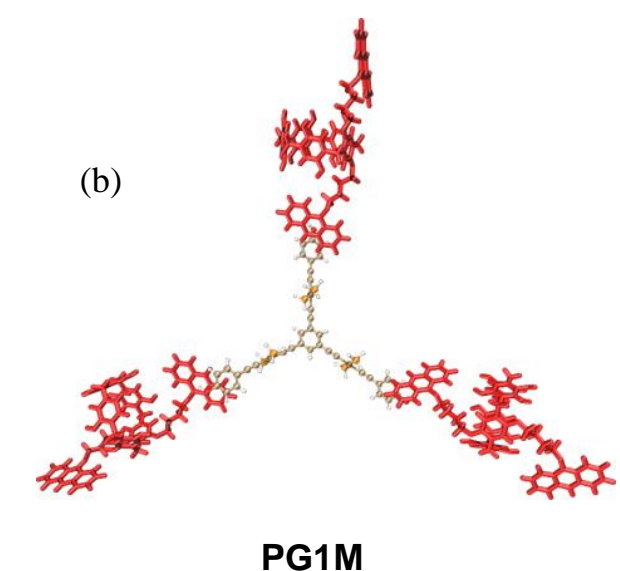

PG1M

Figure S39. Optimized structures of the effective simplified models R-ANM2 and PG1M using xtb method. 


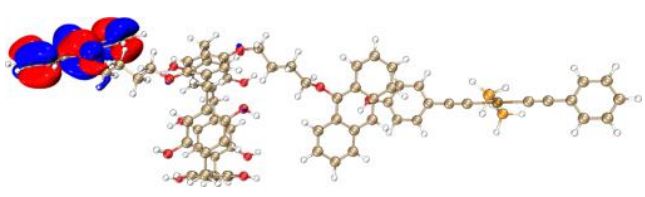

HOMO-1

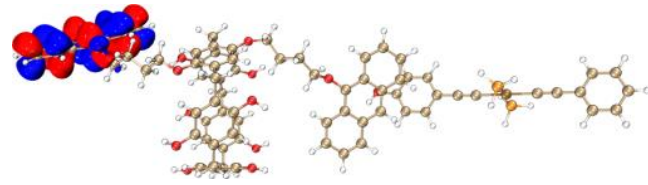

$\mathrm{LUMO}+1$

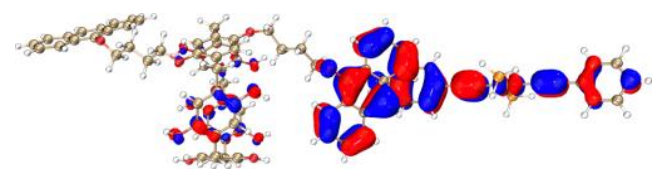

HOMO-3

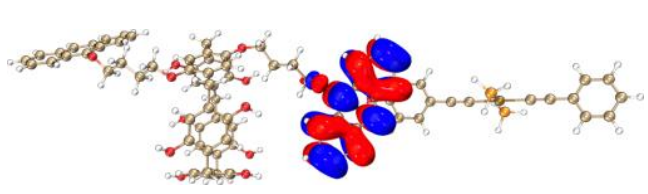

LUMO

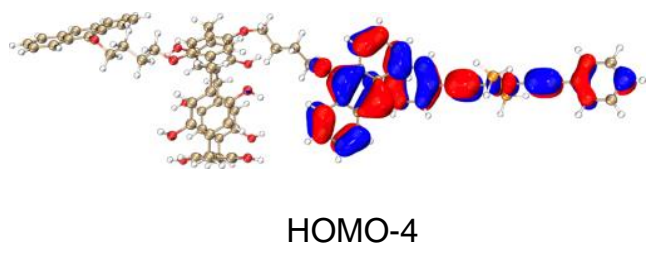

Figure S40. HOMO-LUMO distributions of compound R-ANM2 at the $\omega \mathrm{B} 97 \mathrm{X}-$ D3/def2-SVP level.

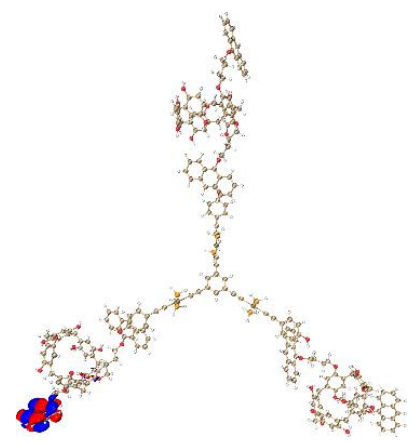

HOMO-5

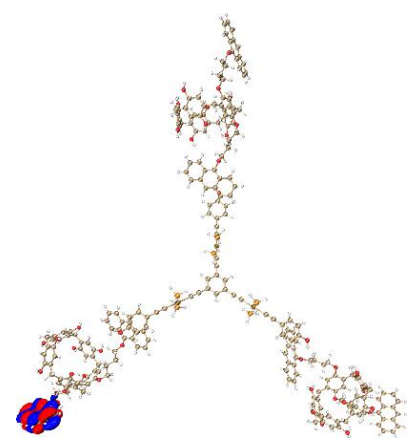

$\mathrm{LUMO}+4$

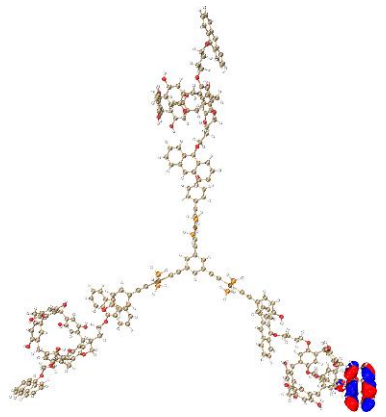

HOMO-6

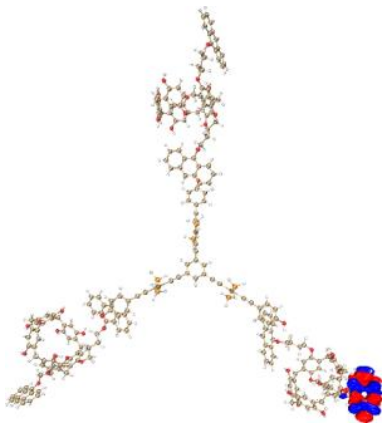

$\mathrm{LUMO}+5$

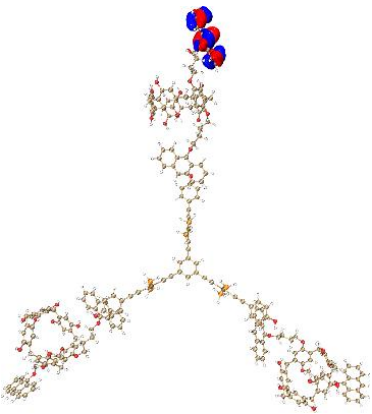

HOMO-7

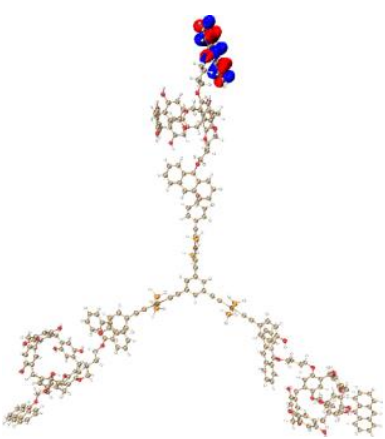

LUMO+3

Figure S41. HOMO-LUMO distributions of compound PG1M at the $\omega$ B97X-D3/def2SVP level. 
Table S3. The singlet and triplet excited states transition configurations of the molecule AN revealed by TD-DFT calculations at the $\omega$ B97X-D3/def2-SVP level.

\begin{tabular}{|c|c|c|c|c|c|}
\hline & $\mathrm{E} / \mathrm{eV}$ & $f$ & Transition configuration (\%) & $\mathrm{G}_{0} \mathrm{~W}_{0}-\mathrm{BSE}^{\mathrm{a}}$ & Exp $^{\mathrm{b}}$ \\
\hline $\mathrm{S}_{1}$ & 3.57 & 0.114 & HOMO-5 $\rightarrow$ LUMO (75) & 3.40 & 3.60 \\
\hline $\mathrm{S}_{2}$ & 3.60 & 0.155 & HOMO-3 $\rightarrow$ LUMO+1 (93) & & \\
\hline $\mathrm{T}_{1}$ & 1.68 & - & HOMO-3 $\rightarrow$ LUMO+1 (87) & 1.80 & 2.07 \\
\hline $\mathrm{T}_{2}$ & 1.68 & - & HOMO-5 $\rightarrow$ LUMO (69) & & \\
\hline
\end{tabular}

${ }^{a}$ For comparison, the high-level $\mathrm{G}_{0} \mathrm{~W}_{0}-\mathrm{BSE}$ values for anthracene molecule are taken from the work of Rangel et al. [Phys. Rev. B 2016, 93, 115206]. ${ }^{\mathrm{b}}$ Experimental data for anthracene molecule were taken from Refs. [J. Phys. Chem. A 2015, 119, 10575; J. Comput. Chem. 2017, 38, 569] and references therein.

Table S4. Spin-orbit coupling (SOC) values $\left(\mathrm{cm}^{-1}\right)$ and the energy gaps $(\mathrm{eV})$ between singlet and triplet states for $\mathrm{S}_{\mathrm{n}} / \mathrm{T}_{\mathrm{n}}(\mathrm{n}=1 \sim 4)$ of $\mathbf{A N}$ and $\mathbf{R}-\mathbf{A N M}$ molecules at the $\omega B 97 X-D 3 /$ def2-SVP level.

\begin{tabular}{cccccc|cccr}
\hline \multirow{2}{*}{$\begin{array}{c}\text { No. } \\
\text { Triplet }\end{array}$} & Singlet & \multicolumn{5}{c|}{ AN } & \multicolumn{4}{c}{ R-ANM } \\
\cline { 2 - 9 } & ES & \multicolumn{1}{c}{$\Delta$ EST } & SOC & ET & ES & \multicolumn{1}{c}{$\Delta$ EST } & \multicolumn{1}{c}{ SOC } \\
\hline 1 & 0 & 1.679 & 0.000 & -1.679 & 0.7878 & 1.650 & 0.000 & -1.650 & 1.5032 \\
1 & 1 & 1.679 & 3.574 & $\mathbf{1 . 8 9 5}$ & $\mathbf{0 . 0 1 4}$ & 1.650 & 3.519 & $\mathbf{1 . 8 6 9}$ & $\mathbf{0 . 2 9 8}$ \\
1 & 2 & 1.679 & 3.599 & $\mathbf{1 . 9 2 0}$ & $\mathbf{0 . 0 9 9}$ & 1.650 & 3.556 & $\mathbf{1 . 9 0 6}$ & $\mathbf{0 . 0 2 2}$ \\
1 & 3 & 1.679 & 4.063 & 2.384 & 0.014 & 1.650 & 4.090 & 2.440 & 2.323 \\
1 & 4 & 1.679 & 4.069 & 2.390 & 2.360 & 1.650 & 4.105 & 2.455 & 0.000 \\
2 & 0 & 1.684 & 0.000 & -1.684 & 1.4973 & 1.683 & 0.000 & -1.683 & 1.0133 \\
2 & 1 & 1.684 & 3.574 & $\mathbf{1 . 8 9 0}$ & $\mathbf{0 . 0 4 6}$ & 1.683 & 3.519 & $\mathbf{1 . 8 3 6}$ & $\mathbf{0 . 0 0 0}$ \\
2 & 2 & 1.684 & 3.599 & $\mathbf{1 . 9 1 5}$ & $\mathbf{0 . 0 0 0}$ & 1.683 & 3.556 & $\mathbf{1 . 8 7 3}$ & $\mathbf{0 . 1 4 3}$ \\
2 & 3 & 1.684 & 4.063 & 2.379 & 1.957 & 1.683 & 4.090 & 2.407 & 0.000 \\
2 & 4 & 1.684 & 4.069 & 2.385 & 0.010 & 1.683 & 4.105 & 2.422 & 1.962 \\
3 & 0 & 3.352 & 0.000 & -3.352 & 1.5287 & 3.006 & 0.000 & -3.006 & 0.6508 \\
3 & 1 & 3.352 & 3.574 & $\mathbf{0 . 2 2 2}$ & $\mathbf{0 . 0 1 7}$ & 3.006 & 3.519 & $\mathbf{0 . 5 1 3}$ & $\mathbf{0 . 0 0 0}$ \\
3 & 2 & 3.352 & 3.599 & $\mathbf{0 . 2 4 7}$ & $\mathbf{0 . 2 2 3}$ & 3.006 & 3.556 & $\mathbf{0 . 5 5 0}$ & $\mathbf{0 . 0 0 0}$ \\
3 & 3 & 3.352 & 4.063 & 0.711 & 0.000 & 3.006 & 4.090 & 1.084 & 0.000 \\
3 & 4 & 3.352 & 4.069 & 0.717 & 0.120 & 3.006 & 4.105 & 1.099 & 0.000 \\
4 & 0 & 3.354 & 0.000 & -3.354 & 1.3206 & 3.212 & 0.000 & -3.212 & 0.9361 \\
4 & 1 & 3.354 & 3.574 & $\mathbf{0 . 2 2 0}$ & $\mathbf{0 . 1 2 0}$ & 3.212 & 3.519 & $\mathbf{0 . 3 0 7}$ & $\mathbf{0 . 0 0 0}$ \\
4 & 2 & 3.354 & 3.599 & $\mathbf{0 . 2 4 5}$ & $\mathbf{0 . 0 1 4}$ & 3.212 & 3.556 & $\mathbf{0 . 3 4 4}$ & $\mathbf{0 . 0 3 3}$ \\
4 & 3 & 3.354 & 4.063 & 0.709 & 0.180 & 3.212 & 4.090 & 0.878 & 0.000 \\
4 & 4 & 3.354 & 4.069 & 0.715 & 0.000 & 3.212 & 4.105 & 0.893 & 0.030 \\
\hline & & & & & & & & &
\end{tabular}

Table S5. Spin-orbit coupling (SOC) values $\left(\mathrm{cm}^{-1}\right)$ and the energy gaps $(\mathrm{eV})$ between 
singlet and triplet states for $S_{n} / T_{n}(n=1 \sim 5)$ of PG1M molecule at the $\omega B$ 97X-D3/def2SVP level. Note that only five states were computed to improve the computational efficiency.

\begin{tabular}{cccccc}
\hline & & \multicolumn{4}{c}{ PG1M } \\
\cline { 2 - 6 } No. Triplet No. Singlet & ET & Es & $\Delta$ EsT & SOC \\
\hline 1 & 0 & 1.655 & 0.000 & -1.655 & 0.7716 \\
1 & 1 & 1.655 & 3.550 & $\mathbf{1 . 8 9 5}$ & $\mathbf{0 . 2 7 5}$ \\
1 & 2 & 1.655 & 3.554 & 1.899 & 0.000 \\
1 & 3 & 1.655 & 3.554 & 1.899 & 0.000 \\
1 & 4 & 1.655 & 3.577 & 1.922 & 0.000 \\
1 & 5 & 1.655 & 3.579 & 1.924 & 0.017 \\
2 & 0 & 1.663 & 0.000 & -1.663 & 0.7721 \\
2 & 1 & 1.663 & 3.550 & 1.887 & 0.000 \\
2 & 2 & 1.663 & 3.554 & $\mathbf{1 . 8 9 1}$ & $\mathbf{0 . 2 8 6}$ \\
2 & 3 & 1.663 & 3.554 & 1.891 & 0.061 \\
2 & 4 & 1.663 & 3.577 & 1.914 & 0.033 \\
2 & 5 & 1.663 & 3.579 & 1.916 & 0.000 \\
3 & 0 & 1.680 & 0.000 & -1.680 & 0.606 \\
3 & 1 & 1.680 & 3.550 & 1.870 & 0.000 \\
3 & 2 & 1.680 & 3.554 & 1.874 & 0.010 \\
3 & 3 & 1.680 & 3.554 & 1.874 & 0.000 \\
3 & 4 & 1.680 & 3.577 & $\mathbf{1 . 8 9 7}$ & $\mathbf{0 . 1 2 7}$ \\
3 & 5 & 1.680 & 3.579 & 1.899 & 0.010 \\
4 & 0 & 1.684 & 0.000 & -1.684 & 0.679 \\
4 & 1 & 1.684 & 3.550 & 1.866 & 0.000 \\
4 & 2 & 1.684 & 3.554 & 1.870 & 0.000 \\
4 & 3 & 1.684 & 3.554 & 1.870 & 0.017 \\
4 & 4 & 1.684 & 3.577 & 1.893 & 0.030 \\
4 & 5 & 1.684 & 3.579 & $\mathbf{1 . 8 9 5}$ & $\mathbf{0 . 1 1 7}$ \\
5 & 0 & 1.689 & 0.000 & -1.689 & 0.546 \\
5 & 1 & 1.689 & 3.550 & 1.861 & 0.014 \\
5 & 2 & 1.689 & 3.554 & 1.865 & 0.000 \\
5 & 3 & 1.689 & 3.554 & 1.865 & 0.000 \\
5 & 4 & 1.689 & 3.577 & 1.888 & 0.017 \\
5 & 5 & 1.689 & 3.579 & $\mathbf{1 . 8 9 0}$ & $\mathbf{0 . 0 6 0}$ \\
\hline & & & & &
\end{tabular}

Table S6. The singlet and triplet excited states transition configurations of the model 
compound R-ANM revealed by TD-DFT calculations at the $\omega$ B97X-D3/def2-SVP level.

\begin{tabular}{|c|c|c|c|}
\hline & E/eV & $f$ & Transition configuration (\%) \\
\hline $\mathrm{S}_{1}$ & 3.52 & 0.133 & HOMO $\rightarrow$ LUMO+1 (97) \\
\hline $\mathrm{S}_{2}$ & 3.56 & 0.193 & HOMO-4 $\rightarrow$ LUMO (94) \\
\hline $\mathrm{T}_{1}$ & 1.65 & - & HOMO $\rightarrow$ LUMO+1 (88) \\
\hline $\mathrm{T}_{2}$ & 1.68 & - & HOMO-4 $\rightarrow$ LUMO (85) \\
\hline
\end{tabular}

Table S7. The singlet and triplet excited states transition configurations of the model compound R-ANM2 revealed by TD-DFT calculations at the $\omega$ B97X-D3/def2-SVP level.

\begin{tabular}{|c|c|c|c|}
\hline & E/eV & $f$ & Transition configuration (\%) \\
\hline $\mathrm{S}_{1}$ & 3.56 & 0.173 & HOMO-1 $\rightarrow$ LUMO+1 (93) \\
\hline $\mathrm{S}_{2}$ & 3.58 & 0.182 & $\begin{array}{l}\text { HOMO-4 } \rightarrow \text { LUMO (46) } \\
\text { HOMO-3 } \rightarrow \text { LUMO (41) }\end{array}$ \\
\hline $\mathrm{T}_{1}$ & 1.66 & - & HOMO-1 $\rightarrow$ LUMO+1 (83) \\
\hline $\mathrm{T}_{2}$ & 1.69 & - & $\begin{array}{l}\text { HOMO-4 } \rightarrow \text { LUMO (42) } \\
\text { HOMO-3 } \rightarrow \text { LUMO (37) }\end{array}$ \\
\hline
\end{tabular}

Table S8. The singlet and triplet excited states transition configurations of the model compound PG1M revealed by TD-DFT calculations at the $\omega$ B97X-D3/def2-SVP level.

\begin{tabular}{|c|c|c|c|}
\hline & E/eV & $f$ & Transition configuration (\%) \\
\hline $\mathrm{S}_{1}$ & 3.55 & 0.183 & HOMO-5 $\rightarrow$ LUMO+4 (90) \\
\hline $\mathrm{S}_{2}$ & 3.55 & 0.164 & HOMO-6 $\rightarrow$ LUMO+5 (90) \\
\hline $\mathrm{S}_{3}$ & 3.55 & 0.178 & HOMO-7 $\rightarrow$ LUMO+3 (90) \\
\hline $\mathrm{T}_{1}$ & 1.66 & - & HOMO-5 $\rightarrow$ LUMO+4 (82) \\
\hline $\mathrm{T}_{2}$ & 1.66 & - & HOMO-6 $\rightarrow$ LUMO+5 (86) \\
\hline $\mathrm{T}_{3}$ & 1.68 & - & HOMO-7 $\rightarrow$ LUMO+3 (62) \\
\hline
\end{tabular}

Section F. Photolysis of the rotaxane-branched dendrimers 
(a)

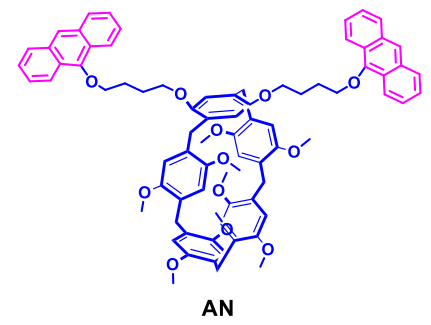

(c)

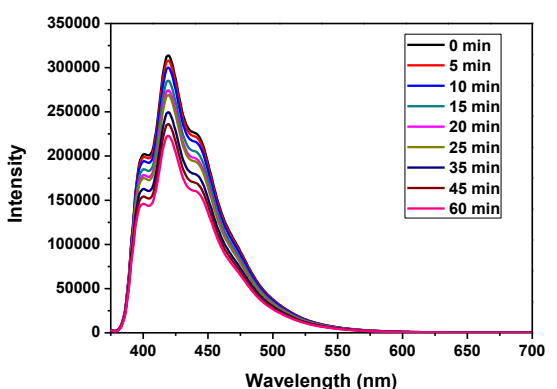

(b)

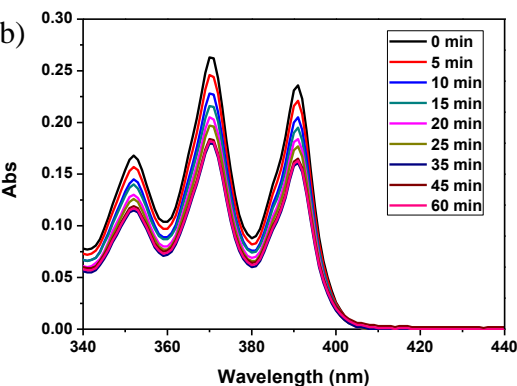

(d)

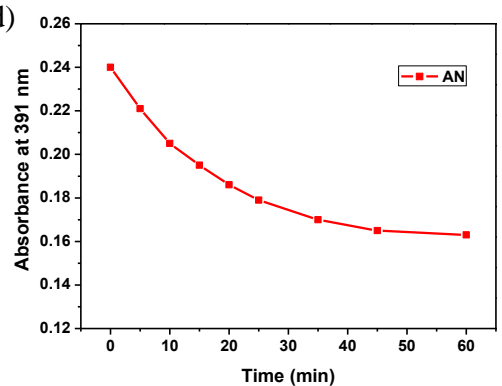

Figure S42. (a) The chemical structure of AN. (b) UV-vis spectra (0.042 mM in THF of anthracene unit), (c) fluorescence spectra $\left(\lambda_{\mathrm{ex}}=370 \mathrm{~nm}\right)$ and (d) absorbance at 391 $\mathrm{nm}$ upon irradiation at $365 \mathrm{~nm}$ for different time. Measured at $25^{\circ} \mathrm{C}$.

(a)

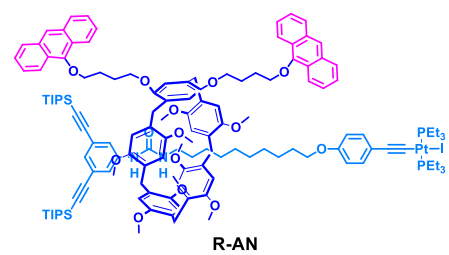

(c)

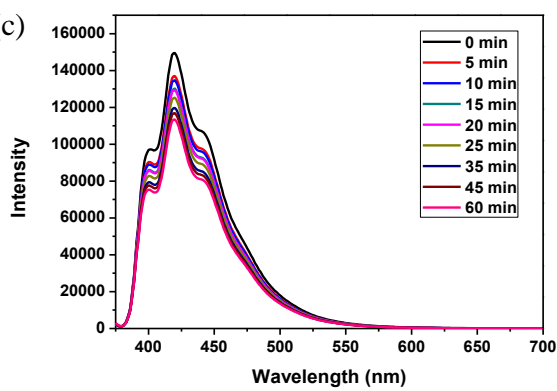

(b)

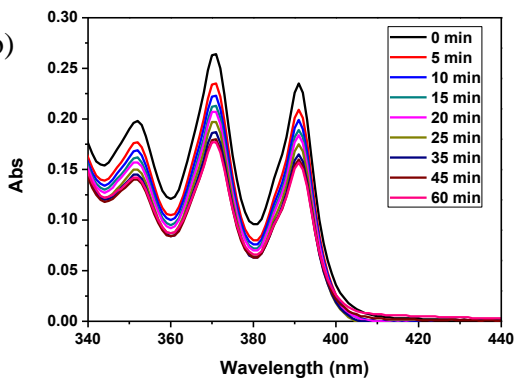

(d)

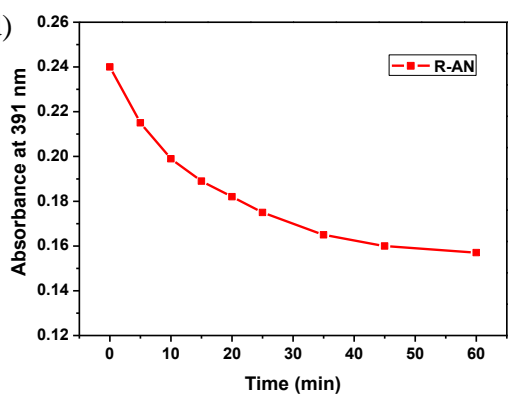

Figure S43. (a) The chemical structure of [2] rotaxane R-AN. (b) UV-vis spectra (0.042 $\mathrm{mM}$ in THF of anthracene unit), (c) fluorescence spectra $\left(\lambda_{\text {ex }}=370 \mathrm{~nm}\right)$ and (d) absorbance at $391 \mathrm{~nm}$ upon irradiation at $365 \mathrm{~nm}$ for different time. Measured at $25^{\circ} \mathrm{C}$. 
(a)

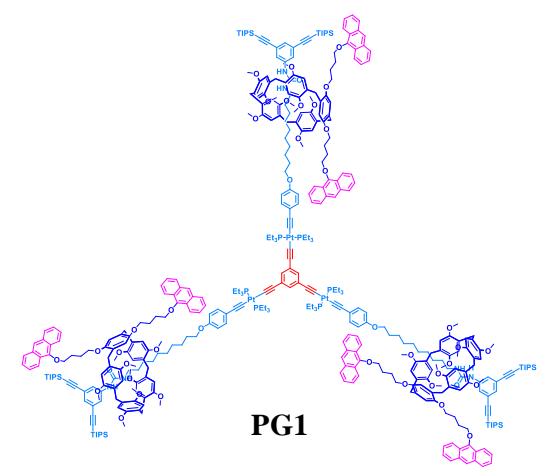

(c)

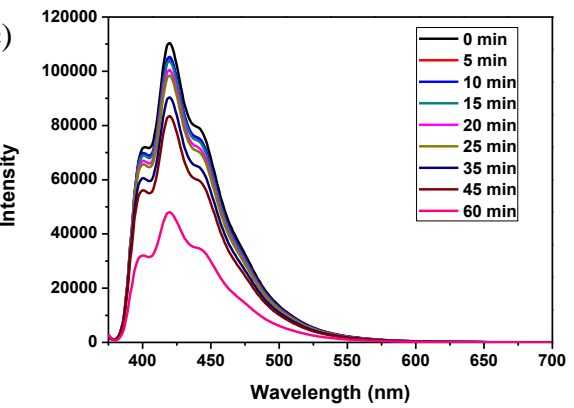

(b)

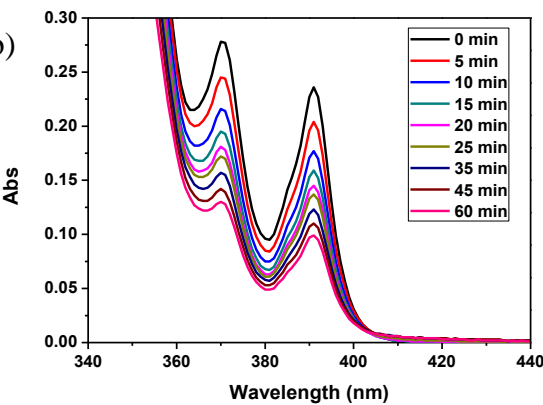

(d)

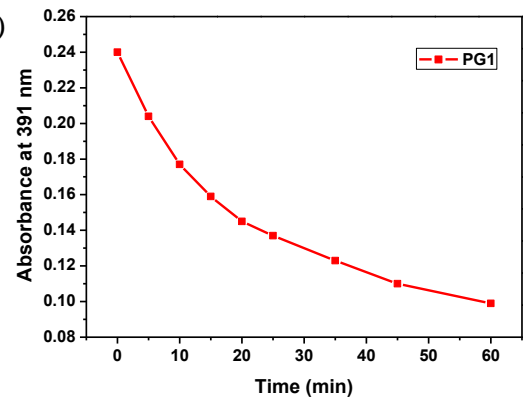

Figure S44. (a) The chemical structure of PG1. (b) UV-vis spectra (0.042 mM in THF of anthracene unit), (c) fluorescence spectra $\left(\lambda_{\mathrm{ex}}=370 \mathrm{~nm}\right)$ and $(\mathrm{d})$ absorbance at 391 $\mathrm{nm}$ upon irradiation at $365 \mathrm{~nm}$ for different time. Measured at $25^{\circ} \mathrm{C}$.

(a)
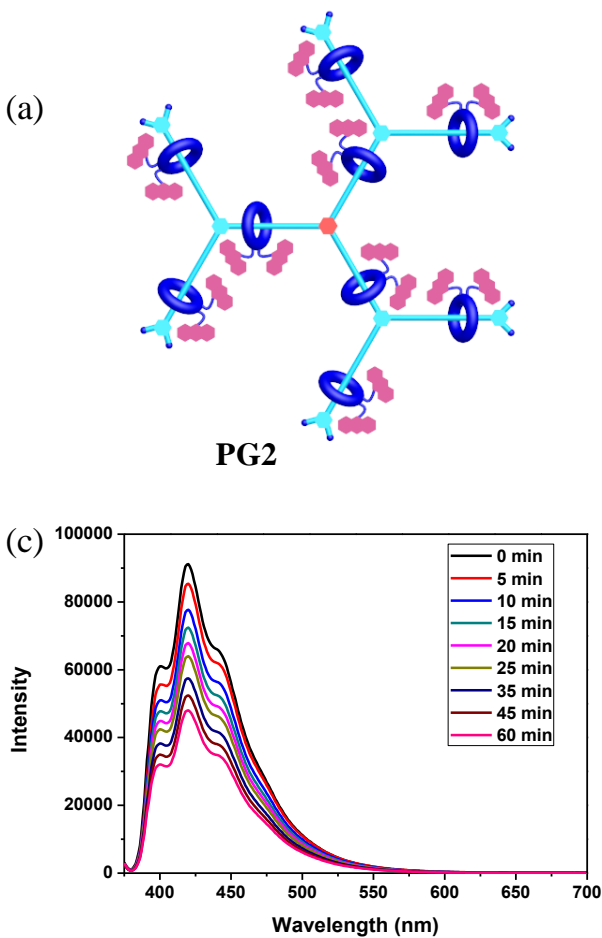

(b)

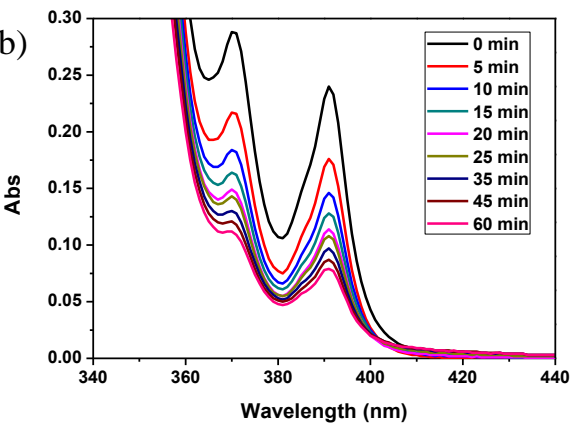

(d)

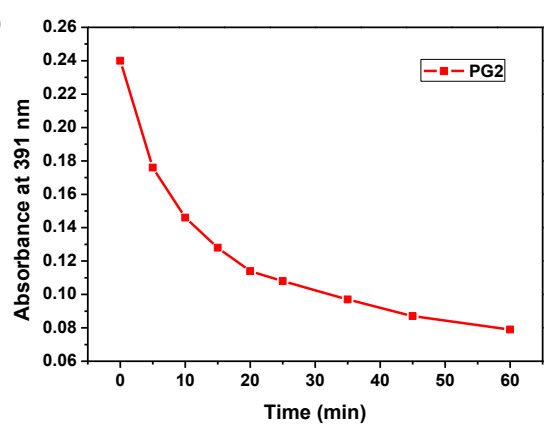

Figure S45. (a) The cartoon representation of PG2. (b) UV-vis spectra $(0.042 \mathrm{mM}$ in THF of anthracene unit), (c) fluorescence spectra $\left(\lambda_{\mathrm{ex}}=370 \mathrm{~nm}\right)$ and $(\mathrm{d})$ absorbance at $391 \mathrm{~nm}$ upon irradiation at $365 \mathrm{~nm}$ for different time. Measured at $25^{\circ} \mathrm{C}$. 
(a)
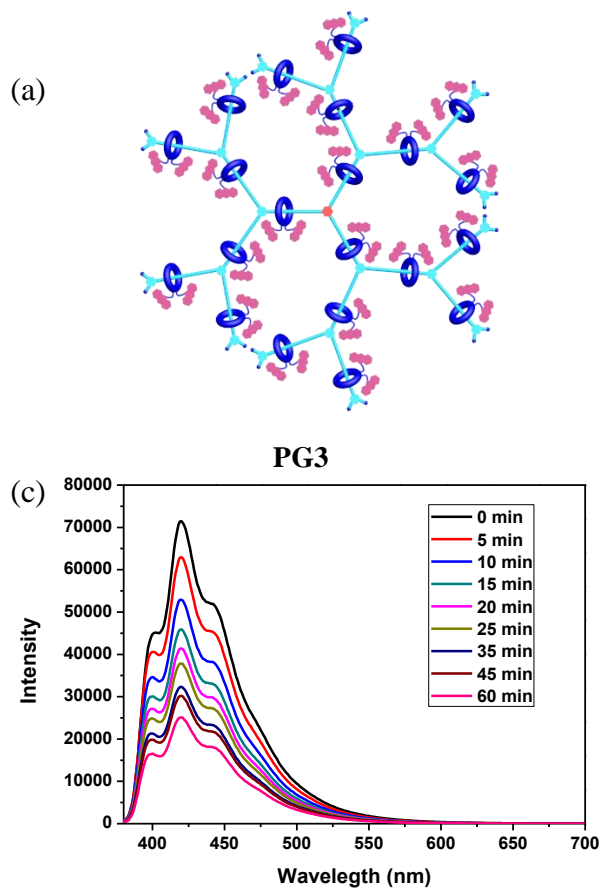

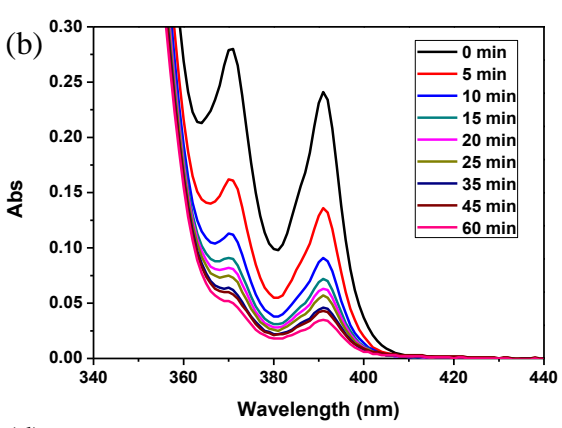

(d)

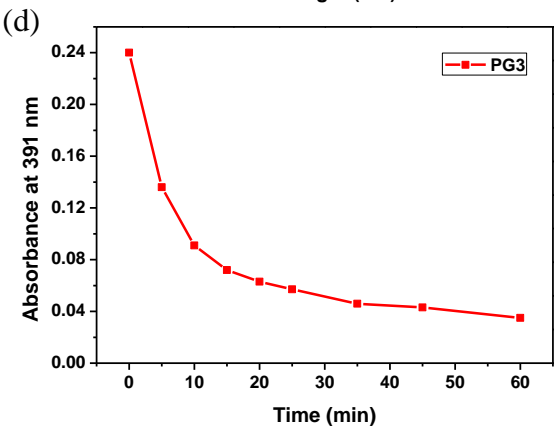

Figure S46. (a) The cartoon representation of PG3. (b) UV-vis spectra $(0.042 \mathrm{mM}$ in THF of anthracene unit), (c) fluorescence spectra $\left(\lambda_{\mathrm{ex}}=370 \mathrm{~nm}\right)$ and $(\mathrm{d})$ absorbance at $391 \mathrm{~nm}$ upon irradiation at $365 \mathrm{~nm}$ for different time. Measured at $25^{\circ} \mathrm{C}$.

(a)

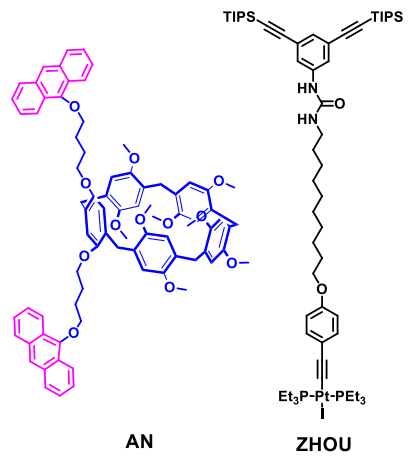

(c)

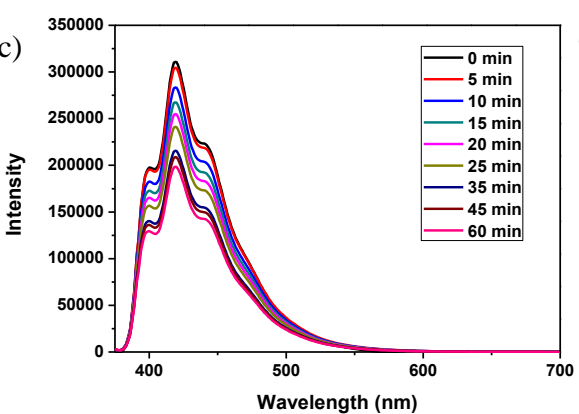

(b)
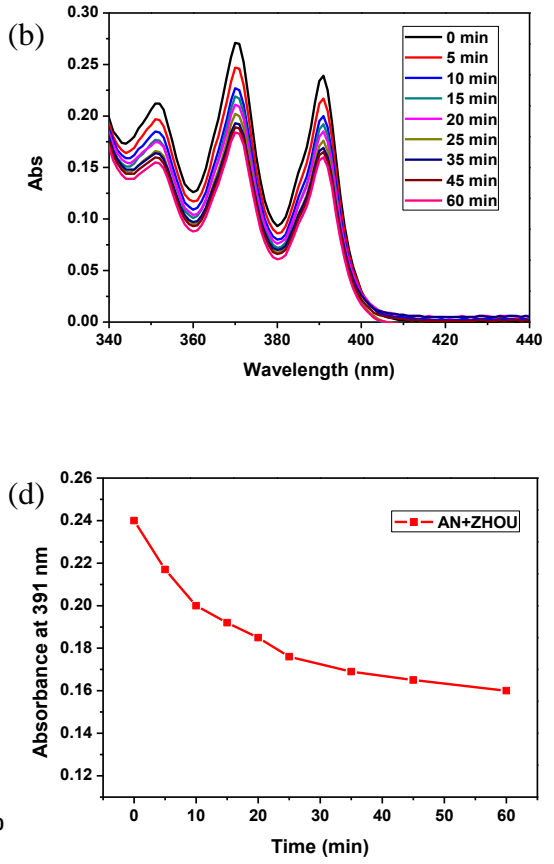

Figure S47. (a) The chemical structure of $\mathbf{A N}$ and model compound $\mathbf{Z H O U}{ }^{\mathrm{S} 2}$. (b) UVvis spectra, (c) fluorescence spectra $\left(\lambda_{\text {ex }}=370 \mathrm{~nm}\right)$ and (d) absorbance at $391 \mathrm{~nm}$ upon irradiation at $365 \mathrm{~nm}$ for different time. Measured at $25^{\circ} \mathrm{C},[\mathbf{A N}]=0.021 \mathrm{mM},[\mathbf{Z H O U}]$ $=0.021 \mathrm{mM}$. 
(a)

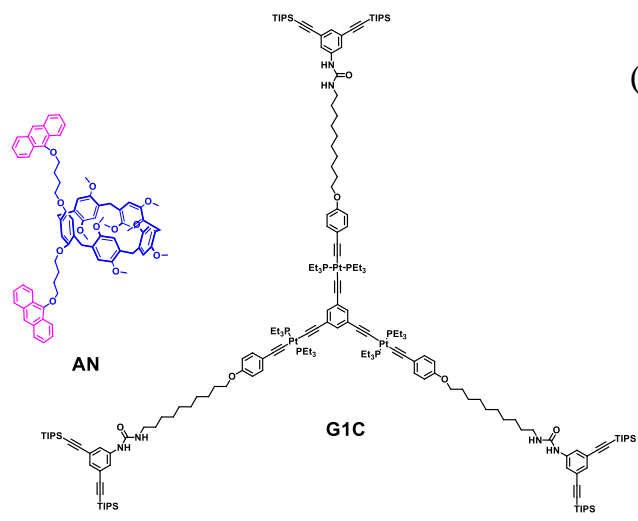

(c)

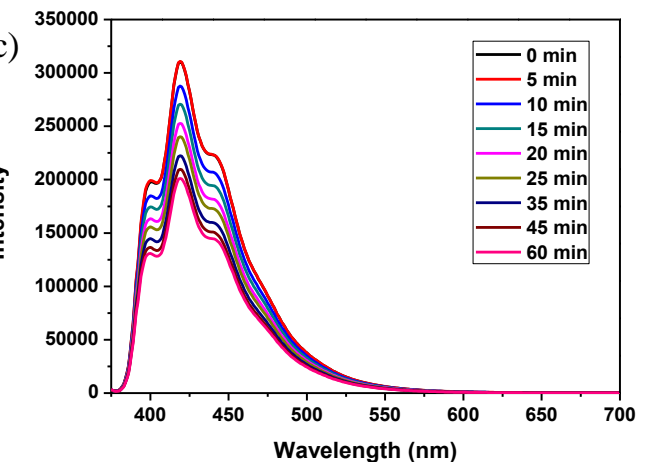

(b)

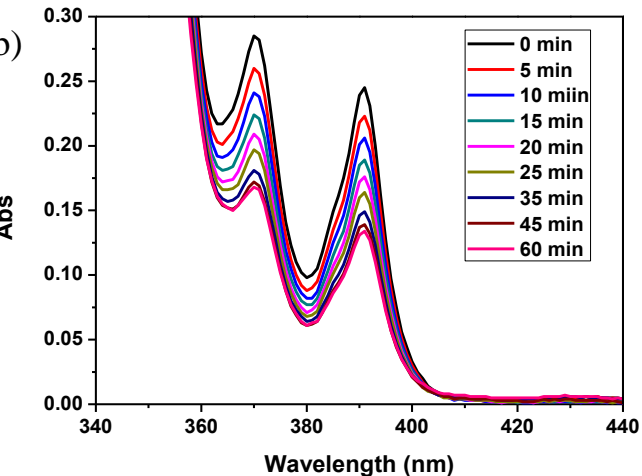

(d)

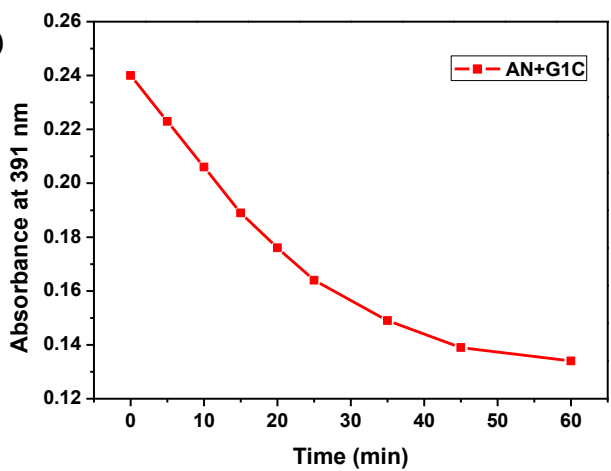

Figure S48. (a) The chemical structure of $\mathbf{A N}$ and model compound $\mathbf{G 1 C}^{\mathrm{S} 2}$. (b) UVvis spectra, (c) fluorescence spectra $\left(\lambda_{\text {ex }}=370 \mathrm{~nm}\right)$ and $(\mathrm{d})$ absorbance at $391 \mathrm{~nm}$ upon irradiation at $365 \mathrm{~nm}$ for different time. Measured at $25^{\circ} \mathrm{C},[\mathbf{A N}]=0.021 \mathrm{mM},[\mathbf{G 1 C}]$ $=0.007 \mathrm{mM}$.

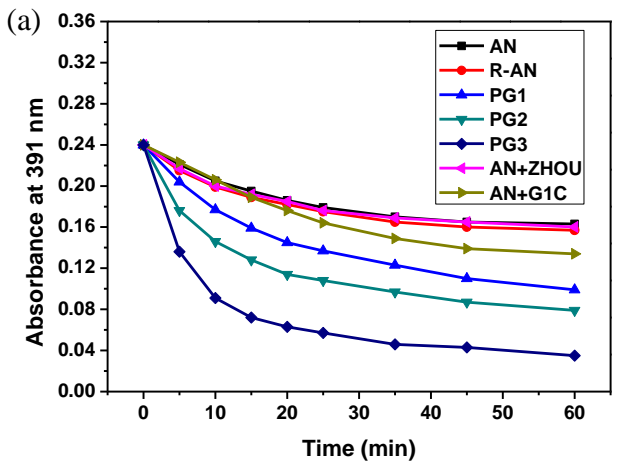

(b)

\begin{tabular}{cc}
\hline Compound & Normalized rate \\
\hline AN & 0.92 \\
\hline AN + ZHOU & 0.96 \\
\hline R-AN & 1.00 \\
\hline AN + G1C & 1.27 \\
\hline PG1 & 1.70 \\
\hline PG2 & 1.94 \\
\hline PG3 & 2.45
\end{tabular}

Figure S49. (a) Absorbance of AN, R-AN, PGn ( $\mathrm{n}=1,2,3)$ and model experiments $\mathbf{A N}+\mathbf{Z H O U}, \mathbf{A N}+\mathbf{G 1 C}$ at $391 \mathrm{~nm}$ upon irradiation at $365 \mathrm{~nm}$ for different time. Measured at $25{ }^{\circ} \mathrm{C}$. (b) Normalized photolysis rates of anthracene at $391 \mathrm{~nm}$ upon irradiation for $1 \mathrm{~h}$. 
(a)

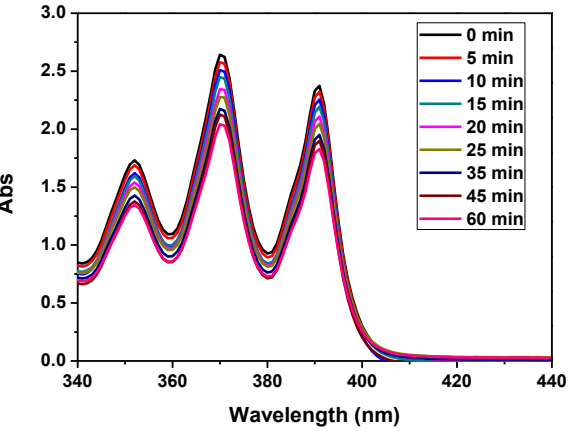

(b)

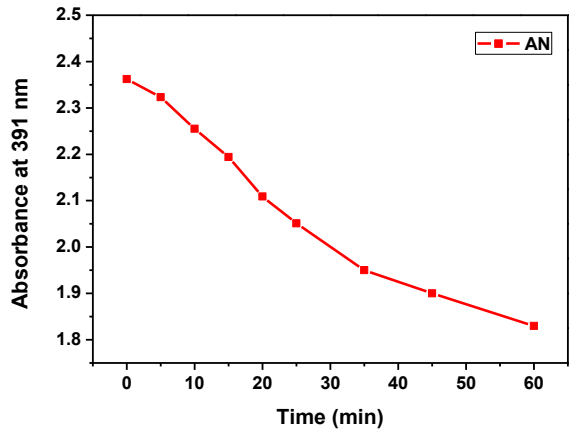

Figure S50. (a) UV-vis spectra (0.42 $\mathrm{mM}$ in THF of anthracene unit) upon irradiation and (b) absorbance of $\mathbf{A N}$ at $391 \mathrm{~nm}$ upon irradiation at $365 \mathrm{~nm}$ for different time. Measured at $25^{\circ} \mathrm{C}$.

(a)

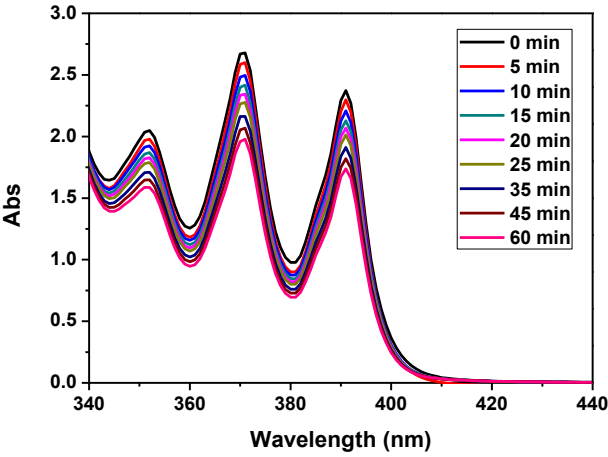

(b)

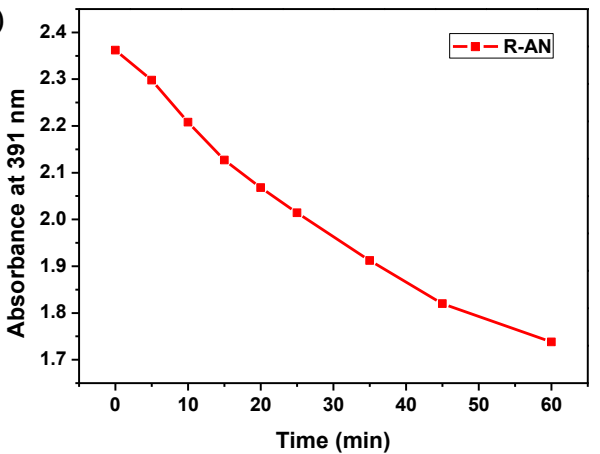

Figure S51. (a) UV-vis spectra (0.42 mM in THF of anthracene unit) and (b) absorbance of 2]rotaxane R-AN at $391 \mathrm{~nm}$ upon irradiation at $365 \mathrm{~nm}$ for different time. Measured at $25^{\circ} \mathrm{C}$.

(a)

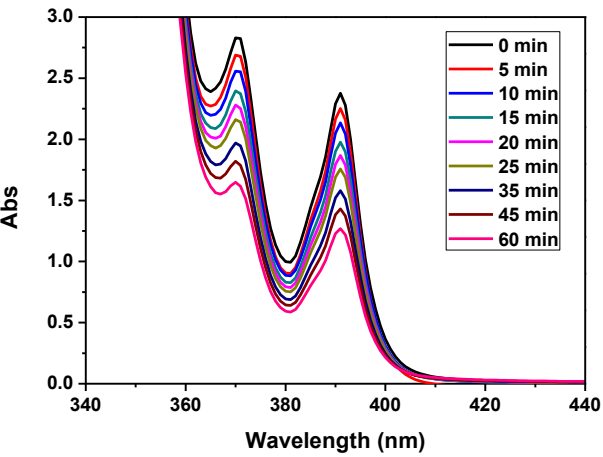

(b)

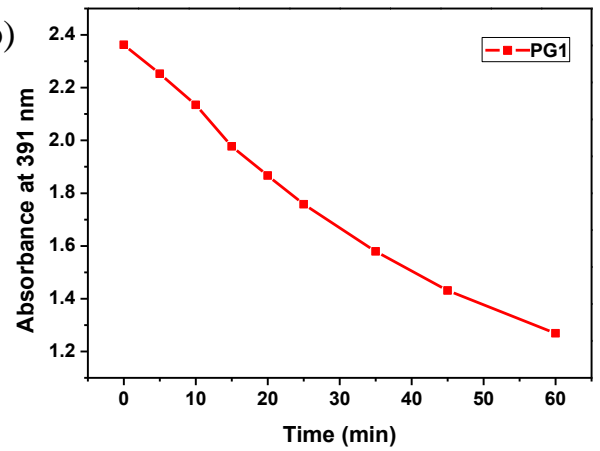

Figure S52. (a) UV-vis spectra (0.42 $\mathrm{mM}$ in THF of anthracene unit) and (b) absorbance of PG1 at $391 \mathrm{~nm}$ upon irradiation at $365 \mathrm{~nm}$ for different time. Measured at $25{ }^{\circ} \mathrm{C}$. 
(a)

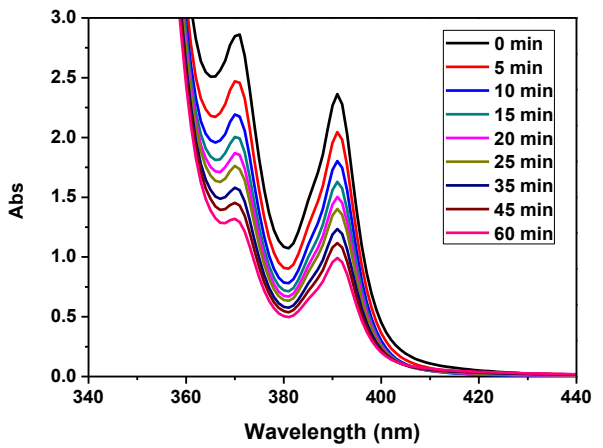

(b)

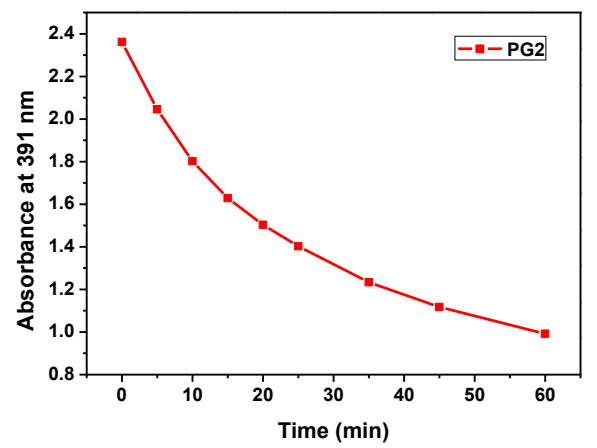

Figure S53. (a) UV-vis spectra ( $0.42 \mathrm{mM}$ in THF of anthracene unit) and (b) absorbance of PG2 at $391 \mathrm{~nm}$ upon irradiation at $365 \mathrm{~nm}$ for different time. Measured at $25^{\circ} \mathrm{C}$.

(a)

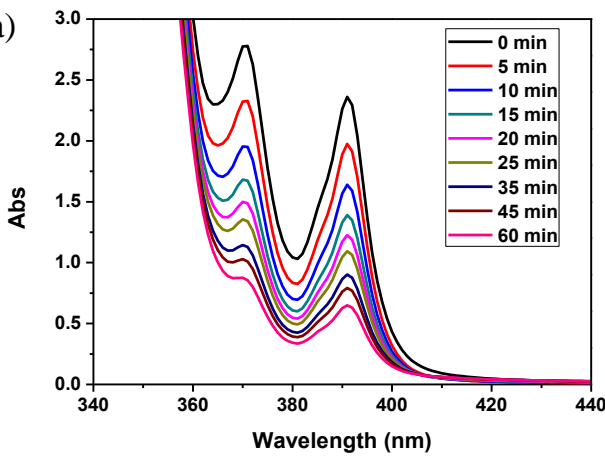

(b)

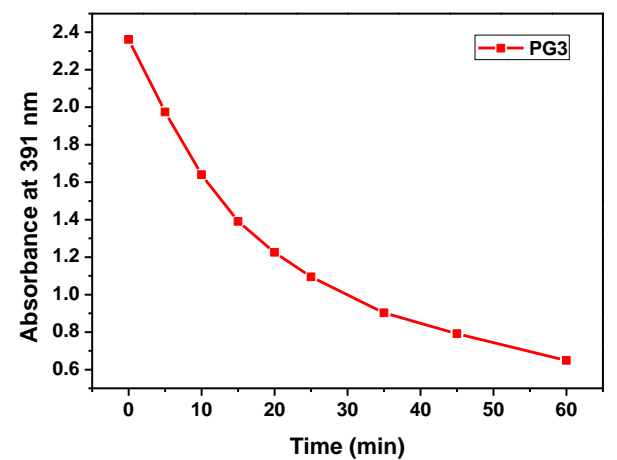

Figure S54. (a) UV-vis spectra (0.42 $\mathrm{mM}$ in THF of anthracene unit) and (b) absorbance of PG3 at $391 \mathrm{~nm}$ upon irradiation at $365 \mathrm{~nm}$ for different time. Measured at $25^{\circ} \mathrm{C}$. 
Section G. Phtooxidation mechanism of the rotaxane-branched dendrimers

Scheme S6. Proposed mechanism of photooxidation and further decomposition of 9anthroxy alkyl ether.

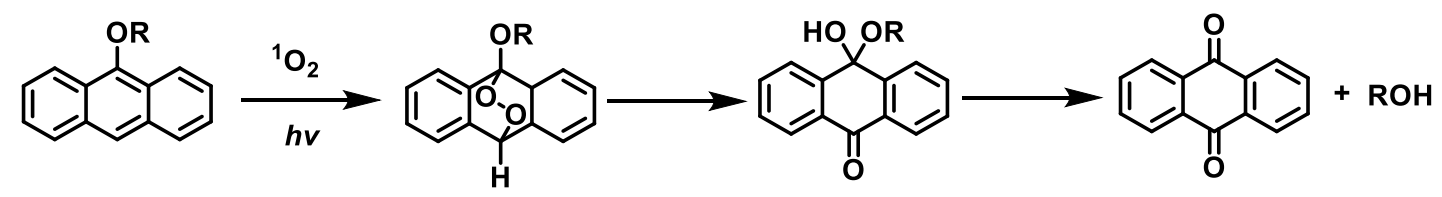

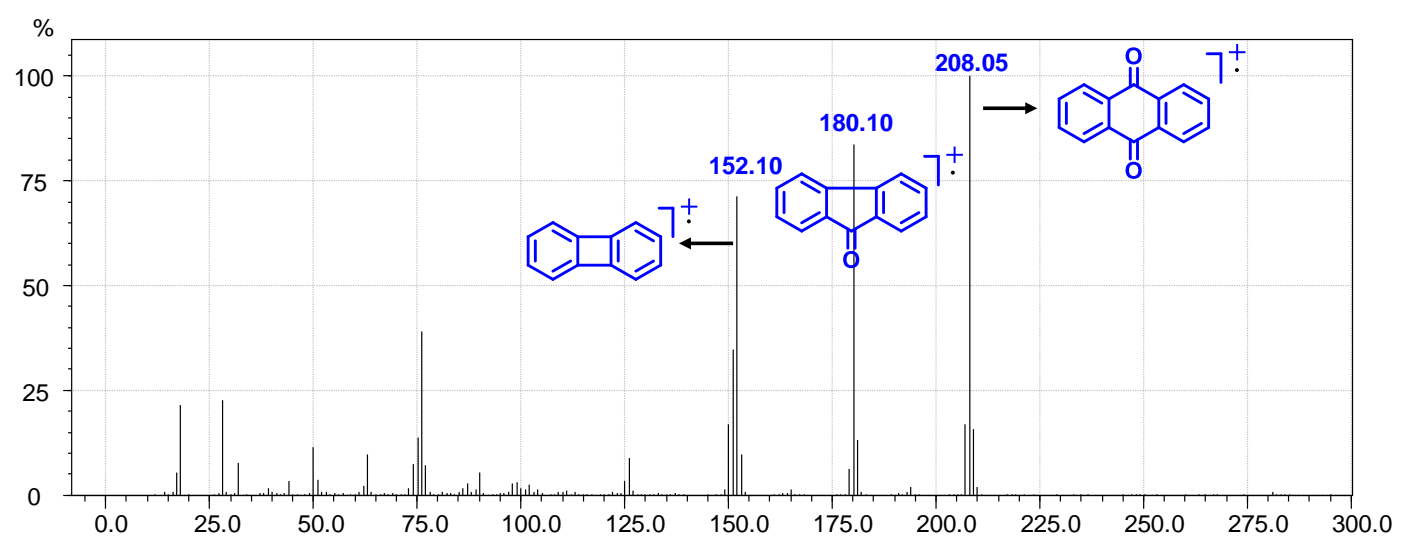

Figure S55. EI-MS spectrum of the precipitates produced in the experiments upon UV irradiation $(365 \mathrm{~nm})$.

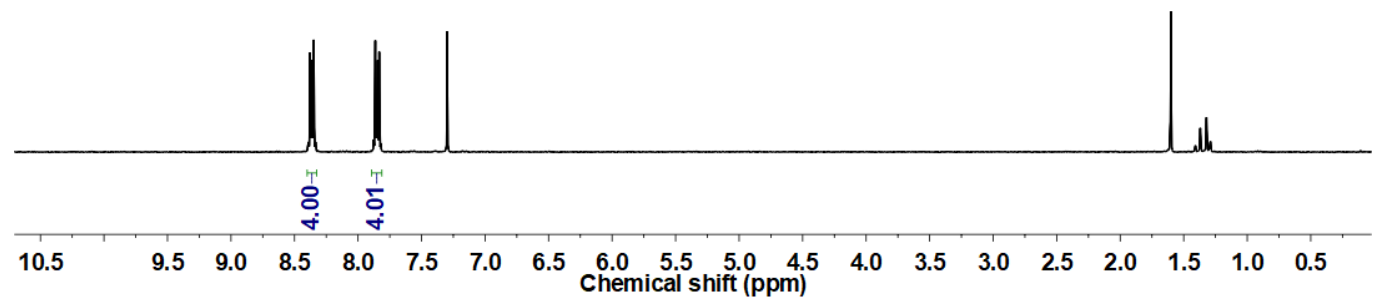

Figure S56. ${ }^{1} \mathrm{H}$ NMR spectrum $\left(\mathrm{CDCl}_{3}, 298 \mathrm{~K}, 500 \mathrm{MHz}\right)$ of the precipitates produced in the experiments upon UV irradiation $(365 \mathrm{~nm})$. 


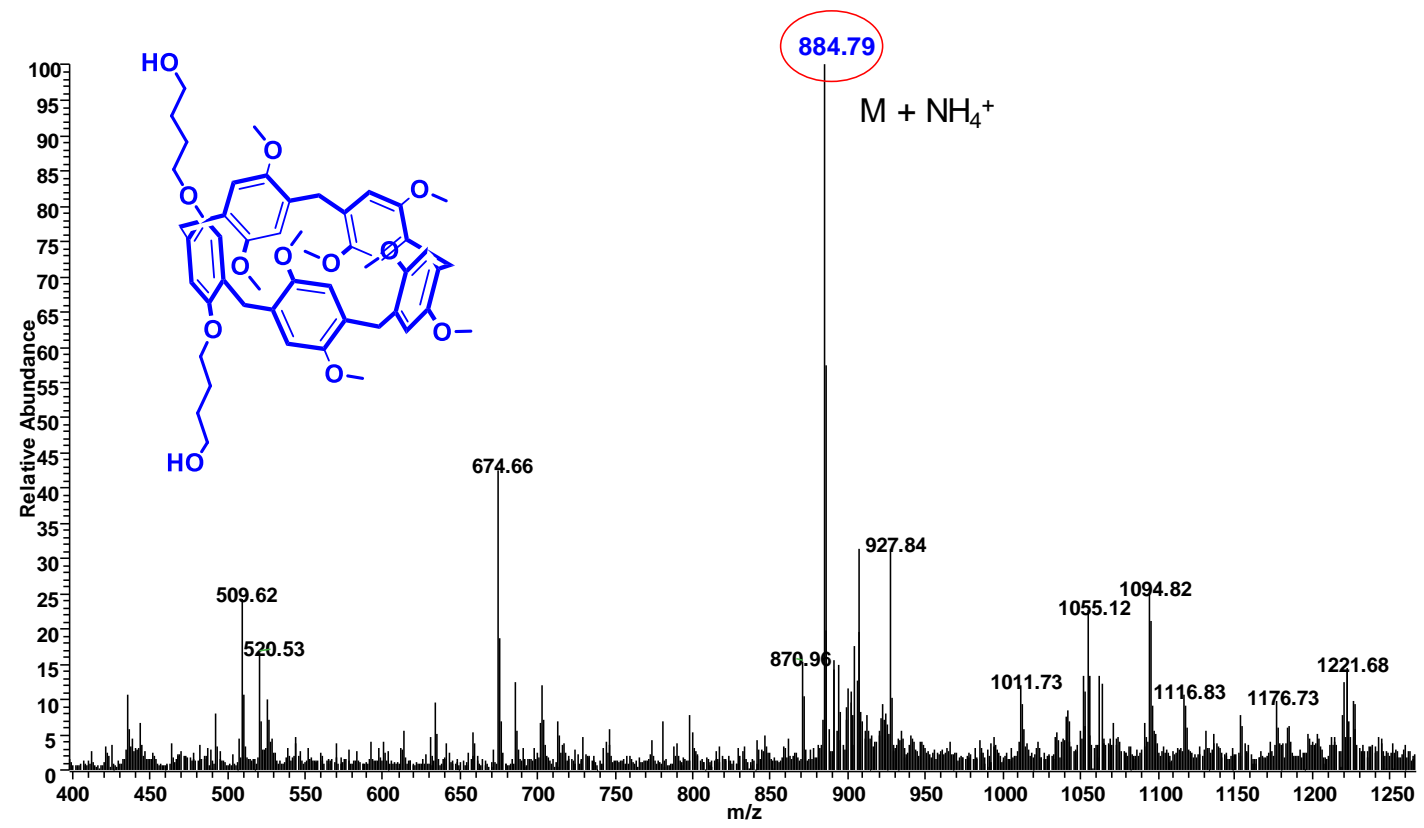

Figure S57. ESI-MS spectrum of AN after UV irradiation $(365 \mathrm{~nm})$ for $1 \mathrm{~h}$.

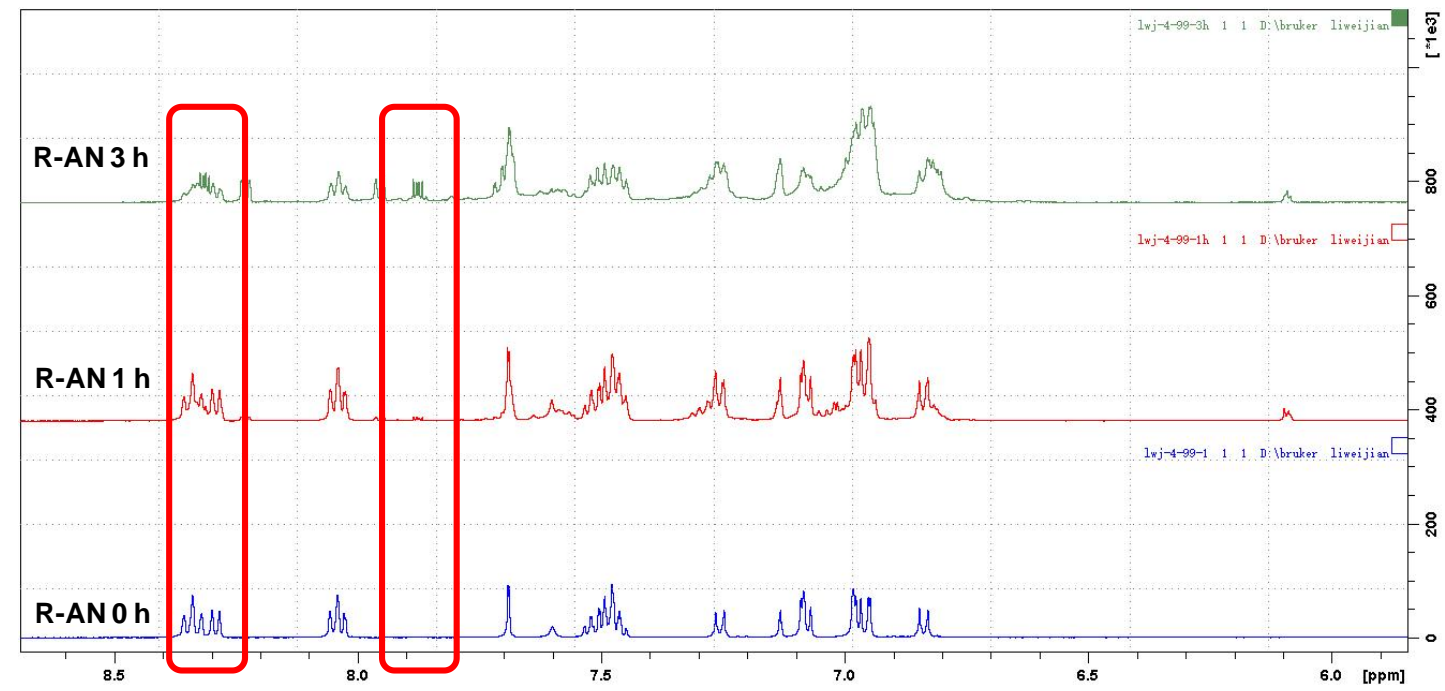

Figure S58. Partial ${ }^{1} \mathrm{H}$ NMR spectra (THF- $d_{8}, 298 \mathrm{~K}, 500 \mathrm{MHz}$ ) of [2]rotaxane R-AN upon irradiation at $365 \mathrm{~nm}$ for different time. 


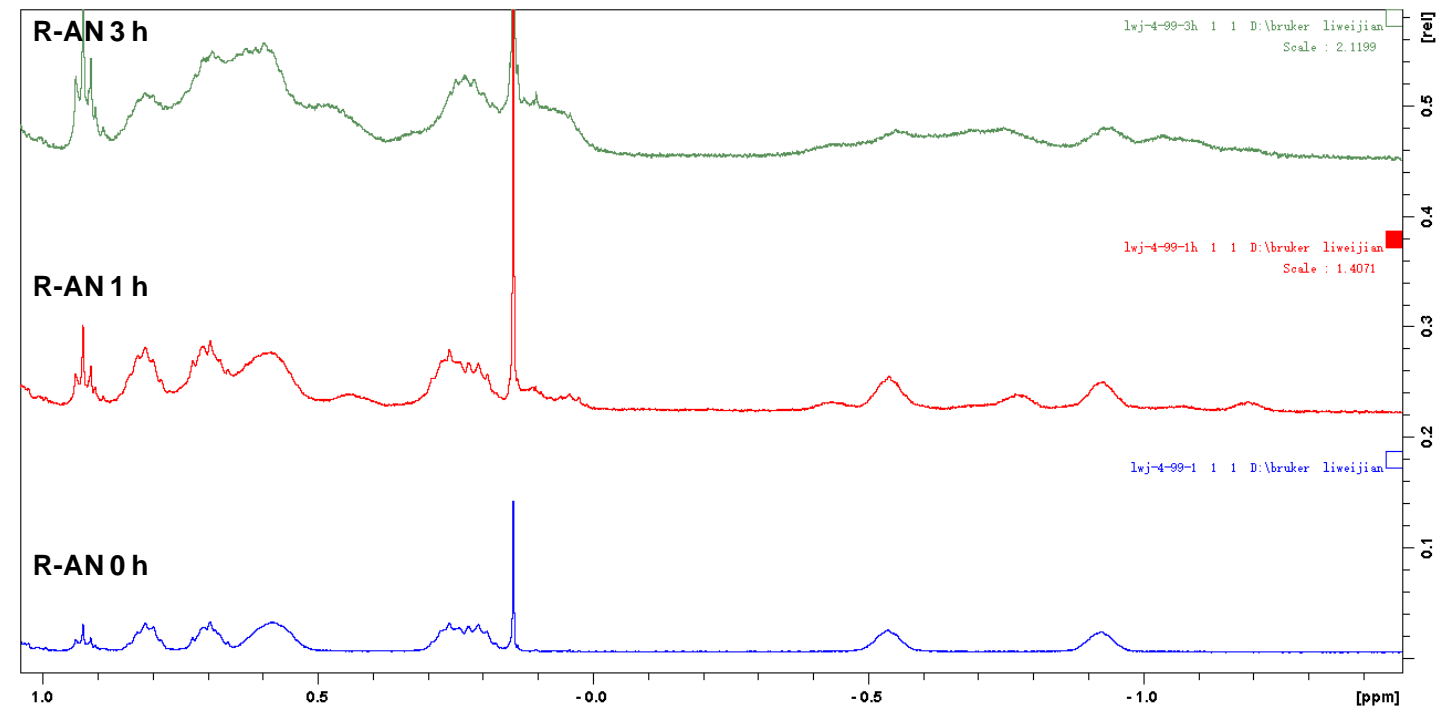

Figure S59. Partial ${ }^{1} \mathrm{H}$ NMR spectra (THF- $d_{8}, 298 \mathrm{~K}, 500 \mathrm{MHz}$ ) of [2]rotaxane R-AN upon irradiation at $365 \mathrm{~nm}$ for different time.

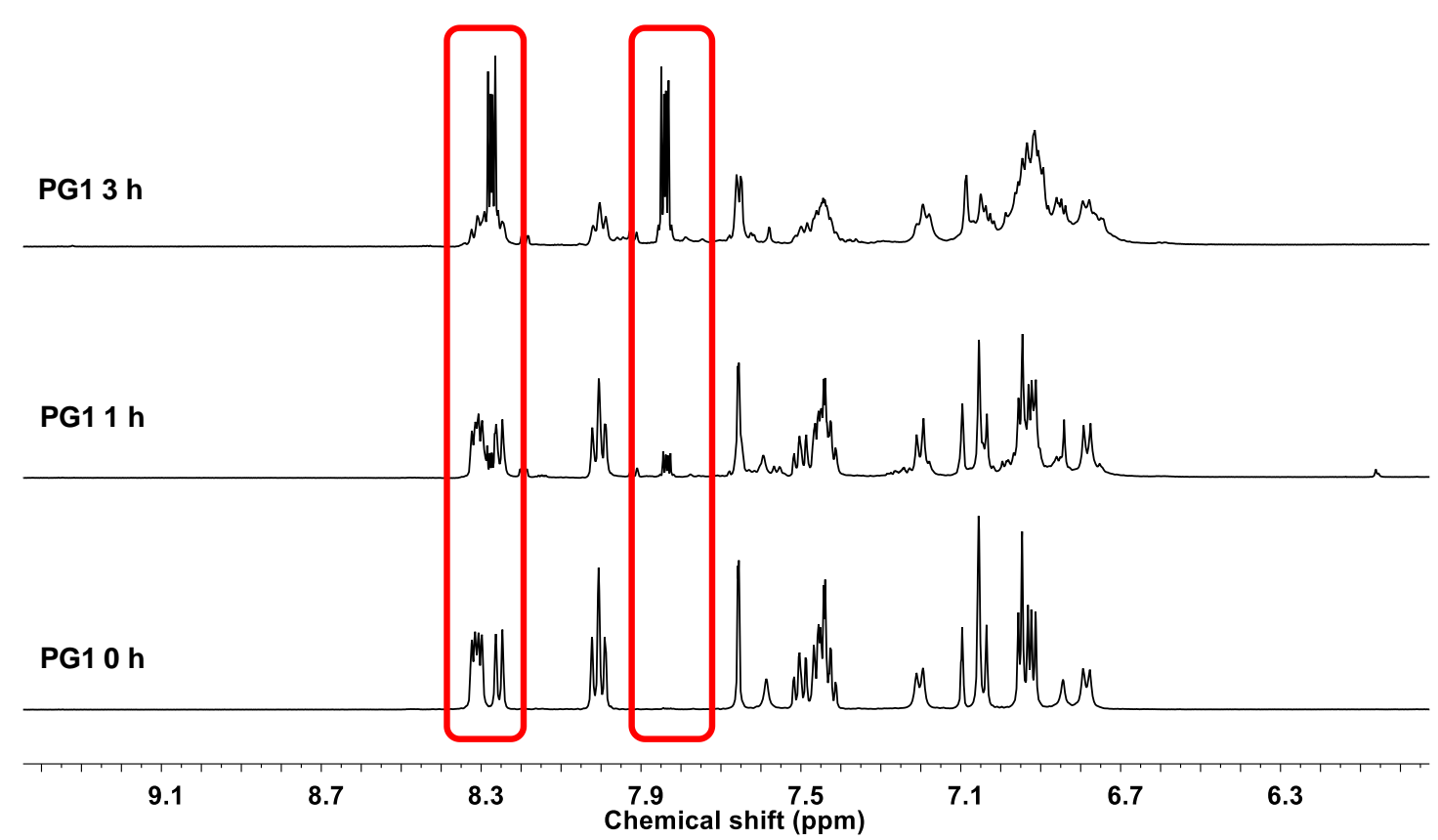

Figure S60. Partial ${ }^{1} \mathrm{H}$ NMR spectra (THF- $d_{8}, 298 \mathrm{~K}, 500 \mathrm{MHz}$ ) of PG1 upon irradiation at $365 \mathrm{~nm}$ for different time. 
PG1 $3 \mathrm{~h}$

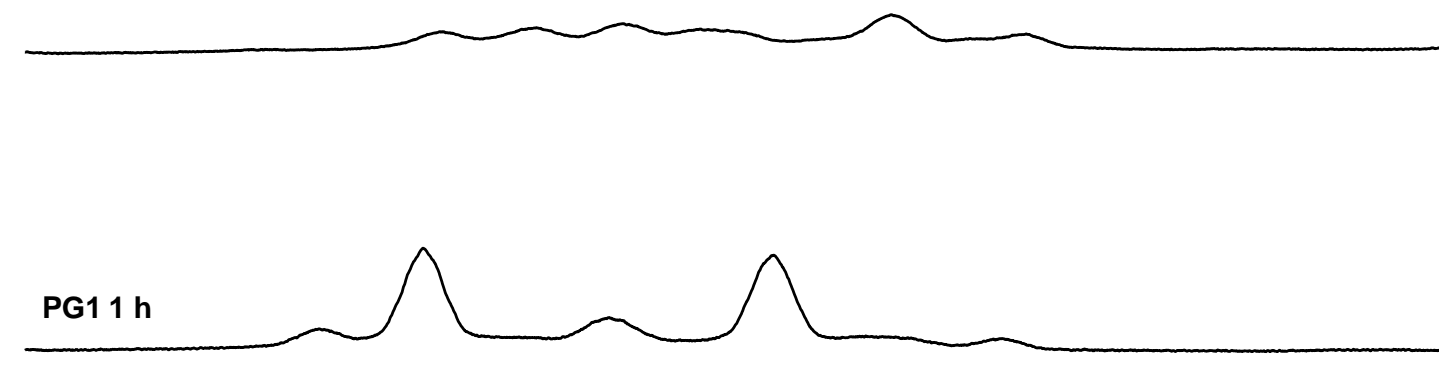

PG1 $0 \mathrm{~h}$

$\begin{array}{lllllllllllllllll}-0.2 & -0.3 & -0.4 & -0.5 & -0.6 & -0.7 & -0.8 & -0.9 & -1.0 & -1.1 & -1.2 & -1.3 & -1.4 & -1.5 & -1.6 & -1.7\end{array}$

Figure S61. Partial ${ }^{1} \mathrm{H}$ NMR spectra (THF-d8, $298 \mathrm{~K}, 500 \mathrm{MHz}$ ) of PG1 upon irradiation at $365 \mathrm{~nm}$ for different time.

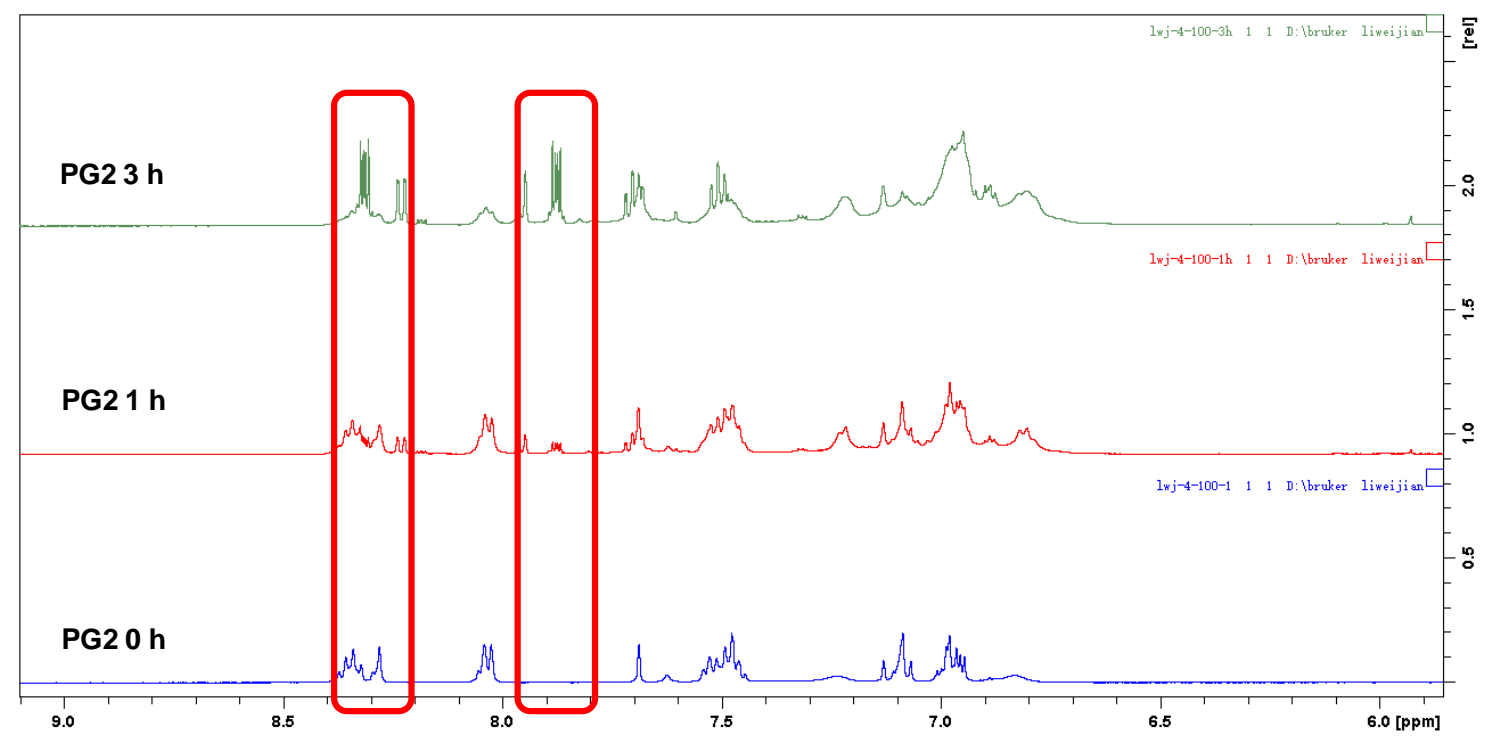

Figure S62. Partial ${ }^{1} \mathrm{H}$ NMR spectra (THF- $d_{8}, 298 \mathrm{~K}, 500 \mathrm{MHz}$ ) of PG2 upon irradiation at $365 \mathrm{~nm}$ for different time. 


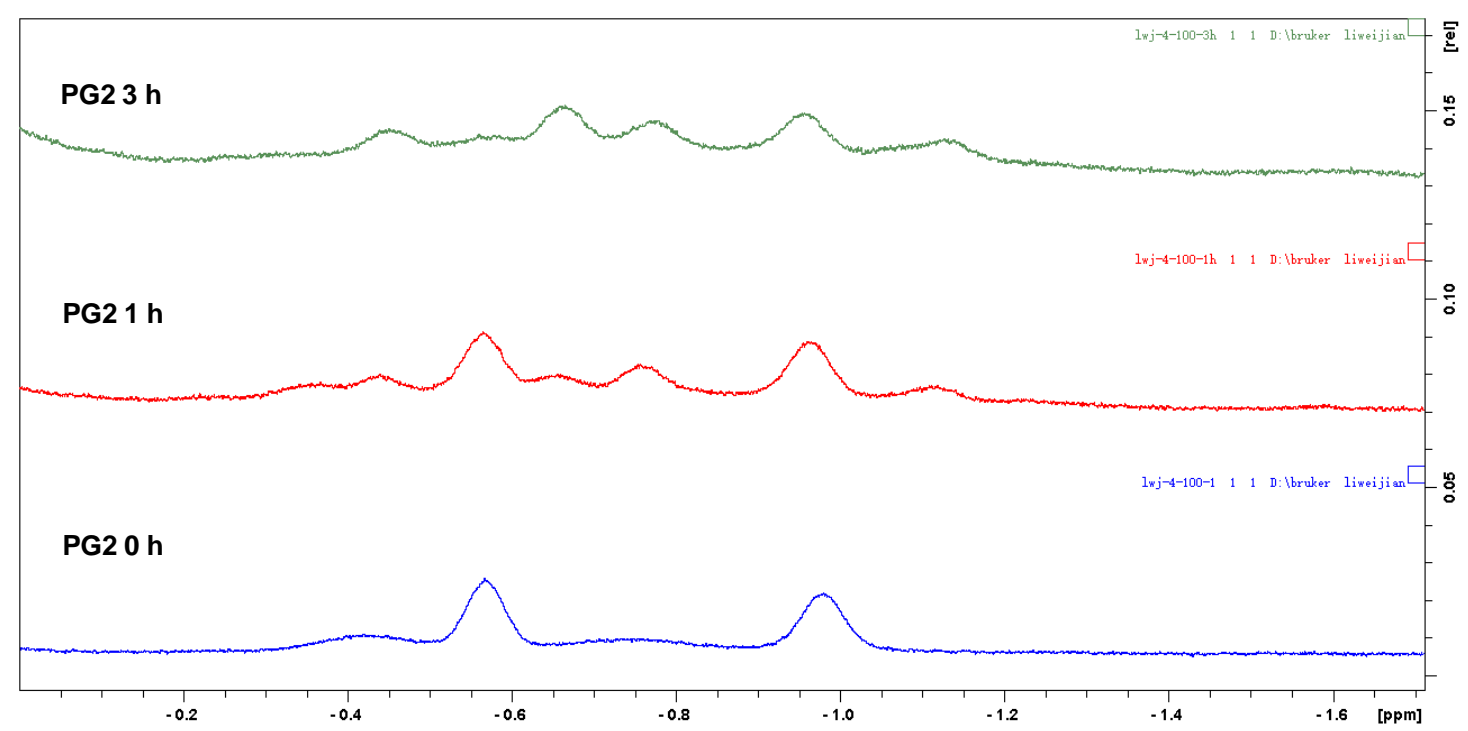

Figure S63. Partial ${ }^{1} \mathrm{H}$ NMR spectra (THF- $d_{8}, 298 \mathrm{~K}, 500 \mathrm{MHz}$ ) of PG2 upon irradiation at $365 \mathrm{~nm}$ for different time. 
Section H. Kinetic analysis on the photolysis of the rotaxane-branched dendrimers

According to the basic principles of chemical kinetics, when the initial concentrations of reactants keep constant, the relationship between reaction rate $(k)$ and half-life $\left(t_{1 / 2}\right)$ follows:

$$
t_{1 / 2} \propto \frac{1}{k} \quad \text { or } \quad t_{1 / 2}=\frac{C}{k}(C=\text { const })
$$

where $t_{1 / 2}$ can be obtained directly from the time-conversion curves. From our experiments, $t_{1 / 2}$ data at were summarized in Table S9.

According to the Arrhenius formula:

$$
k=A \exp \left(-\frac{E_{a}}{R T}\right)
$$

where $E_{a}$ is the apparent activation energy, $A$ is the pre-exponential factor, $R$ is universal gas constant and $T$ is temperature. Thus we can obtain:

$$
\frac{C}{t_{1 / 2}}=A \exp \left(-\frac{E_{a}}{R T}\right)
$$

And then the equation can be further transformed to be:

$$
\ln \left(t_{1 / 2}\right)=\frac{E_{a}}{R} \times \frac{1}{T}+\ln C+\ln A
$$

From the data of half-life $\left(t_{1 / 2}\right)$ at different temperatures $(T)$, we can obtain the apparent activation energy $\left(E_{a}\right)$ of photolysis with the linear fitting method.

Table S9. Half-life $\left(t_{1 / 2}\right)$ of photodecomposition of PGn $(n=1,2,3)$ measured by UVvis spectroscopy in THF solutions with the same concentration of anthracenes $(0.063$ $\mathrm{mM}$ ) at different temperatures.

\begin{tabular}{cccc}
\hline Half life & PG1 & PG2 & PG3 \\
\hline $\mathbf{t}_{\mathbf{1} / \mathbf{2}}$ at $25^{\circ} \mathrm{C}(\mathrm{s})$ & 2187 & 612 & 351 \\
\hline $\mathbf{t}_{\mathbf{1} / \mathbf{2}}$ at $30^{\circ} \mathrm{C}(\mathrm{s})$ & 1450 & 467 & 337 \\
\hline $\mathbf{t}_{\mathbf{1} / \mathbf{2}}$ at $35^{\circ} \mathrm{C}(\mathrm{s})$ & 1181 & 423 & 329 \\
\hline $\mathbf{t}_{\mathbf{1} / \mathbf{2}}$ at $40^{\circ} \mathrm{C}(\mathrm{s})$ & 607 & 270 & 307 \\
\hline $\mathbf{t}_{\mathbf{1} / \mathbf{2}}$ at $45^{\circ} \mathrm{C}(\mathrm{s})$ & 368 & 193 & 294 \\
\hline
\end{tabular}




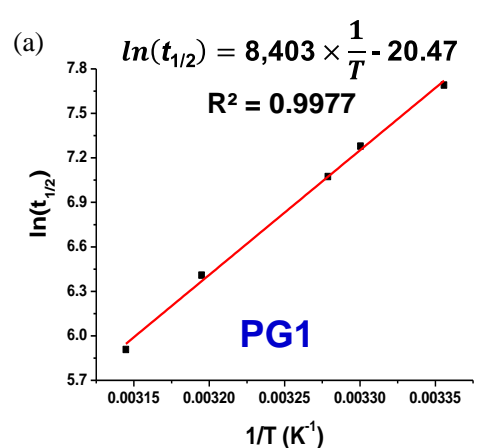

$E_{a}=69.87 \mathrm{~kJ} / \mathrm{mol}$
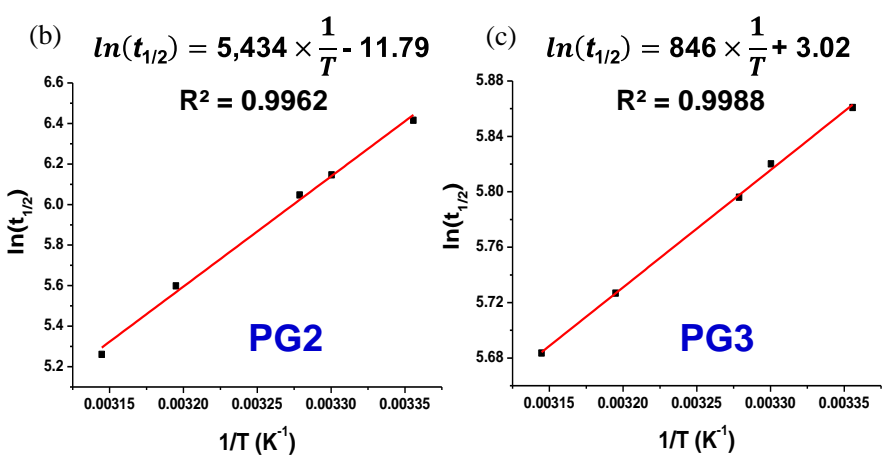

$E_{a}=45.19 \mathrm{~kJ} / \mathrm{mol}$

$E_{a}=7.04 \mathrm{~kJ} / \mathrm{mol}$

Figure S64. Linear fitting of the half-life $\left(t_{1 / 2}\right)$ to the reaction temperature $(T)$ to give the apparent activation energy $\left(E_{\mathrm{a}}\right)$ of photodecomposition of $\mathbf{P G n}(\mathrm{n}=1,2,3)$. 


\section{Section I. Supplementary data}

The additional control experiments were performed, in which the dendrimers PG1, PG2, and PG3 were excited at about $395 \mathrm{~nm}$ to study the singlet oxygen generation efficiency. As shown in Figures S65-67 and Table S10, the normalized ${ }^{1} \mathrm{O}_{2}$ quantum yields of PG1, PG2, and PG3 upon excitation at about $395 \mathrm{~nm}$ were nearly similar with that of $365 \mathrm{~nm}$. The observed results indicated that the third-generation rotaxanebranched dendrimer PG3 showed higher ${ }^{1} \mathrm{O}_{2}$ generation efficiency than that of the firstgeneration rotaxane-branched dendrimer PG1 and second-generation rotaxanebranched dendrimer PG2 upon excitation at both $395 \mathrm{~nm}$ and $365 \mathrm{~nm}$.

(a)

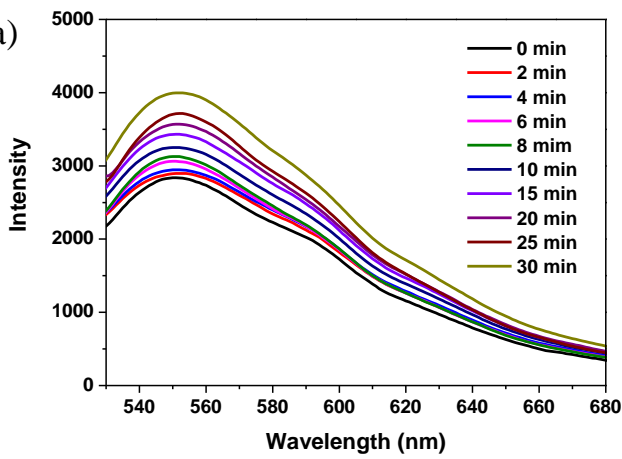

(b)

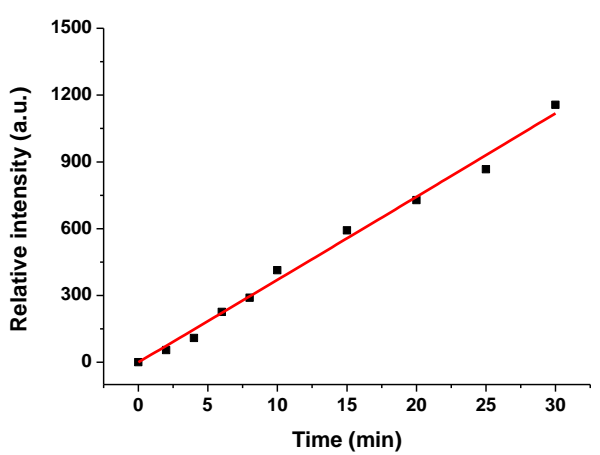

Figure 65. (a) Emission spectrum of SOSG in the presence of PG1 as a function of irradiation time ( $\lambda_{\mathrm{ex}}=504 \mathrm{~nm}, 0.042 \mathrm{mM}$ in THF of anthracene unit). (b) Singlet oxygen sensor green $(10 \mu \mathrm{M})$ relative fluorescence intensity $v s$. time curve. Irradiation light for singlet oxygen generation was at 395-400 nm (SSSTECH-LAL1CV1.0, 12W, 100-240 VAC 50/60 Hz).
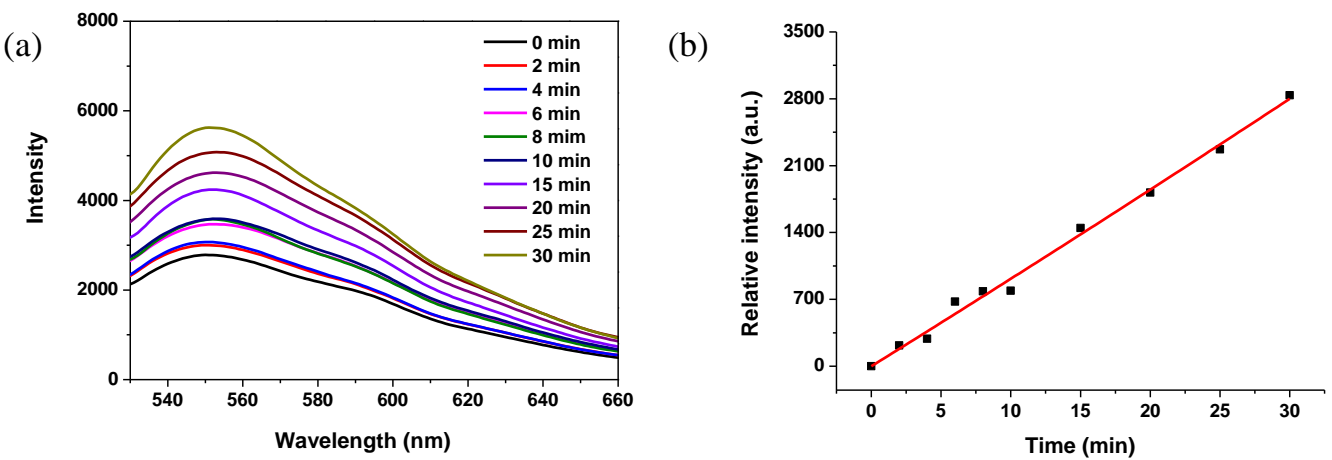

Figure 66. (a) Emission spectrum of SOSG in the presence of PG2 as a function of irradiation time ( $\lambda_{\mathrm{ex}}=504 \mathrm{~nm}, 0.042 \mathrm{mM}$ in THF of anthracene unit). (b) Singlet oxygen sensor green $(10 \mu \mathrm{M})$ relative fluorescence intensity $v s$. time curve. Irradiation light for singlet oxygen generation was at 395-400 nm (SSSTECH-LAL1CV1.0, 12W, 100-240 VAC 50/60 Hz). 

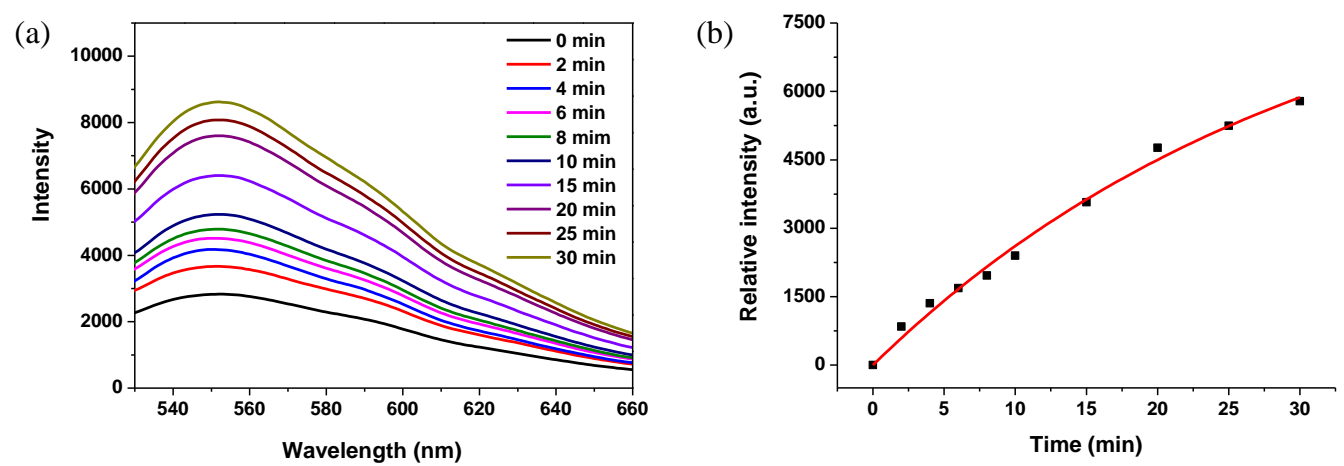

Figure 67. (a) Emission spectrum of SOSG in the presence of PG3 as a function of irradiation time $\left(\lambda_{\mathrm{ex}}=504 \mathrm{~nm}, 0.042 \mathrm{mM}\right.$ in THF of anthracene unit). (b) Singlet oxygen sensor green $(10 \mu \mathrm{M})$ relative fluorescence intensity $v s$. time curve. Irradiation light for singlet oxygen generation was at 395-400 nm (SSSTECH-LAL1CV1.0, 12W, 100-240 VAC 50/60 Hz).

Table S10. Fitting parameters of singlet oxygen generation rate. Irradiation light for singlet oxygen generation was at 395-400 nm (SSSTECH-LAL1CV1.0, 12W, 100-240 VAC $50 / 60 \mathrm{~Hz})$.

\begin{tabular}{llll}
\hline & $\mathrm{R}^{2}$ & $A k$ & Normalized yield \\
\hline PG1 & 0.9909 & $36.9 \pm 4.1$ & 1.0 \\
\hline PG2 & 0.9927 & $90.4 \pm 6.3$ & 2.4 \\
\hline PG3 & 0.9914 & $304.7 \pm 10.9$ & 8.3 \\
\hline
\end{tabular}




\section{Section J. References}

1. Qian X. et al., Chem. Eur. J. 2016, 22, 6881-6890.

2. Wang X.-Q. et al., Nat. Commun. 2018, 9, 3190.

3. Stephens P. J. et al., J. Phys. Chem. 1994, 98, 11623-11627.

4. Grimme S. et al., J. Chem. Phys. 2010, 132, 154104.

5. Grimme S. et al., J. Comput. Chem. 2011, 32, 1456-1465.

6. Ditchfield R. et al., J. Chem. Phys. 1971, 54, 724-728.

7. Hehre W. J. et al., J. Chem. Phys. 1972, 56, 2257-2261.

8. Hariharan P. C. et al., Theor. Chim. Acta 1973, 28, 213-222.

9. Frisch M. J. et al., Gaussian 16 Rev. A.03, Wallingford, CT, 2016.

10. Grimme S. et al., J. Chem. Theory Comput. 2017, 13, 1989-2009.

11. C. Bannwarth et al., J. Chem. Theory Comput. 2019, 15, 1652-1671.

12. Pracht, P. et al., ChemRxiv: 2019.

13. Lin Y.-S. et al., J. Chem. Theory Comput. 2013, 9, 263-272.

14. Weigend F. et al., Phys. Chem. Chem. Phys. 2005, 7, 3297-3305.

15. Andrae D. et al., Theor. Chim. Acta 1990, 77, 123-141.

16. Koseki S. et al., J. Phys. Chem. 1995, 99, 12764-12772.

17. Neese F. et al., J. Comput. Chem. 2003, 24, 1740-1747.

18. Neese F. et al., Chem. Phys. 2009, 356, 98-109.

19. Weigend F. et al., Phys. Chem. Chem. Phys. 2006, 8, 1057-1065.

20. Neese F. et al., WIREs Comput. Mol. Sci. 2012, 2, 73-78.

21. Neese F. et al., WIREs Comput. Mol. Sci. 2018, 8, e1327.

22. Humphrey W. et al., J. Mol. Graphics 1996, 14, 33-38. 